University of Louisville

ThinkIR: The University of Louisville's Institutional Repository

Electronic Theses and Dissertations

$8-2020$

\title{
Incarceration experiences of older African American adults living with HIV - a constructivist grounded theory study.
}

Verena Schmidt

University of Louisville

Follow this and additional works at: https://ir.library.louisville.edu/etd

Part of the Social and Behavioral Sciences Commons

\section{Recommended Citation}

Schmidt, Verena, "Incarceration experiences of older African American adults living with HIV - a constructivist grounded theory study." (2020). Electronic Theses and Dissertations. Paper 3511. https://doi.org/10.18297/etd/3511

This Doctoral Dissertation is brought to you for free and open access by ThinkIR: The University of Louisville's Institutional Repository. It has been accepted for inclusion in Electronic Theses and Dissertations by an authorized administrator of ThinkIR: The University of Louisville's Institutional Repository. This title appears here courtesy of the author, who has retained all other copyrights. For more information, please contact thinkir@louisville.edu. 
INCARCERATION EXPERIENCES OF OLDER AFRICAN AMERICAN ADULTS

LIVING WITH HIV - A CONSTRUCTIVIST GROUNDED THEORY STUDY

\author{
By \\ Verena Schmidt \\ B.A., KSFH Munich, 2010 \\ MSSW., University of Louisville, 2011
}

\begin{abstract}
A Dissertation
Submitted to the Faculty of the

Raymond A. Kent School of Social Work of The University of Louisville

in Partial Fulfillment of the Requirements for the Degree of
\end{abstract}

\author{
Doctor of Philosophy \\ in Social Work
}

Raymond A. Kent School of Social Work

University of Louisville

Louisville, Kentucky

August 2020 
Copyright 2020 by Verena Schmidt

All rights reserved 

INCARCERATION EXPERIENCES OF OLDER AFRICAN AMERICAN ADULTS

LIVING WITH HIV - A CONSTRUCTIVIST GROUNDED THEORY STUDY

By

Verena Schmidt

B.A., KSFH Munich, 2010

MSSW., University of Louisville, 2011

A Dissertation Approved on

August $7^{\text {th }}, 2020$

by the Following Dissertation Committee:

Lesley Harris (Chair)

Bibhuti Sar

Sunshine Rote

Jelani Kerr 


\section{DEDICATION}

For my parents Tom and Bonnie LeCompte

who sacrificed so much to make this possible for me. 


\section{ACKNOWLEDGMENTS}

First and foremost, I want to thank the courageous individuals who participated in this study. I would like to thank the members of my committee Dr. Sar, Dr. Rote and Dr. Kerr for their valuable suggestions, useful comments and continued support throughout this research. Dr. Lesley Harris my dissertation chair and mentor throughout my doctoral studies deserves my deepest gratitude. Without her patience, words of encouragement and invaluable guidance throughout this process this research would not have been possible. I also want to express my appreciation to Dr. Sar and his support during my time at Kent School. My special thanks to the members of the research team of the original study, Dr. Crawford, Dr. Harris and Dr. Kerr, as well as graduate research assistants Nanette Bowman and fellow doctoral candidate Tammi Thomas.

Completing this work would not have been possible without my family. My parents, grandparents, and church family supported me in every way possible. I am grateful for their unconditional love, sacrifices and most of all prayers. Finally, I want to thank God for allowing me to undertake and complete this journey. Praise be to God. 


\section{ABSTRACT \\ INCARCERATION EXPERIENCES OF OLDER AFRICAN AMERICAN ADULTS \\ LIVING WITH HIV \\ - A CONSTRUCTIVIST GROUNDED THEORY STUDY \\ Verena Schmidt}

August $7^{\text {th }}, 2020$

Two epidemics disproportionally impact older (50 years and over) African Americans compared to the general U.S. population: Incarceration and HIV/AIDS. Those aged 50 and older constitute the fastest growing age group of persons who are incarcerated in the United States. It is estimated that by the year 2030 about one third of the incarcerated population in the U.S. will be aged 55 years and older. While areas of "Incarceration, racial disparities and HIV" as well as "Aging and HIV" have been well studied and discussed in the literature, little is known about the lived incarceration experiences of African American persons living with HIV/AIDS (AAPLWHA). Thus, the purpose of this constructivist grounded theory study was to understand the processes older AAPLWHA experience related to their incarceration and engagement in care. The two main goals of this study were: 1) To understand and provide deep description of the varied dimensions of the experience of incarceration among older AAPLWHAs and 2) To develop an inductive theory of the process related to incarceration experiences among older AAPLWHAs and their engagement in care. Questions supporting and guiding these goals included: 1) How do older AAPLWHA draw meaning from their incarceration experiences? 2) How do incarceration experiences and understandings of their meaning relate to the process of engagement in care for older AAPLWHA? The nature of these 
questions warranted a qualitative approach designed to gain an in-depth understanding from older AAPLWHAs and their incarceration experiences. Methods included in-depth interviews with twenty-two older AAPLWHA who had an incarceration history. Additionally, seven participants were selected for in-depth follow-up interviews. The results include an exploration of the influence of HIV and incarceration stigma, different pathways (jail versus prison), and the impact of age and race on older AAPLWHAs incarceration experience. This study investigated the behavioral and psychological processes related to engagement in care for formerly incarcerated older AAPLWHA resulting in the formulation of an inductive theory titled: "Older AAPLWHAs Journey Towards Engagement in Care during Incarceration."

The inductive theory explains how older AAPLWHAs engaged in the process related to their HIV care from the point of entering the correctional system to in-care experiences and of linkage to care post-release. In the context of program and policy development, the findings of this study can provide the following insights: 1) Interventions within correctional settings that prioritize HIV care and decrease HIV related stigma 2) Greater access to long-term services and linkage to care post-release, and 3) Collaboration of AIDS Service Organizations (ASOs), correctional facilities and health care providers to guide improvements of the HIV care cascade for older incarcerated AAPLWHAs and post-incarceration continuity of care. 


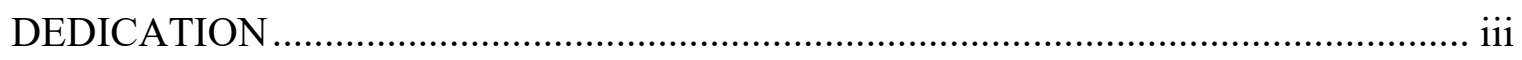

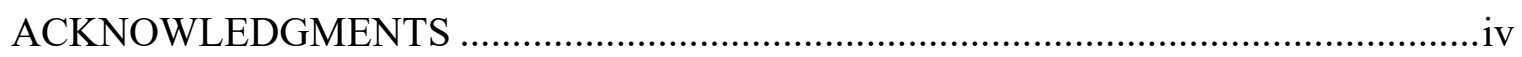

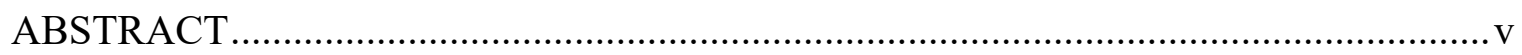

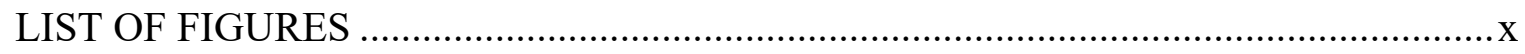

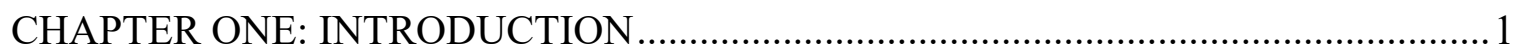

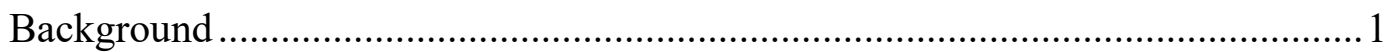

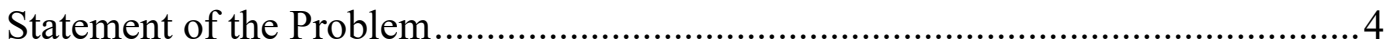

Purpose of the Study and Research Questions ................................................5

Study Overview and Research Approach ….....................................................

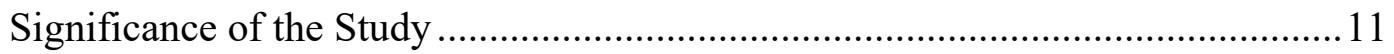

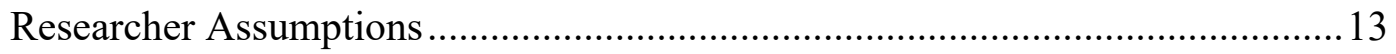

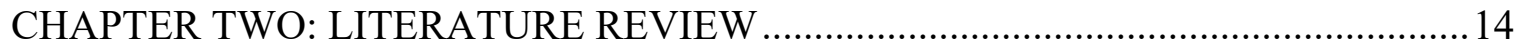

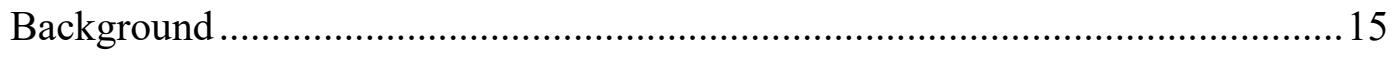

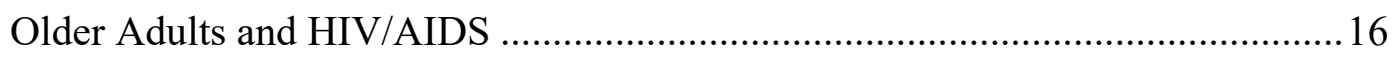

Older African American Adults and HIV ........................................................ 17

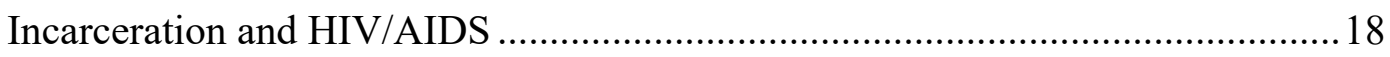

African Americans and Incarceration ............................................................22

Incarceration and Older Adults ..................................................................2 22

Intersection of Incarceration, Health Care and HIV …......................................22

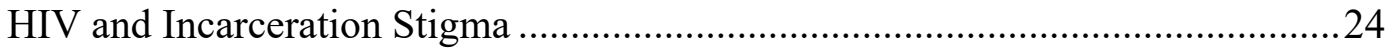

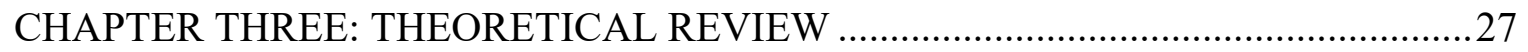

The Comprehensive Health Seeking and Coping Paradigm...............................28 
Older AAPLWHA with Incarceration Experiences and the CHSCP

Incarceration Experiences of Older AAPLWHA: An Emerging Conceptual

Framework

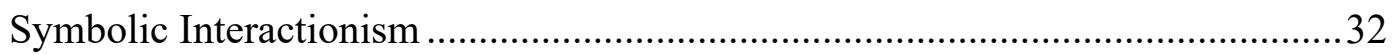

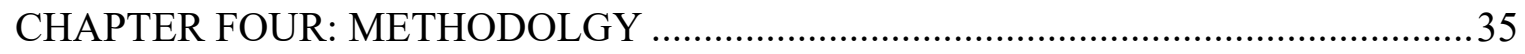

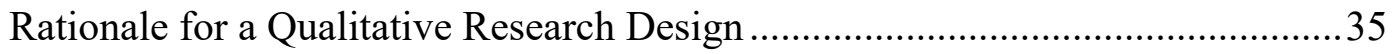

Social Constructivism and Rationale for Constructivist Grounded Theory

Methodology .36

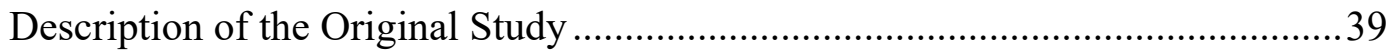

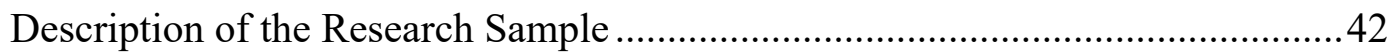

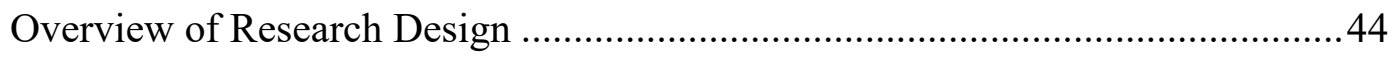

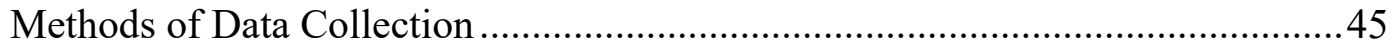

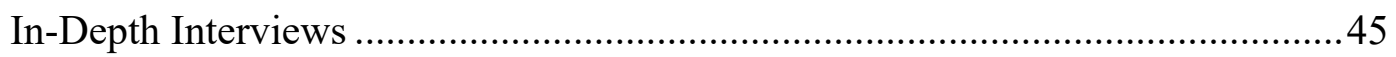

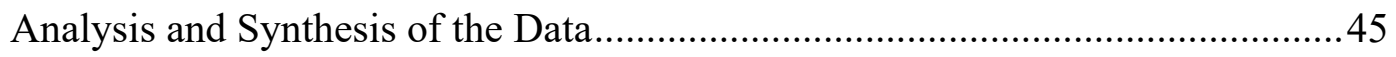

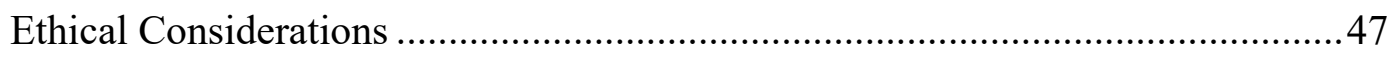

Rigor, Trustworthiness, Positionality \& Reflexivity Statement .........................47

CHAPTER FIVE: PARTICIPANT DEMOGRAPHICS AND OVERVIEW OF THE PROCESS OLDER AAPLWHA EXPERIENCE RELATED TO THEIR

INCARCERATION AND ENGAGEMENT IN CARE..................................................50

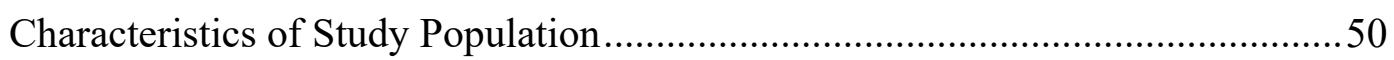

Overview of the Process Older AAPLWHA Experience Related to Their Incarceration and Engagement in Care ..............................................................53

CHAPTER SIX: HOW DO OLDER AAPLWHA DRAW MEANING FROM THEIR

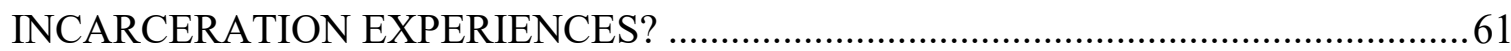

Sub-Question: Explore the Influence of HIV Stigma ......................................62

Sub-Question: Explore the Influence of Incarceration Stigma ............................69

Sub-Question: Explore the Different Pathways through which the Incarceration was Experienced

Sub-Question: Explore How Age and Race Impacted One's Incarceration Experience...... 
CHAPTER SEVEN: HOW DO INCARCERATION EXPERIENCES AND

UNDERSTANDINGS OF THEIR MEANING RELATE TO ENGAGEMENT IN

CARE?

Understanding the Behavioral Processes ....................................................... 83

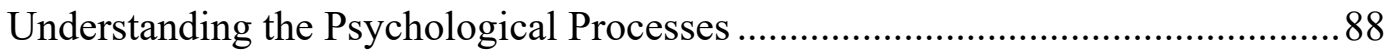

CHAPTER EIGHT: AN INDUCTIVE THEORY OF THE PROCESS RELATED TO THE INCARCERATION EXPERIENCES AMONG OLDER AAPLWHA AND THEIR

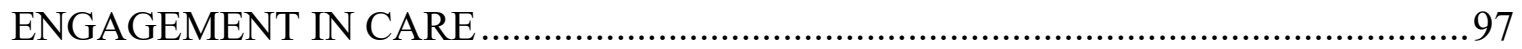

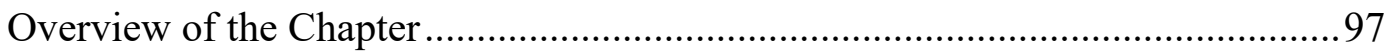

Overview of the Inductive Theory "Older AAPLWHAs Journey towards Engagement in Care during Incarceration" .98

CHAPTER NINE: DISCUSSION 107

How do Older AAPLWHA Draw Meaning from Their Incarceration Experiences?

How do Incarceration Experiences and Understandings of Their Meaning Relate to the Process of Engagement in Care?

Limitations and Suggestions for Future Research 117

Conclusion 118

REFERENCES

APPENDICES

Appendix A

Appendix B

Appendix C

Appendix D 


\section{LIST OF FIGURES}

FIGURE

PAGE

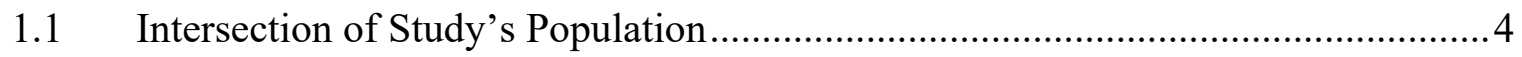

3.1 Incarceration Experiences of older AAPLWHA an Emerging Conceptual

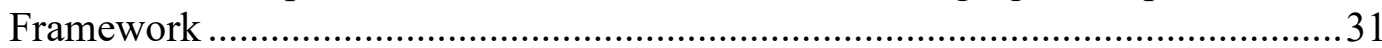

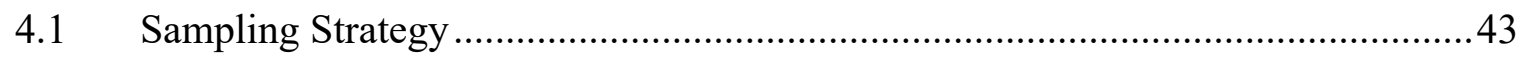

5.1 Overview of the Process older AAPLWHA experience related to their Incarceration and Engagement in Care ............................................................55

6.1 Experiencing Internal and/or External HIV Stigma........................................62

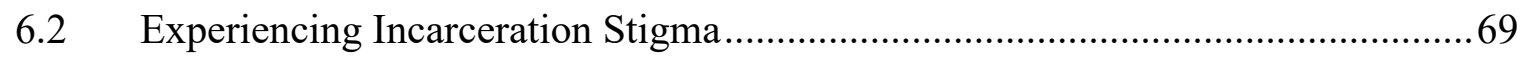

6.3 Pathways through which the Incarceration was experienced ..............................74

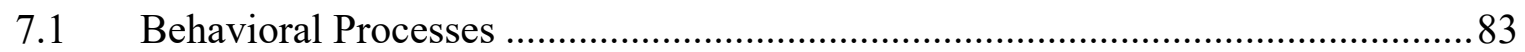

7.2 Psychological Processes related to Engagement in Care for formerly incarcerated older AAPLWHA

8.1 Model Representation of the Inductive Theory depicting the Phases and the

9.1. The HIV Treatment Cascade from HIV.gov 116 


\section{CHAPTER ONE: INTRODUCTION}

This chapter begins with an overview of the context and background that frames this study on the incarceration experiences of older African American adults living with HIV. Following this is the problem statement, the purpose of the study, the research questions and the research approach. Next this chapter highlights the significance of the study, which is to provide much needed insight into the incarceration experiences of older African American adults living with HIV. The chapter concludes with the researcher's assumptions about the situation and context in which the research takes place.

\section{Background}

Two epidemics disproportionally impact older (50 years and over) African Americans compared to the general U.S. population: incarceration and HIV/AIDS. In 2018, African Americans made up 2.5 million or $33 \%$ of the total 2.3 million incarcerated individuals (Minton \& Golinelli, 2014; U.S. Census Bureau, 2018; Sawyer \& Wagner, 2019). African American men make up 36 percent of the U.S. population of men in jail and 37 percent of the U.S. population of men in prison (Carson, 2014; Mayer, Spaulding \& Stephenson et al., 2002; U.S. Census Bureau, 2014). Additionally, African American women are twice as likely to be incarcerated when compared to Caucasian women (Minton \& Golinelli, 2014). Even though the prevalence of the human immunodeficiency virus (HIV) in prisons has decreased since the late 1990s, mass incarceration has contributed to the ongoing HIV epidemic within correctional settings. An estimated $1.5 \%$ of all inmates in U.S. state and federal prisons are HIV positive (Fazel \& Baillargeon, 2011). Not only is the HIV rate among incarcerated individuals 5 to 7 
times higher than that of the general U.S. population, but the number of confirmed AIDS cases in incarceration settings is also 2.5 times higher than the general population (AIDSinfonet, 2014; Iroh, Maya \& Nijhawan et al., 2015). Although HIV/AIDS affects all ethnicities, the HIV rates are highest among incarcerated African Americans (CDC, 2018; Brinkley-Rubinstein \& Turner, 2013).

In 2018, African Americans accounted for 41\% of all new HIV infections, but represented only $13 \%$ of the U.S. population (CDC, 2018). Similarly, African Americans made up 47 percent of new AIDS diagnoses (CDC, 2018). Across ethnicities, individuals aged 50 and older are the fastest growing segment of people living with HIV in the United States. Major advances in HIV treatment (i.e. effective medications) since the outbreak of the HIV epidemic in the U.S. have resulted in individuals not only living longer, but also aging with the disease (CDC, 2018; High, Brennan-Ing \& Clifford et al., 2012; Justice, 2010; United States Census Bureau, 2018). Therefore, aging with HIV not only creates a new set of age-related challenges, but also constitutes a relatively new phenomenon of people growing older with HIV. In 2017, the HIV rate among 50-54 year-old African Americans was 6.8 times higher than that of comparably aged Caucasians, suggesting that older African American adults are more prone to HIV/AIDS (acquired human immunodeficiency virus) infection than their racial counterparts (CDC, 2018). The disproportionate rate of HIV in older African Americans, combined with the paucity of research on this population has created a gap in knowledge in terms of how older African Americans experience aging with HIV/AIDS.

With regard to older adults and incarceration, individuals aged 50 and older constitute the fastest growing age group of incarcerated persons in the United States. The American Civil Liberties Union (ACLU) (2012) reported that 124,900 incarcerated persons are aged 55 and older compared to 8,853 prisoners in 1981. It is estimated that 
by the year 2030, about one third (approximately 400,000) of the incarcerated population in the United States will be 55 years of age and older (ACLU, 2012; Rich et al., 2013). The literature has documented that biological aging for persons who are incarcerated occurs more rapidly than other individuals in their age group (ACLU, 2012; Aday \& Krabil, 2012; Beckwith et al., 2010). Falter (2006) describes that on average, incarcerated individuals are physiologically 10 to 15 years older compared to their nonincarcerated counterparts. Although research on the health of older incarcerated adults remains scarce, existing literature states that a history of incarceration is associated with higher rates of chronic and infectious diseases, mental and physical health conditions, and increased mortality when compared to individuals without incarceration histories. An accelerated aging process cannot be explained by one factor, but is related to an array of determinants, such as being in a confined environment, stress, poor diet, drug and/ or alcohol abuse, poor health and health care, low educational attainment and socioeconomic status (Aday, 2003; Falter, 2006; Hayes, Burns, Turnbull, \& Shaw 2012; Spaulding et al., 2011). Given the reality accelerated aging, substandard medical care, environmental and social constraints in the correctional system, understanding the experiences of particular subgroups is warranted. This study is premised on the problem that incarceration experiences are of concern for older AAPLWHA.

Considering that older adults $(50+)$, African Americans, and incarcerated individuals are each vulnerable and marginalized populations, the intersection of the three can create a complexity of challenges that are exacerbated when crossover occurs. The figure below visualizes the intersection of this study's population. 


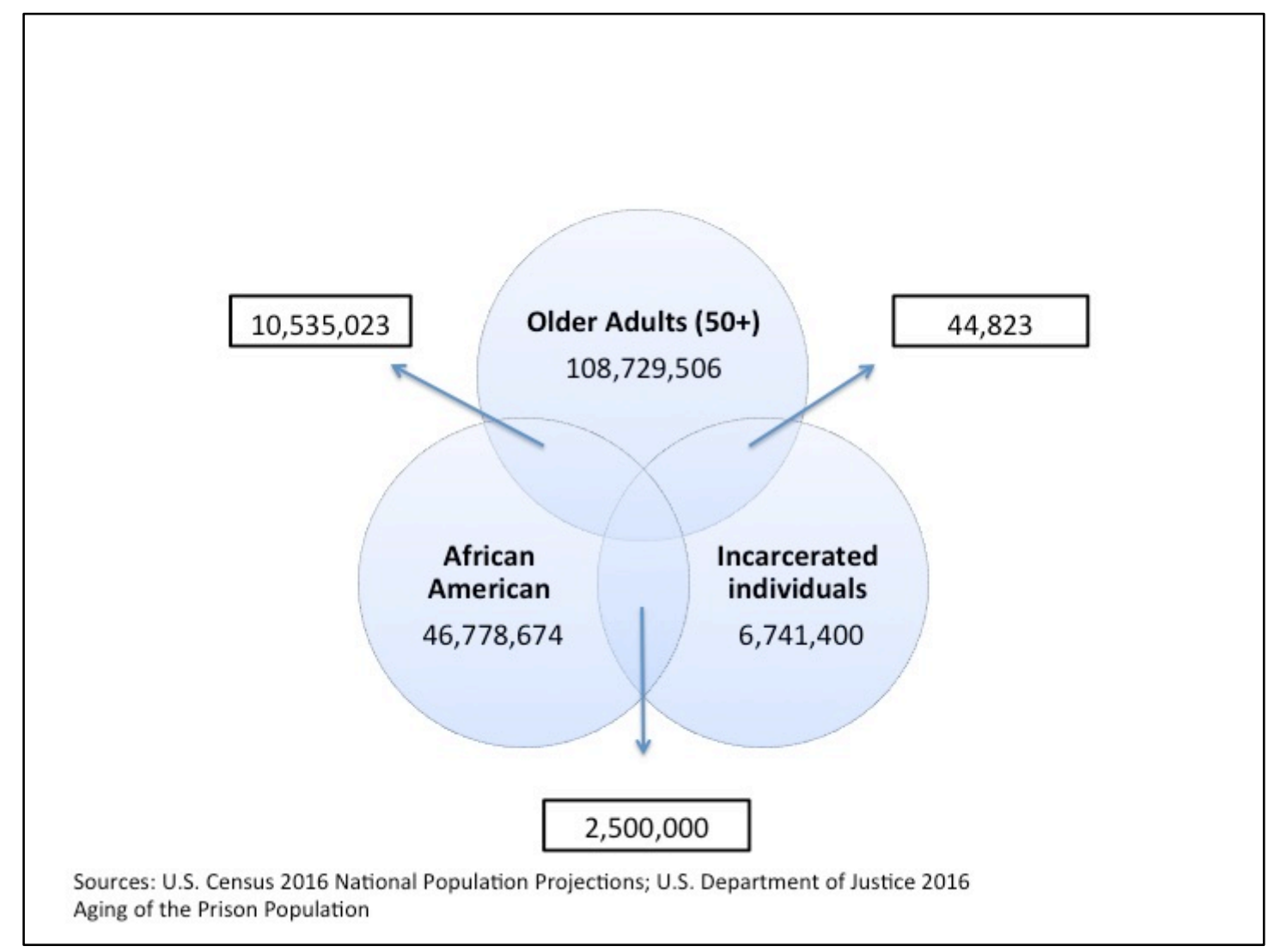

Figure 1.1 Intersection of Study's Population

\section{Statement of the Problem}

Experiencing an "accelerated" or "accentuated" aging process is not unique to older incarcerated individuals; Individuals living with HIV/AIDS also age at a disproportionally higher rate than their healthy counterparts (Martin \& Volberding, 2010; Pathai, Bajillian \& Landay et al., 2013). Hence, incarcerated PLWHA who are 50 years and older can experience accelerated biological aging not only as a result of living with HIV, but also due to their incarceration history. This puts older incarcerated PLWHA at increased risk to experience age- related multiple illnesses (e.g., dementia) as well as HIV-associated conditions such a hepatitis B, hepatitis C and liver disease. Such illnesses require medical attention and can complicate the treatment of HIV (Brothers, Kirkland \& Guaraldi et al., 2014; Rich et al., 2013). HIV/AIDS-related deaths among individuals incarcerated in state prisons are the highest among African Americans and individuals' 
aged 50 and above. In 2010, 43 African American inmates died of AIDS-related death/or complications due to AIDS compared to 23 Caucasian and five Hispanic inmates. Of the 43 deaths of African American inmates, 38 individuals were aged 50 and older (U. S. Department of Justice, 2015).

The incarceration setting presents an array of challenges for older AAPLWHA (i.e. a lack of HIV testing, HIV stigma), but also provides opportunities for prevention, initial diagnosis and treatment (CDC, 2016; Iroh, Maya \& Nihawan, 2015; RowellCunsolo, El-Bassel, \& Hart 2016). Currently, HIV prevalence and incidence is documented in only 19 states. Consequently, available data drastically underestimate HIV rates (CDC, 2017). The state of Kentucky does not routinely test inmates for HIV in any correctional setting including prisons or jails. At the present time, only a basic health screening is mandated for all individuals entering the correctional system (Commonwealth of Kentucky DOC, 2018; Pope, 2009). In addition, Kentucky’s correctional system neither tests inmates for HIV upon entry nor prior to release in the community (Center for HIV Law and Policy, 2017; Kentucky Department of Corrections, 2017). To date, no theory addresses the incarceration experiences of older AAPLWHA. Considering the number of older AAPLWHA, and the fact this population is disproportionally affected by HIV and incarceration, warrants the need for a study to generate findings that discover the processes related to older AAPWLHAs incarceration experiences and engagement in care.

\section{Purpose of the Study and Research Questions}

This study emerged from a larger study (referred to as the original study) titled: “African American Older Adults Living with HIV: Exploring Stress, Stigma, and Engagement in HIV Care.” Over $60 \%$ of participants of the original study discussed past incarceration experiences during their in-depth interviews, and how these experiences 
impacted their engagement in HIV care. This unexpected finding led to the emergence of the current study.

The purpose of this constructivist grounded theory study was to understand the processes older African American adults experience related to their incarceration and engagement in care. At this stage in the research, incarceration experiences encompassed HIV testing, disclosure experiences and engagement in care. The broad approach in defining incarceration experiences encouraged adaption and flexibility for exploring and responding to the data as supported by the constant comparative approach and iterative nature of a grounded theory studies (Charmaz, 2014).

There were two primary goals of this study:

1. To understand and provide deep description of the varied dimensions of the experience of incarceration among older AAPLWHAs

2. To develop an inductive theory of the process related to incarceration experiences among older AAPLWHAs and their engagement in care The study had the following research questions with embedded aims:

1. How do older AAPLWHA draw meaning from their incarceration experiences?

a. Explore the influence of stigma (HIV and incarceration-related stigma).

b. Explore the different pathways (i.e. jail versus prison) through which incarceration is experienced.

c. How do age and race impact one's incarceration experience?

2. How do incarceration experiences and understandings of their meaning relate to the process of engagement in care for older AAPLWHA? 
a. Understand the behavioral and psychological processes related to engagement in care for formerly incarcerated older AAPLWHA.

\section{Study Overview and Research Approach}

This constructivist grounded theory (CGT) study sought to understand the processes experienced by older African American adults related to their incarceration experiences and their engagement in care. The study aligns with a constructivist epistemology of subjectivism and acknowledges the researcher's involvement in the meaning making process (Wertz, Charmaz, McMullen \& Josselson et al., 2011). The Comprehensive Health Seeking and Coping Paradigm (CHSCP) (Nyamathi, 1989), along with the literature on aging, HIV and incarceration, informed this research. The CHSCP (Nyamathi, 1989) framework has been used to describe future care engagement of African Americans with rapid HIV testing (Dalmida, Mc Dougal, Mugoya, Foster et al., 2016). Additionally, the framework identified factors relating to HIV risk level among homeless African American women (Nyamathi, 1992), and identified health-seeking challenges among specific populations (Hudson, Nyamathi, Greengold, Slagle et al., 2010; Harris, Crawford, Kerr, Thomas et al., 2020). The CHSCP is a multidimensional framework and acknowledges HIV and incarceration among African Americans as “syndemic connections” (McCarthy, Myers, Reevers \& Zack, 2016), meaning that more than one disease or epidemic is affecting this population. McCarthy and colleagues (2016) propose that the two epidemics - Incarceration and HIV among African Americans

- are a result of several interrelated conditions, including racial disparities, structural barriers, socioeconomic characteristics, access to resources, sentencing and over policing of predominantly African American neighborhoods (Baumer, 2013; Homer and Milstein 2002; Singer \& Clair 2003; Weitzer, 2017). The CHSCP framework was viewed as a sensitizing concept through which to consider the data and informed the beginning 
research questions. The framework posits that socio-demographic factors, situational factors, personal factors and available resources affect an individual's health- seeking and coping experience. Such factors relate directly to an individual's incarceration experience and his/her engagement in care. I created a tentative emerging conceptual framework, which outlines the major concepts of the CHSCP, incorporates factors specific to older AAPLWHA and addresses the HIV stigma framework (Earnshaw \& Chaudoir, 2009; Earnshaw, Smith, Chaudoir, Amico et al., 2013; Parker and Aggleton, 2003).

CGT is underpinned by the principles of Symbolic Interactionism (SI) and highlights the significance of understanding a situation from an individual point of view (Charmaz, 2014; Glaser \& Strauss, 1967; Strauss \& Corbin 1998). The CGT approach attempts to understand how individuals "construct their realities" and focuses on “interpretative understanding of subjects' meanings" (Charmaz, Denzin \& Lincoln, 2003, p. 250). This study, as consistent with CGT, aimed to gain a deeper understanding of the incarceration experiences of older African American adults living with HIV.

The sample for this study was selected from the original study ("African American older Adults Living with HIV: Exploring Stress, Stigma, and Engagement in HIV Care"), which examined stress, stigma and engagement in the care of older AAPLWHA. Of the original study's 35 participants, 22 individuals discussed incarceration histories during the initial qualitative in-depth interviews exploring stress, stigma and engagement in care.

For the purpose of this study, incarcerated individuals are persons who were confined in a prison or jail, or incarcerated overnight in halfway houses, weekend programs, and similar facilities. To be eligible for this study, participants needed to meet the following criteria: Identify as Black or African American, be 50 years of age or older, 
have a confirmed diagnosis of HIV, have an incarceration history, be currently seeking HIV care, speak and understand English, and provide consent to the interview.

This study was a deeper investigation of the 22 participants who had incarceration histories. Theoretical sampling was employed and seven participants were purposively selected for additional in-depth qualitative interviews to more thoroughly explore their incarceration experiences. The information obtained through the seven in-depth qualitative interviews subsequently formed the basis for the findings of this study as well as the 22 interviews of the original study in which participants discussed their incarceration experiences. Three out of those seven purposively selected participants also participated in a member-checking focus group. All interviews were conducted between May and December 2016 in private offices at the 550 Clinic, the University of Louisville, or in a private location of the participants' choice, including their own homes. Consent was obtained prior to beginning each interview, and all participants received a $\$ 20$ gift card upon completion of the interview.

As consistent with constructivist grounded theory, interviews were viewed as "emergent interactions in which social bonds may develop (Charmaz, 2014 p.91)." According to this understanding, the interviews followed a semi-structured interview guide that allowed for exploration, emergent understandings, validation of experiences, and an understanding of mutuality that is built during the interview between researcher and participant (Charmaz, 2014). The interview guide is provided in Appendix B. All interviews were tape recorded, transcribed verbatim and lasted between 60 to 90 minutes.

The qualitative data analysis was conducted using CGT techniques. Initial line-by line coding of transcripts identified actions and categorized concepts that emerged from the data (Charmaz, 2014). The use of gerunds during the line-by line coding process emphasized a language of action rather than one of themes and topics in the data. Line- 
by line coding was completed for seven of the $29(32 \%)$ transcripts. This process led to the development of focused codes (utilizing most significant and/or frequent codes) aimed to synthesize, sort and conceptualize larger amounts of data, as well as direct the analysis by highlighting what appeared to be important in the emergent analysis. Further, applying focused codes advanced the theoretical direction of the analysis and led to unexpected conceptualizations of phenomena, processes, actions and strategies shared by participants (Charmaz, 2014; Creswell \& Poth, 2017). The constant comparison method, (Corbin and Strauss, 2008) along with open and focused coding, led to analytic categories. I used peer debriefing and consensus building at each stage in the coding process, to develop a codebook consisting of code families with definitions. The 22 transcripts of the original study as well as the 7 transcripts of the additional in-depth interviews and the codebook (consisting of code families with definitions) were transferred onto Dedoose software as an organizational tool for data analysis. Additionally, analytical techniques of situational analysis (situational, relational and positional maps) complement the CGT approach (Clarke, 2003, 2005, 2009). Peer debriefing and consensus building (Erlandson, 1993) occurred at each stage of the coding process. After the codebook was established, I coded the 22 original as well as the seven additional interviews and engaged in peer debriefing after all transcripts were coded. This iterative process helped to identify segments in which codes were not in agreement, so discrepancies could be addressed and code segments edited. The coding and data analysis process continued until saturation was reached or no new information was discovered (Corbin et al., 2008). Throughout the analysis, memo writing was used to document my own reflections and uncover inter-connected patterns within the data in order to explain and reveal the social processes older AAPLWHA engage in (Creswell et al., 2017). 


\section{Significance of the Study}

While the topics of incarceration, racial disparities, and HIV, as well as aging and HIV, have been well studied and discussed in the literature little is known about the lived incarceration experiences of older HIV positive African Americans. There exists a substantial body of literature on older adults and HIV (Capeau, 2011; CDC, 2014; Emlet, 2006, 2006a; Frederiksen-Goldsen, Kim \& Emlet et al., 2011; Gidhei, Simone \& Salow et al., 2013; Heckman, Heckman \& Kochman et al., 2002; Heckman \& Halkitis, 2014; High, et al., 2012; Laurencin, Murdock, Laurencin \& Christensen, 2018; Shippy \& Karpiak, 2005; Justice, 2010) as well as regarding "racial disparities, incarceration and HIV prevalence" (Beckwith et al., 2010; Harris et al., 2020; Johnson, \& Raphael 2009; Rosen, Schoenbach, Wohl, White et al., 2009; Linley, Prejean \& An et al., 2012; Khan, Golin, Friedman, Scheidell et al., 2015; Mauer \& King, 2007; U. S. Department of Justice, 2015). Much of the literature on racial disparities, incarceration, and HIV has focused on diagnosis, treatment and prevention of HIV in the criminal justice system (Spaulding, et al., 2002; Maruschak, 2009; Small; Wood \& Betterridghe at al., 2009; Beckwith et al., 2010). Those studies identified a lack of linkage to care after release, since many former incarcerated individuals often lack resources and access to community health services (Beckwith et al., 2010; Khan et al., 2015; Linley et al., 2012; Springer, Pesant, Hodges, Macura et al., 2004).

The majority of existing studies examining the lived experiences of African American incarcerated PLWHA focuses on the experiences of younger individuals (Brinkley-Rubenstein et al., 2013; Sprague et al., 2016). The incarceration experiences of older African American adults in the context of HIV/AIDS create a different set of challenges than their younger racial counterparts. For example, older African American adults may experience comorbid health issues related to aging (Pathai et al., 2013). 
Emerging literature focuses on the aging experiences of African Americans with HIV (Warren-Jeanpiere, Dillaway, Hamilton, Young et al., 2014) and recognizes the value in exploring long-term survivorship and focuses on individuals who contract the disease at an older age (50 and older) (Brennan, Emlet \& Eady 2011); Emlet, Frederiksen-Goldsen \& Kim; 2013; Emlet, 2014). This study can add to the existing literature about incarceration, older African Americans and HIV and provide unique insights into the processes older AAPLWHA are engaging in.

Understanding the incarceration experiences of older African American adults living with HIV can be useful for AIDS Service Organizations (ASOs), social workers, health care providers and correctional facilities. Addressing disclosure experiences and engagement in care has the potential to create interventions for older AAPLWHA who are or have been, incarcerated. In the context of program and policy development, the findings of this study can provide evidence and insights regarding the needs of this underserved and understudied population. There is a pressing need to understand and address the lived incarceration experiences of older AAPLWHA in a variety of settings (i.e. post release, re-incarceration, HIV education HIV care). Nationally, there is increased awareness of the epidemics of incarceration and HIV, as they disproportionally impact African Americans. To date, no programs exclusively attend to the unique experiences of older AAPLWHA with incarceration histories.

HIV stigma is prevalent in incarceration settings. Zawitz and colleagues (2014) noted that incarcerated PLWHA fear HIV stigma more than disease progression or even death. HIV stigma in correctional facilities is exasperated by the closed-in environment and the lack of privacy, which can lead to isolation, discrimination and an increased vulnerability for physical attacks (Andrinopoulos, Kerrigan \& Figueroa et al., 2010; Juergens, Nowak \& Day, 2011). Insights into the incarceration experiences (i.e. 
disclosure, discrimination) of older AAPLWHA in a variety of settings and contexts (i.e. post release, re-incarceration, HIV care) may aid in the development of practical interventions that address the needs of older AAPLWHA. This study can offer new insights and contribute to the theoretical understanding of the incarceration experiences of older AAPLWHA. Findings from this study suggest future research efforts regarding program design and the development of practical interventions.

\section{Researcher Assumptions}

I aligned myself with a social constructivist epistemology and recognized my role as researcher in knowledge construction. Hence, I referred to myself in the first person at appropriate points throughout this study. I acknowledge that my identity as a young Caucasian female and a German native with an accent who speaks English as a second language, contributed to the way research participants perceived and interacted with me. The fact that I come from another country, with a distinct culture, health care system, and criminal justice system challenged some of my background assumptions in terms of what it means to be incarcerated and living with HIV. There is a potential for my foreign status to have put participants at ease and shift some of the underlying power dynamics. However, it is unclear if maintaining a similar social group, such as the same race, age, gender, or nationality could have influenced the interactions differently (Dwyer \& Buckle, 2009; Kerstetter, 2012). To minimize my own biases during data collection and data analysis, I utilized the research team of the original study (three African American researchers and three Caucasian researchers) as well as the community organizations (such as the House of Ruth, and the Kentucky Care Coordination Program) to discuss findings and my own perceptions (Fernald \& Duclos, 2005; Thomas, Blacksmith \& Reno, 2000). 


\section{CHAPTER TWO: LITERATURE REVIEW}

In order gain a deeper understanding of the incarceration experiences of older AAPLWHA, a scoping review of the literature will be utilized to explore the extent to which these experiences are evidenced within the literature (Rumrill, Fitzgerald \& Merchant, 2010; Thomas, Menon, Boruff, Rodriguez et al., 2014). This approach is consistent with the iterative nature of the qualitative research process and enables the researcher to gain an overview of the nature of the evidence and develop awareness of concepts that relate to the substantive area (Hesse-Biber, 2010). A scoping review helps researchers identify broad themes within the literature, locate knowledge gaps and prevents the researcher's thinking from being overly influenced by pre-existing frameworks and models that may lead to bias. As new discoveries emerged during the research process and data analysis, the literature review was revised to provide context in which to understand the findings (Arksey \& O’Malley, 2005; Hesse-Biber, 2010). The two main research questions guided this literature review: 1) How do older AAPLWHA draw meaning from their incarceration experiences? 2) How do incarceration experiences and understandings of their meaning relate to the process of engagement in care for older AAPLWHA?

To conduct this literature review, multiple information sources were searched including books, dissertations, Cochrane Database of Systematic Reviews, MEDLINE, Google Scholar, EBSCO, Sage, PubMed, the American Journal of Public Health and CDC websites. These peer-reviewed, evidence-based primary studies were used to review the literature and gain an overview of the substantive area in the relevant 
literature. Key words and specific search terms used included the following: HIV and Incarceration, HIV in Kentucky, HIV demographics, HIV and perceptions, HIV and poverty, HIV and homelessness, HIV-associated health risk behaviors, HIV/AIDS-related stigma, incarceration stigma and HIV, SDH and HIV beliefs, HIV care in incarceration settings, race and HIV, and older adults and HIV.

This chapter will first explain the background and context of the two epidemics: incarceration and HIV among African American older adults in the U.S. Next, the chapter will provide an overview of what is known about older AAPLWHA and their incarceration experiences. Finally, this chapter will describe the context of the epidemics, noting key cultural and environmental factors, such as the HIV stigma that is attached to HIV/AIDS in the older AA community.

\section{Background}

It has been thirty years since the outbreak of the HIV/AIDS epidemic in the U.S. The diagnosis of being HIV positive has shifted from a fatal condition to that of a chronic and more acute disease. During the late 1980s, medications such as Azidothymidine (AZT) were introduced and slowed the progression of the HIV virus. However, in order for the medications to work properly, individuals had to take as many as 24 pills per day for the rest of their lives and deal with an array of medications' side effects. As a result of improved treatment the number of HIV related deaths in the U.S. decreased from 50,000 to 6,721 between 1997 and 2014 (CDC, 2014). This reduction was largely due to the development of highly effective antiretroviral therapy (HAART) in 1996. The HAART medication regimen consists of two to three different drug combinations, which, if taken daily, halt the virus' growth in the body, control viral load, and delay or prevent the onset of symptoms or progression to AIDS. Besides scientific advancements such as HAART, more recent development of Pre-exposure prophylaxis (PrEP) allows 
individuals to live longer and age with the disease. While acknowledging the major strides against the HIV epidemic, HIV continues to manifest among subpopulations such as gay and bisexual men and women, minorities (i.e. African Americans), incarcerated populations and injection drug users (CDC, 2018).

\section{Older Adults and HIV/AIDS}

The HIV disease has been increasingly recognized as a phenomenon impacting older adults, both in the United States and worldwide (CDC, 2018; High et al., 2012; Justice, 2010). Individuals aged 50 and older are the fastest growing segment of PLWHA in the U.S. Aging with HIV not only creates a new set of age-related challenges (i.e. geriatric symptoms, prevalence of comorbidities), but also requires social service and medical professionals to attend to a growing population of HIV positive older adults. In 2016, nearly 50 percent of the PLWHA were aged 50 years and older, though new HIV diagnoses are declining among this population (CDC, 2018). PLWHA who are over 50 years of age consists of three groups: longtime survivors, those who are newly diagnosed, but not newly infected, and newly infected individuals. While longtime survivors proportionally represent the largest group, the remaining two groups continue to grow. In 2015, individuals aged 50 and over accounted for 6,725 (17 percent) of the 39,513 HIV diagnoses in the U.S. Among those diagnosed after the age of 50, individuals between 50 and 54 years of age accounted for $45 \%$ of HIV diagnoses (CDC, 2015). In 2014, the Center for Disease Control (CDC) reported that 40 percent of individuals diagnosed at age 55 and above already suffered from late stage infection (AIDS) at the time of diagnosis. Such drastic disease progression is due to late diagnosis and can be exasperated by an older individuals' false sense of safety in terms of their HIV risk. Older adults may assume that safe sex practices such as condoms are mainly for contraceptive purposes and, therefore, unwarranted at their age. Additionally, medical 
providers rarely test older adults for HIV, assuming they are not engaging in HIV risk behaviors. Therefore, providers are largely unprepared to address the issue among this population. In 2014, the CDC reported that 39 percent of all HIV-related deaths occurred among persons aged 55 and over. Studies have shown that health care providers are often hesitant to conduct a sexual history with older adults, and older adults are reluctant to acknowledge their own risk behaviors. HIV-related symptoms in older adults could be mistaken for age-related illnesses, since several symptoms of HIV, such as declining immune function and changes in physical and mental condition, can resemble age-related conditions. Older adults, themselves, as well as their health care providers, may associate such symptoms with the aging process and fail to test for an HIV infection (CDC, 2015). Given the lack of understanding that HIV may be the reason for symptoms, older adults may not reveal risk behaviors to their health care providers.

\section{Older African American Adults and HIV}

Even though strides have been made in understanding the virus and in developing effective medications, the rate at which the African American population is impacted by HIV is alarming. In 2018, African Americans, who represented 13 percent of the population accounted for $41 \%$ of all the new human immunodeficiency virus (HIV) infections (CDC, 2018). The CDC reports that since 1981, more than 290,000 African Americans have died from AIDS. Although the death rate has decreased dramatically over the last three decades, more than 7,000 African Americans died of AIDS in 2017 (CDC, 2018). In 2018, African Americans made up 47 percent of new AIDS diagnoses and accounted for 30 percent of new HIV diagnoses among older adults ( $>50$ years of age) (CDC, 2018). The prevalence of older African American adults living with HIV suggests that older African American adults are more prone to HIV/AIDS infection than their racial counterparts (CDC, 2016). 
The literature concerning HIV/AIDS and African Americans has predominantly focused on studies about HIV risk-taking behaviors, especially among adolescent and young adults (Jolly, Mueller, Chen, Alston et al., 2016; MacQueen, Chen, Jolly, Mueller et al., 2015; Martos, Valera, Bockting \& Wilson, 2016; Shilo \& Mohr, 2015; Stock, Gibbons, Peterson \& Gerrard, 2013; Wallace, McLellan-Lemal, Harris \& Townsend, 2011; Whiteley, Brown, Swenson, Romer et al., 2011) and less so about older individuals (Braithwaite \& Arriola, 2003; Harris, Crawford, Kerr, Thomas \& Schmidt, 2020; Krebs \& Simmons, 2002). While research documents the impact HIV/AIDS has caused on African Americans as a population, the complex issues facing older AAPPLWHA are largely undocumented.

\section{Incarceration and HIV/AIDS}

In contrast to the general population, the HIV rates among incarcerated individuals are disproportionally high. The U.S. Bureau of Justice Statistics (BJS) reports that 2.2 million individuals are currently incarcerated (U.S. Bureau of Justice Statistics, 2015). Approximately $1.5 \%$ of all inmates in U.S. state and federal prisons are HIV positive (Fazel \& Baillargeon, 2011). Not only is the HIV rate among incarcerated individuals 5 to 7 times that of the general U.S. population, but the rate of confirmed AIDS cases in incarceration settings is 2.5 times higher (AIDSinfonet, 2014; Iroh, et al., 2015). When referring to U.S. incarceration institutions, a distinction between prisons and jails is necessary. Prisons are long-term facilities, typically run by the state or federal government that hold inmates and felons who have been given at least a one-year sentence. Jails, on the other hand, are with a few exceptions, short-term facilities (typically less than one year) operated by counties, holding incarcerated individuals awaiting their trial and/or sentencing (U.S. Bureau of Justice Statistics, 2015). 
In a systematic review on the HIV care cascade (sequential steps of HIV care from initial diagnosis to the goal of viral suppression) before, during, and after incarceration, Iroh and colleagues (2015) found that the procedures for HIV testing vary greatly. Only about half of U.S. state prison systems have policies that mandate HIV testing for inmates (Maruschak et al., 2009). In U.S. jails, HIV testing is even less common partly due to the quick turnover rate compared to state prisons. Despite the CDC's recommendations to implement at least opt-out testing (meaning individuals explicitly decline HIV testing) nationwide in correctional facilities, only about half of the U.S. state prison systems have implemented policies that mandate HIV testing (Beckwith, Nunn, Baucom, Getachew et al., 2012). As mentioned previously HIV prevalence and incidence is only documented in 19 states (CDC, 2015; Center for HIV Law and Policy, 2017). Besides a basic health screening the state of Kentucky does not routinely test inmates for HIV in any correctional setting including prisons or jails (CDC, 2015; Center for HIV Law and Policy, 2017; Kentucky Department of Corrections, 2017). Paradoxically, jails and prisons create an opportunity for much needed HIV care and health services for those who are medically underserved. Previous studies confirm that incarceration provides opportunities for HIV testing, treatment and linkage to care (Iroh et al., 2015).

Considering that one in seven PLWHA (Persons living with HIV/AIDS) in the U.S. passes through correctional facilities each year, correctional facilities are critical sites for HIV testing and treatment (Rich et al., 2013).

Contrary to the popular belief that incarcerated individuals get infected with HIV in correctional facilities, studies have reported that HIV transmission in correctional facilities is indeed low (Braithwaite \& Arriola, 2003; CDC, 2006; Hammet, 2006; Krebs, 2006; Krebs \& Simmons, 2002; Johnson et al., 2009). Yet, the overall lack of routine testing may greatly underestimate these numbers. Previous studies report that most HIV 
positive individuals contract the disease prior to incarceration (AIDSinfonet, 2014; Beckwith et al., 2010; Juergens et al., 2011; CDC, 2013; Dolan, Wirtz, Moazen, Ndeffo et al., 2016; Fogel, Crandell, Neevel, Parker et al., 2015). Nonetheless, incarcerated individuals are at higher risk of acquiring and/or transmitting the disease to others during incarceration. Existing literature confirms high levels of HIV risk behaviors in incarceration settings that increases the risk of disease transmission (Beckwith et al., 2010; Harawa, Bingham, Butler, Dalton et al., 2009; Maruschak, 2012; McClelland, Teplin, Abram, \& Jacobs, 2002). Risk behaviors include Injection Drug Use (IDU), minority status, a history of mental illness, sexual activity, a history of STDs, men having sex with men (MSM), and Black men having sex with men (BMSM) as positive predictors of HIV infection (Altice, Marinovich, Khoshnood, Blankenship et al., 2005; Rosen, Schoenbach, Wohl, White et al., 2009a; Rosen, Schoenbach, Wohl, White et al., 2009). Zawitz and Parisot (2013) highlight the issue of unreported sexual activity and emphasize the high frequency of sexual relations regardless of sexual conduct policies prohibiting all forms of sexual contact. Sexual relations among incarnated individuals are high-risk in terms of HIV transmission, since most correctional facilities prohibit the distribution of condoms (McCleeland et al., 2009b; Zawitz et al., 2013). The fact that correctional facilities prohibit all sexual contact among individuals does not change the fact that sexual relations among incarcerated persons are a reality (Braithwaite et al., 2002; May \& Williams, 2002). Consequently, HIV in the context of incarceration settings remains a serious health issue that needs to be addressed.

\section{African Americans and Incarceration}

African Americans are vastly overrepresented in any detention setting with 37 percent of incarcerated individuals in local jails (Minton \& Golinelli, 2014) and 36 percent of individuals at the state and federal level representing this population (U.S. 
Census Bureau, 2018, Carsen, 2014). African American men make up 36 percent of the U.S. population of men in jail and 37 percent of the U.S. population of men in prison (Mayer et al., 2002; U.S. Census Bureau, 2018; Carsen, 2014). African Americans do experience significant racial disparities (Lyons, Osunkoya, Anguh, Adefuye et al., 2014; Minton et al., 2014; Maruschak, 2015). Racial disparities are multifaceted, and the high incarceration rate of African Americans cannot solely be explained by one factor (Juergens et al., 2011; Khan et al., 2015). A number of social conditions impact this statistic including limited access to sustainable and living wage, limited economic opportunities, and institutional racism. Residing in communities disproportionally affected by the dealing and use of illicit drugs, increases the incarceration rates of African Americans throughout the U.S. (Juergens et al., 2011; Khan et al., 2015; Weitzer, 2017). Both African American men and women are incarcerated at higher rates than Caucasians in every age group. African American males are about twice as likely as Hispanics and six times more likely than Caucasian males to be incarcerated in their lifetime. Similar findings are observed among African American women. While 3.6\% of African Americans women will enter a correctional facility at least once during their lifetime, statistics show that only $1.5 \%$ of Hispanic and $0.5 \%$ of Caucasians females will be incarcerated (U.S. Department of Justice, 2011). Although HIV and incarceration affect all ethnicities, the HIV rates are highest among incarcerated African Americans (CDC Surveillance Report, 2010; Brinkley-Rubinstein, \& Turner 2013). Brinkley-Rubenstein \& Turner (2013) used ethnography to explore the impact of incarceration on health in twelve HIV positive African American males. The authors suggest that HIV and incarceration stigma, substandard medical care, and delayed access to care (e.g., HIV medication) affect the health of African Americans greater than their racial counterparts. Even post-release, the African American participants described a worsening of their 
health due to HIV and incarceration stigma, loss of social support, and delay or inability to access HIV-related services. These findings have been supported by James-Borga (2013) and point to the syndemic connection of incarceration, HIV and African Americans as described by Brinkley-Rubenstein \& Turner (2013).

\section{Incarceration and Older Adults}

Incarcerated individuals aged 50 and older constitute the fastest growing age group of prisoners in the United States, not necessarily because they are entering correctional settings at higher rates than younger individuals, but because they are aging in place while incarcerated. Estimates suggest that by the year 2030, about one-third of the incarcerated population in the U.S. will be 55 years of age or older (Rich et al., 2013). Chettiar, Bunting and Schotter (2012) reported that the aging prison population grew on average by 145 percent across sixteen southern states (including Kentucky) between 1997 and 2007. This is a more rapid increase than the total prison population in those states. In Kentucky, the number of older incarcerated individuals (50 years and over) increased 136.8 percent compared to a 54.34 percent increase in the total prison population (Kentucky Department of Corrections, 2017).

Older incarcerated individuals living with HIV face unique challenges. Older incarcerated individuals' biological aging is often accelerated, as documented in the literature. Further, being older increases the susceptibility of experiencing injuries during falls, enhanced sensitivity to heat and cold, vision and hearing changes, and a greater potential of contracting other contagious diseases such as tuberculosis (Beckwith et al., 2010; Department of Corrections, 2018). If living with HIV creates unique challenges for older adults, adding the institutional environment of incarceration can further advance the aging process. Studies have shown that persons living with HIV/AIDS experience an “accelerated" or "accentuated" aging process (Martin et al., 2010; Pathai et al., 2013) 
therefore, incarcerated PLWHA who are 50 years and older may experience accelerated biological aging compared to their peers without incarceration histories. As such, older incarcerated PLWHA are at increased risk of experiencing age-related multiple illnesses (e.g. dementia) as well as HIV-associated conditions such a hepatitis B, hepatitis C and liver disease all of which require treatment and can complicate HIV treatment (Rich et al., 2013; Brothers et al., 2014;). Not surprisingly, studies have shown that HIV/AIDSrelated deaths among incarcerated individuals in a state prison are the highest among African Americans and individuals' aged 50 and above (U. S. Department of Justice, 2015). The prevalence of older AAPLWHA with incarceration histories warrants the need to explore their incarceration experiences and the implications for HIV testing, disclosure experiences and engagement in care in older African American adults with HIV. Additionally, there is a paucity of research that addresses the lived experiences of older persons living with HIV who have incarceration experiences (Tietz, 2013). Aging trends suggest that research about this population will have far reaching consequences for the coming decades.

\section{Intersection of Incarceration, Health Care and HIV}

Paradoxically, incarceration settings do create an opportunity for much needed HIV care and health services to those who are medically underserved. Whether the opportunity actually provides treatment is debatable. The Eighth Amendment's prohibition of "cruel and unusual punishment" guarantees every incarcerated individual in the U.S. a right to health care. Yet several studies (Clemmit, 2007; Wilper, Woolhandler, Boyd, Lasser et al., 2009) report that it is not uncommon for incarcerated individuals suffering from chronic physical illnesses to be denied appropriate health care. One of the many factors contributing to the lack of adequate medical care is the lack of standards for the delivery of health care across the criminal justice system. Depending on the 
incarceration facility and the type of incarceration setting, the availability of health care varies. Previous studies confirm that incarceration provides opportunities for HIV testing, treatment and linkage to care (Iroh et al., 2015). The remaining question is how services are delivered, for how long and to what benefit of the incarcerated PLWHA.

\section{HIV and Incarceration Stigma}

Despite major advances in HIV research, available effective treatments and nationwide public health education campaigns, HIV still provokes stigma (Mahajan, Sayles, Patel, Remien et al., 2008; Zanoni \& Mayer, 2014). According to UNAIDS, HIV/AIDS-related stigma encompasses a "process of devaluation" of people either living with or associated with HIV/AIDS. Discrimination oftentimes coincides with stigma and is characterized as the unjust and/or unfair treatment of individuals based on their actual or perceived HIV status (UNAIDS, 2013). Zawitz and colleagues (2014) noted that incarcerated PLWHA fear HIV stigma more than disease progression or even death. HIV stigma in correctional facilities is exasperated by the closed-in environment and the lack of privacy, which can lead to isolation, discrimination and an increased vulnerability to physical attacks (Andrinopoulos et al., 2010; Juergens et al., 2011).

HIV stigma affects incarcerated PLWHA throughout the incarceration experience. Starting at intake, PLWHA must disclose their HIV status to a healthcare provider in order to receive HIV care and access to medication. PLWHA detained in jails or other forms of short-term detention encounter a different set of challenges than those in state and federal prisons. Individuals detained for short periods of time often choose not to disclose their status and take the risk of becoming ill rather than exposing themselves to HIV stigma (Juergens et al., 2011; Brinkley-Rubinstein et al., 2013). Short term detention often renders individuals unable to access their HIV medication, which can dramatically impact their medication adherence, viral load, and the ability to take their 
previous combination of ART once released (Althoff, Zelenev, Meyer, \& Fu, et al., 2013; Meyer, Cepeda, Wu, Springer et al., 2014; Small et al., 2009). Outside of the correctional setting, PLWHA can engage in medical care without others' knowing their HIV status. The lack of privacy in the incarceration environment poses a significant disclosure risk. Misinformation regarding the modes of transmission, general HIV knowledge, and stereotypes of who is at risk contribute to stigma and irrational fears (Brinkley-Rubinstein et al., 2013; Khan et al., 2015). HIV infection remains highly stigmatized in society and remains associated with behaviors such as commercial sex work, IDU, MSM and BMSM. In correctional settings, this stigma places PLWHA at an elevated risk for discrimination, assault and even murder (Sprague et al., 2016).

During the early years of the HIV epidemic in the U.S., incarceration facilities had mandatory HIV testing and housing segregation policies in place. Those policies were intended to reduce stigma and prevent transmission, but often resulted in discrimination, increased stigma, and mistreatment of incarcerated PLWHA (Hammet, 2006; ACLUNational Prison Project and Human Rights Watch, 2010). Starting in 1996 with the availability of effective antiretroviral therapy (ART), the number of segregated housing policies for incarcerated PLWHA declined rapidly (Polonsky, Kerr, Harris, Gaiter et al., 1994; Inciardi, 1996; Levy, Wilton, Phillips, Glick et al., 2014; McCarthy et al., 2016; Wright \& Carnes, 2016). By 2010, only Mississippi, South Carolina and Alabama maintained segregation. Mississippi reversed the policy the same year and, in 2012, a federal court ruled that Alabama's segregation policy was violating the Americans with Disabilities Act. South Carolina was the last state to end the policy in 2013. Currently, HIV housing segregation policies have been reversed in state prisons across the U.S. (ACLU-National Prison Project and Human Rights Watch, 2010; Center for HIV Law and Policy, 2017). 
Diagnostic programs and routine HIV testing in incarceration settings have been identified as critical links to care, such as screening for undiagnosed HIV infections, maintaining the health of incarcerated PLWHA, and preventing future disease transmission (Levy et al., 2014; McCarthy et al., 2016). These procedures contribute to the continuity of care of incarcerated PLWHA. Being tested for HIV at a correctional facility may be the first time a person is confronted with their illness and able to engage in care (Rich et al., 2013). Others who suspect they are HIV positive can receive testing and HIV-care for the first time while incarcerated. Regardless of a person's knowledge of their HIV status prior to incarceration, this does not change the important role correctional sites play in HIV testing and treatment (Rich et al., 2013; McCarthy et al., 2016). 


\section{CHAPTER THREE: THEORETICAL REVIEW}

This chapter explains the theoretical context of this study and elaborates on theories related to my research questions as an extension of the literature review. In order to provide a clearer theoretical context for my study, I synthesized the Comprehensive Health Seeking and Coping Paradigm (Nyamati, 1989) framework as a theoretical sensitizing concept to lay a foundation of how the incarceration experiences of older AAPLWHA are contextualized within it. Since the population under study was older AA adults living with HIV, and the purpose was to gain a deeper understanding of their incarceration experiences, the CHSCP assisted as a starting place in which to conceptualize the study. I present an emerging conceptual framework (Figure 1) before concluding with the principles of Symbolic Interactionism (SI) to explain how this methodological perspective relates to the proposed study.

A well-defined theoretical perspective is crucial when engaging in qualitative research. Theory driven thinking and acting is woven throughout the research process from the selection of a potential topic, the development of research questions, conducting the literature review, analyzing data, and drawing implications. Kerlinger (1986) defined theory as "set of interrelated constructs definitions, and propositions that presents a systematic view of phenomena by specifying relations among variables, with the purpose of explaining and predicting phenomenon (p. 9)." Theory is defined differently by academic scholars and scientific philosophers and based on the nature of the study theoretical assumptions vary. For example, in a grounded theory study, theory emerges from the data analysis process; however, the use of a theoretical framework as a 
sensitizing concept can guide the analysis (Glaser, 1978; Padgett, 2004). Blumer (1954) first introduced sensitizing concepts by contrasting them with definite concepts. Blumer (1954) explained, "Whereas definite concepts provide prescriptions of what to see, sensitizing concepts merely suggest directions along which to look" (p. 7). According to Mertens (1998), the theoretical framework of a study "has implications for every decision made in the research process" (p. 3). Often, qualitative researchers utilize sensitizing concepts as "points of departure" (Charmaz et al., 2003 p. 259) and as a lens through which to view the research problem (Glaser, 1978; Padget, 2004; Patton, 2002). Charmaz (2003) emphasized that sensitizing concepts are merely starting points and by no means a tool to bypass or direct the analysis that emerges from the data.

In this study, I considered the CHSCP (Nyamathi, 1989) as a sensitizing concept to inform this research. Variables such as socio-demographic factors, situational factors, or personal resources situate and describe an individual's environment and affect health seeking and coping responses. I identified variables that related to and were relevant to older African American adults living with HIV and their incarceration histories. Identifying possible domains and categories of the CHSCP served as a starting point from which the data was analyzed. As consistent with constructivist grounded theory, the purpose of this study was not to test, refine or improve the CHSCP framework. During the data analysis as concepts emerged the sensitizing concept of the CHSCP might need to be supplemented or even displaced (Padgett, 2004). The main purpose of the CHSCP along with the literature on the African American experience, aging, HIV, and incarceration was to inform this research.

\section{The Comprehensive Health Seeking and Coping Paradigm}

Nyamathi's (1989) CHSCP provides a framework acknowledging that incarceration experiences of older AAPLWHA are influenced by several individual and 
environmental factors. Within the CHSCP, those can be classified as socio-demographic factors (e.g. age, education, marital status and socioeconomic resources) personal factors (e.g. perceived stigma, disclosure experiences, incarceration setting and duration) and social factors (social support, engagement in care). Nyamathi's CHSCP combines Lazarus \& Folkman's (1984) conceptualization of stress, appraisal and coping, along with Schlotfeldt's (1981) conceptualization of health seeking behaviors to provide structure through which the relationship of coping and health status can be understood.

\section{Older AAPLWHA with Incarceration Experiences and the CHSCP}

The CHSCP posits that personal and environmental factors, combined with one's cognitive appraisal of their situation, influences health-seeking behavior and engagement in care. Personal and environmental factors for older AAPLWHA include conditions such as education, employment, incarceration, income, job security, health services, access to services, as well as housing, social exclusion, and stigma (Braveman \& Gruskin., 2003; Raphael, 2009, Sheilham, 2009). Considering the incarceration experiences of older AAPLWHA, this indicates that imprisonment influences both health seeking and engagement in care for older AAPLWHA which can create, change and/or and maintain health disparities. In the context of HIV infection and health disparities among older AAPLWHA, socio-demographic and situational factors are involved in creating vulnerability that can contribute to increased risk of HIV transmission and/or compromise the ability to engage in HIV care. Regardless of whether the person was infected prior to or during the incarceration, the physical environment of incarceration can exacerbate health disparities greatly. 


\section{Incarceration Experiences of Older AAPLWHA: An Emerging Conceptual Framework}

Utilizing the tenants of the CHSCP, Figure 3.1 displays an initial conceptual framework for the proposed study and outlines the HIV care continuum and the major concepts as they relate to older AAPLWHA and their incarceration experiences. The CHSCP falls into three overlapping major domains: The environment (e.g. contextual environment including incarceration context, the health care environment including HIV clinic factors, system factors and provider factors), patient characteristics (predisposing factors, enabling factors, perceived need), and behavioral and psychological processes. The environment includes immediate and visible circumstances in which older AAPLWHA live such as incarceration, poverty, unemployment or lack of insurance. These factors can significantly impact an individual's circumstances and health but fall outside of a person's control. 


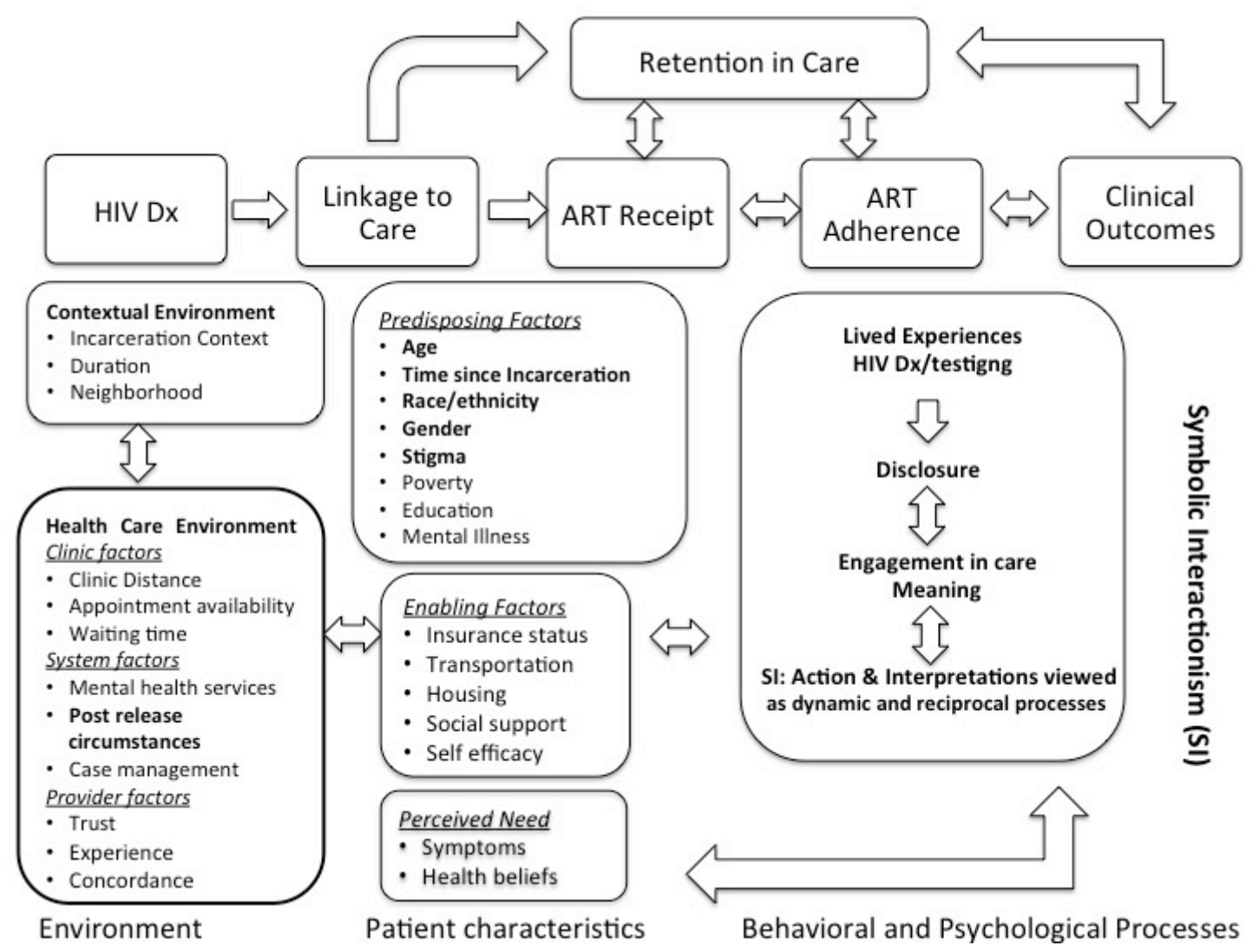

Figure 3.1 Incarceration Experiences of older AAPLWHA an Emerging Conceptual Framework

The potential negative effects of the environment on older AAPLWHA can accumulate over their lifetime and become more apparent as individuals age. The interplay of incarceration experiences with the environment and patient characteristics may affect the behavioral and psychological processes and be experienced differently among older AAPLWHA compared to their younger counterparts with incarceration histories, as well as their similar aged community dwelling peers without incarceration histories. The environment and individual patient characteristics can be examined through qualitative data (i.e. identifying the behavioral processes of older AAPLWHA) and quantitative data, collected during the original study. The original study obtained personal, socio-demographic and clinical characteristics including, date of birth, HIV diagnosis date, race/ethnicity, marital status, education, gender, sexual orientation, 
residence, employment status, income, insurance status, AIDS diagnoses, history of antiretroviral therapy use and adherence, substance abuse history, alcohol use history, mental health diagnoses, CD4 cell counts, and viral loads. Additionally the original study utilized three measures: 1) the Berger HIV Stigma Scale (Berger, 2001), 2) the Perceived Stress Scale (PSS) (Cohen, Kamarck \& Mermelstein, 1994) and 3) the Engagement with Healthcare Providers (HCP) Scale (Bakken, Holzemer, Brown, Powell-Cope, Turner et al., 2000).

\section{Symbolic Interactionism}

CGT is underpinned by the principles of Symbolic Interactionism (SI) and highlights the significance of understanding a situation from an individual's point of view (Charmaz, 2014; Strauss \& Corbin 1998; Glaser \& Strauss, 1967). Symbolic Interactionism is a perspective that allows for new views of meanings related to actions and events and is not an explanatory theory determining variables or certain outcomes. SI was initially introduced by Horton Cooley and George Herbert Mead in the early $20^{\text {th }}$ century. Herbert Blumer (1969), a student of Mead's, expanded SI as a sociological perspective and theoretical approach examining the interaction between small groups, individuals, or individuals and objects. This interaction process is a meaning making process whereby shared symbols are used to communicate meaning. SI is a useful lens to consider the data of this study since it allows the researcher to view action and interpretations as reciprocal and dynamic processes between the older AAPLWHA and their environment (see Figure 3.1). Further, SI acknowledges that individuals, such as older AAPLWHA, act in response to how they view situations such as incarceration experiences. SI views older AAPLWHA not only as active participants that engage in activities in their world (e.g. incarceration experiences), but also as a group/individuals with subjectivity and unique viewpoints. The incarceration experiences of older 
AAPLHWA viewed through a SI lens, acknowledges that both HIV and incarceration stigma are present on an individual, societal and collective level (Charmaz, 2014).

Connecting the theory to the method, the use of CGT is especially pertinent given that the questions posed sought to understand the lived incarceration experiences of older AAPLWHA and explored the phenomenon of incarceration experiences in older AAPLWHA in terms of their meaning and context. Explanations derived from the qualitative data provided an in-depth understanding and descriptions of how participants' lived incarceration experiences construct their realities (Charmaz, 2014). In addition to embracing the tenants of CGT, this study utilized situational analysis, a recent supplement to grounded theory (Clarke, 2003, 2005, 2009; Clarke, Friese \& Washburn, 2015). Clarke proposed that social "situations" should form the unit of analysis and introduced three sociological modes (situational, social world/arenas, and positional maps) to analyze data (Creswell \& Poth, 2017).

This theoretical review has identified that there is currently no theory that explains the meaning making process for formerly incarcerated older AAPLWHA. It is notable that HIV stigma has not been addressed within Symbolic Interactionism framework (Link, Wells, Phealm \& Yang, 2015; Åsbring, \& Närvänen, 2002; Roe, Joseph \& Middleton, 2010). Current theory, such as the Symbolic Interaction Stigma framework (Link et al., 2015) describes how individuals predict what others might think of them due to a potential stigmatizing status. This 'symbolic interaction' (Link, 2015) takes place before an interaction occurs and guides future behavior. For older AAPLWHA their potential stigmatizing status, such as being HIV positive or having an incarceration history may guide future health seeking behavior (i.e. disclosing HIV status, engagement in care) and points to the potential importance of considering symbolic interactions (which can take place before an actual interaction occurs) in the meaning making 
processes for older AAPLWHA. In conclusion, qualitative inquiry was a fitting approach to conduct this study and the CHSCP was introduced as sensitizing concept. Together, with SI the CHSCP informed the development of an emerging conceptual framework that was used as a point of departure for this study. 


\section{CHAPTER FOUR: METHODOLGY}

This chapter describes the study's research methodology and includes discussions of the following areas: (a) rationale for qualitative research design, (b) social constructivism and rationale for constructivist grounded theory methodology, (c) description of the original study, (d) description of the research sample, (e) overview of research design, (f) methods of data collection, (g) analysis and synthesis of the data, (h) ethical considerations, and (i) rigor, trustworthiness and positionality and reflexivity statement.

\section{Rationale for a Qualitative Research Design}

Qualitative inquiry is exploratory in nature and questions the existence of an objective reality (Hesse-Bieber, 2017; Mason, 2002). It is rooted in a constructivist philosophical position, meaning it is concerned with how the complexities of the social world are experienced, interpreted and understood in a certain context and at a particular point in time. Qualitative inquiry fosters a holistic rather than reductionist understanding when entering the world of others (Corbin \& Strauss, 2015; Hesse-Bieber, 2017; Mason, 2002). Denzin and Lincoln (2011) define qualitative research as a "set of interpretative, material practices" (Denzin \& Lincoln, 2011, p. 3) through the exploration of phenomena and the meanings people ascribe to them.

Creswell (2016) adopts significant elements of Denzin \& Lincoln's definition and points toward the importance of the research design, specifically the use of the five approaches to inquiry by emphasizing the emergent nature of qualitative approaches and centrality of the voices of participants as well as the reflexivity of the researcher (i.e. 
grounded theory, phenomenology, narrative research, ethnography and case study) (Creswell, 2016).

Qualitative research allows the researcher to enter the world of participants and emphasizes discovery and description. The objectives of qualitative inquiry are generally focused on extracting and interpreting the meaning of experiences (Denzin \& Lincoln, 2011; Merriam, 1998). For the purpose of this study, a solely quantitative methodology was not well suited to address the research questions and purpose of the study. Solely designating and distinguishing the relationship between variables, as it is the aim of quantitative methods, would not produce the rich data necessary to understand incarceration experiences for older AAPLWHA, and how the interpretation of these experiences create meaning related to stigma, disclosure, and engagement in care.

The key features and assumptions representing a qualitative stance align well with the purpose of this study. These key features include (a) understanding of the processes by which events and actions take place, (b) developing contextual understanding, (c) facilitating interactivity between researchers and participants, (d) adopting an interpretive stance, and (e) maintaining design flexibility (Mason, 2002; Miles, Huberman \& Saldana, 2013; Ormston, Spencer, Barnard \& Snape, 2014).

\section{Social Constructivism and Rationale for Constructivist Grounded Theory}

\section{Methodology}

The philosophical assumptions that underlie all qualitative research are embedded within interpretative frameworks (Denzin \& Lincoln, 2011). By utilizing Charmaz's (2003) version of grounded theory, I assumed the "relativism of multiple social realties" (p. 215) and emphasized the co-construction of knowledge through interpretative understandings of meaning. Social constructivism, as described by Charmaz (2014) views knowledge as constructed as opposed to created. I have aligned myself with a 
social constructivist framework and acknowledged that this study is based on the belief that multiple realities are constructed through lived experiences as well as through interactions with others (Charmaz, 2014). This was be accomplished by staying grounded in the data and by relying strongly on participants' viewpoints and experiences; the belief that reality is co-constructed between the research participants (older AAPLWHA) and myself as the researcher was assured. I admitted to the value laden nature of this study and "positioned myself" in it through actively reporting my own biases and values throughout the research process, thereby increasing the rigor of trustworthiness of this study (Creswell, 2016).

Within the framework of a qualitative approach, the proposed study is most suited for a constructivist grounded theory design to gather, analyze and synthesize data. In contrast to classic grounded theory, CGT allows a focus on the psychological and behavioral processes of older AAPLWHA and their incarceration experiences. Charmaz (2003, 2014) developed CGT as an alternative to classic (or Glasserian) grounded theory (Glaser, 1978, 1998, 2001, 2003, 2005, 2011) and Straussian grounded theory (Strauss \& Corbin 1990, 1998). Grounded theory methodology was first introduced by Glaser and Strauss (1967) and developed further by Glaser, Strauss, Strauss and Corbin, Charmaz, Clarke and several others. The nature of grounded theory is to develop theory from data, which is systematically gathered and analyzed during the research process (Strauss \& Corbin, 1998). Grounded theory methods consist of a systematic approach to inquiry with several key strategies (i.e. inductive, comparative, iterative, interactive) for conducting inquiry (Charmaz, 2014). Data collection, analysis and the subsequent development of theory are closely related. Since its development grounded theory as diverged in several directions. While Strauss and Corbin allow the formulation of the research problem in the beginning of the research process, Glaser's training in survey 
research gave the method its systematic approach, positivist proclivities, and procedural language. Original grounded theory was rooted in positivism and did not fully account for the reflectivity of the researcher in the study process (Charmaz, 2014). Constructivist grounded theory builds on Glaser and Strauss's (1967) original inductive, comparative, emergent and open-ended approach but integrates developments in qualitative inquiry, addresses criticism raised in grounded theory methodologies and moves grounded theory into critical and interpretive inquiry. Charmaz (2003) proposed a version of grounded theory that "assumes the relativism of multiple social realities, recognizes the mutual creation of knowledge by the viewer and viewed, and aims toward an interpretative understanding of subjects' meanings" (p. 250). "Relativism of multiple social realities" allows for capturing multiple truths represented by multiple older AAPLWHA experiences (Martin, 2006, p. 206). The co-construction of data between the researcher and the participants is another key tenant of CGT (Charmaz, 2003, 2014) utilized in this study. As consistent with CGT, this study focuses on "interpretative understanding of subjects' meanings" (Charmaz, 2003, p. 250) and aims to understand older AAPLWHA incarceration experiences from an interpretative and descriptive approach.

The use of CGT was especially pertinent given that the questions posed sought to understand the lived incarceration experiences of older AAPLWHA and explored the phenomenon of incarceration experiences in older AAPLWHA in terms of their meaning and context. Explanations derived from the qualitative data provided an in-depth understanding and descriptions of how participants' lived incarceration experiences constructed their realities (Charmaz, 2014). In addition to embracing the tenants of CGT this study utilized situational analysis, a recent supplement to grounded theory (Clarke, 2003, 2005, 2009; Clarke, Friese \& Washburn, 2015). Clarke proposes that social "situations" should form the unit of analysis and introduces three sociological modes 
(situational, social world/arenas, and positional maps) to analyze data (Creswell \& Poth, 2016).

\section{Description of the Original Study}

The original mixed methods pilot study, titled "African American Older Adults Living with HIV: Exploring Stress, Stigma and Engagement in Care" was conducted in 2016. The purpose of this study was to explore the relationships among stress, stigma, and engagement in care among older African Americans living with HIV. The study consisted of three phases of data collection: (1) a cross-sectional survey; (2) qualitative in-depth interviews; and (3) one final member checking focus group.

Survey Measures: Demographic and clinical characteristics were obtained via self-report. Demographic data included age, gender, education, sexual orientation, relationship status, employment status, history of incarceration, history of homelessness, and history of substance use. Clinical data included, AIDS diagnosis, duration of HIV, mental health conditions, history of comorbid conditions, insurance status and history of missed clinic visits.

Berger HIV Stigma Scale: The Berger HIV Stigma Scale (2001) was used to measure stigma. The Berger's stigma scale has 40 items and respondents rate on a 5point likert scale from "Strongly Disagree" to "Strongly Agree." The items were summed to create an overall stigma score and ranges from 0 to 160 . In addition, subscales included personalized stigma (related to the consequences of other people knowing their HIV status), disclosure concerns, negative self-image related to HIV status, and public attitudes regarding HIV. Coefficient alphas of .96 for the 40- item instrument provided evidence of internal consistency reliability.

Perceived Stress Scale (PSS): Stress was measured using the Perceived Stress Scale (PSS) (Cohen et al., 1994). The PSS is a 30-item scale that includes a number of 
direct queries about current levels of experienced stress, and respondents are asked to determine how often they have experienced stressful life events. The scale provides a total score for perceived stress and ranges from 0 to 40 . Coefficient alpha reliability ranged from .84 to .86 (Cohen et al., 1994).

Engagement with Health Care Providers (HCP) Scale: Engagement with health care providers was measured using the Engagement with Health Care Providers (HCP) Scale. The HCP is a 13-item scale, where respondents indicate the nature of their interactions with their health care provider on a 4-point scale $(1=$ always true and $4=$ never true). The items were summed (range $=13$ to 52 ), with lower scores indicating more engagement. Cronbach's alpha reliability estimates ranged between 0.90 and 0.96 (Bakken et al., 2000).

Composite of Engagement in HIV Care: The Composite of Engagement in HIV Care was used to measure engagement in HIV care and to supplement the HCP Scale. This 7-item scale assesses engagement in care through self-reports of attending appointments, medical knowledge, and antiretroviral (ARV) adherence (Saberi \& Johnson, 2015). The 7 items are summed and scores range from 0 to 7 ; scores were categorized in low engagement $(0-4)$, moderate engagement $(5-6)$, and high engagement (7).

Qualitative In-Depth Interviews: In-depth interviews were conducted with each participant with the purpose of qualitatively exploring barriers and facilitators to engagement in care (complete interview guide listed in appendix C). Consent was obtained prior to the beginning of the interview. Each interview lasted between 50 and 90 minutes and was audio recorded. A follow-up interview was conducted if additional information was needed. 
Focus Group: In order to increase the trustworthiness and credibility of the combined analysis from the first two phases of data collection, a member checking focus group (Creswell \& Miller, 2000; Merriam, 1998) was conducted in the final phase of the study. The maximum variation approach to purposive sampling was used to select six AAPLWHA in terms of level of stigma, stress and engagement in care (based on quantitative and qualitative results). The focus group was used as an opportunity to present the findings of the study to the participants, to gain valuable feedback, and ask further questions in areas where the emerging context specific framework/findings were limited.

Study Sample: Participants were eligible for the study if they identified as Black or African American and were 50 years of age and older. Individuals were included in the study if they had confirmed diagnosis of HIV, were able to speak and understand English, and were able to provide consent to the study. A total of 35 older AAPLWHA participated in the original study.

Recruitment: African American adults, 50 years and over, living in the Louisville area were recruited using purposive sampling strategies. Purposeful sampling techniques set the tone for in-depth qualitative analysis by systematically representing a variety of perspectives on the topic of the study, i.e. stress, stigma and engagement in HIV care. Flyers concerning the study were placed at HIV clinics, AIDS Service Organizations (ASOs), and notices were published in HIV consumer newsletters. In addition, the PIs held information sessions with the staff of the Kentucky HIV/AIDS Care Coordinator Program (KHCCP) and House of Ruth (a local ASO) in an effort to enhance clinic participation in the study. An initial phone screening ensured that all participants met the inclusion criteria. Following the screening, eligible participants were invited to complete a survey. Surveys were administered by trained research assistants and investigators. 
After completion of the survey, an in-depth qualitative interview was conducted.

Interviews lasting between 50 and 90 minutes, utilized an in-depth qualitative interview guide. Following the interview, the digital audio file was professionally transcribed. Participants received a $\$ 35$ gift card for their participation.

I was a research assistant throughout the development of the study, data collection and analysis. The high prevalence of incarceration experiences among participants was not anticipated. As a result, the need for the proposed dissertation research became apparent and focuses solely on the incarceration experiences of older AAPLWHA.

\section{Description of the Research Sample}

The sample for this study was selected from the original study ("African American older Adults Living with HIV: Exploring Stress, Stigma, and Engagement in HIV Care"). The first wave of data was drawn from twenty-two out of the 35 participants from the original study. Those twenty-two participants had incarceration histories and discussed those during the initial qualitative in-depth interviews which explored stress, stigma and engagement in care. For the second wave of data, I employed theoretical sampling and identified nine participants for additional in-depth qualitative interviews for this dissertation research. Seven of those were successfully recruited. All materials for the original, as well as this study were reviewed by the Institutional Review Board at the University of Louisville prior to recruitment (Appendix D).

For the purpose of this study incarcerated individuals were persons who were confined in a prison or jail. This also included halfway houses, weekend programs, and other facilities in which persons were incarcerated overnight. To be eligible for this study, participants needed to identify as Black or African American, be 50 years of age or older, have a confirmed diagnosis of HIV, have an incarceration history, were currently seeking HIV care, were able to speak and understand English, and were able to provide 
consent to the interview. Defining an older adult as being age 50 and older is appropriate based on the precedent set by other studies that emphasize "accelerated" or "accentuated" biological aging process (Pathai, et al., 2013) among adults living with HIV (Babiker et al., 2001; Egger, May, Chene, Phillips et al., 2002; Nogueras, Navarro, Anton, Sala et al., 2006), as well as among older incarcerated individuals (Aday, 2003; Beckwith et al., 2010; Falter ,2006; Hayes, Burns, Turnbull, \& Shaw 2012; Rich et al., 2013; Spaulding et al., 2011).

\section{Sampling Strategy}

\section{Sampling Strategy}

\section{Tier 1: Original Study $(\mathrm{N}=35)$}

- 3 Phases of data collection

- (1) Cross sectional survey

- (2) Qualitative in-depth interviews

- (3) Member checking focus group

\section{Tier 2: Dissertation Research $(\mathrm{N}=22)$}

- $1^{\text {st }}$ Round secondary qualitative data analysis

- $2^{\text {nd }}$ Round of Interviews ( $\mathrm{N}=7$ )

- Information rich cases actively recruited for additional interviews

Figure 4.1 Sampling Strategy

A purposive sampling strategy was employed for this study. This study was a deeper investigation of the 22 participants ( 35 total participants in the original study) who had incarceration histories and discussed those during the initial qualitative in-depth interviews. From those original 22 participants seven information-rich cases were purposefully selected and additional in-depth qualitative interviews conducted. The aim 
was to gain a deeper understanding of the incarceration experiences of older AAPLWHA. The information obtained through the seven in-depth qualitative interviews subsequently forms the basis for the findings of this study as well as the 22 interviews of the original study where participants discussed their incarceration experiences. Three out of those seven participants additionally participated in a member checking-focus group. The intent of this study was to understand the in-depth incarceration experiences of older AAPLWHA throughout Louisville, Kentucky. I considered this number of participants sufficient since this CGT study aimed to gain a deeper understanding of the incarceration experiences and participants' understandings of their meanings. Theoretical saturation was reached at the end of the second wave of interviews, since no new information was discovered as I was moving back and forth between data collection and analysis.

\section{Overview of Research Design}

Summary of the steps that were needed to carry out this research:

1. The data collection for this study occurred simultaneously during the original study between May and December of 2016. During this time, a deeper investigation utilizing semi-structured, in-depth interviews were conducted with a total of 22 participants that had incarceration histories. Seven participants out of those 22 aforementioned were selected for additional indepth qualitative interviews exploring the incarceration experiences.

2. I developed the interview guide, and simultaneously engaged in a process of interviewing, memo writing and analyzing the data.

3. Following the defense of the initial proposal I began the data analysis. Interview transcripts and memos were analyzed using constructivist grounded theory techniques as well as situational analysis. 


\section{Methods of Data Collection}

Interested participants contacted the research team via contact information on fliers or received the information through word of mouth. During an initial phone screening the initial study was explained and interested participants were screened to ensure that they met the inclusion/exclusion criteria. Following the screening, eligible participants were invited to participate. Participants were asked to provide written informed consent, with the written understanding that multiple interviews might be requested. All interviews were conducted in private offices at the $550 \mathrm{Clinic}$, the KCCP, or in a private location of the participants' choice including their own home. All interviews were tape recorded and transcribed verbatim. Each interview was between 60 and 90 minutes in length. Participants received an additional \$20 gift card upon completion of the additional interview(s).

\section{In-Depth Interviews}

In-depth interviews are a crucial tool in qualitative research. Charmaz (2014) views interviews as "emergent interactions" that allow participants to describe and reflect on their own lived experiences in a unique way. SI emphasizes that meanings become apparent out of actions and in succession influence actions (Charmaz, 2014). Individuals such as older AAPLWHA construct their selves, society and reality through action and interaction and create and mediate meanings related to their incarceration experiences through engaging in social processes. In-depth interviews are a tool to gather rich and thick descriptions of older AAPLWHAs' incarceration experiences while allowing the participant to share his or her own unique experiences (Denzin \& Lincoln, 2011).

\section{Analysis and Synthesis of the Data}

The audio files were saved onto a password-protected site accessible only to research team members. All identifiers (e.g., names) were electronically removed and the 
de-identified audio files were saved. An inductive approach was utilized to analyze and code qualitative data, however the CHSCP framework was employed as a theoretical lens through which to consider the interview data, and as a sensitizing concept that guided, but not defined the analysis (Blumer, 1954; Charmaz, 2014). The qualitative data analysis was conducted using constructivist grounded theory techniques using Dedoose software as an organizational tool for data analysis (Charmaz, 2014; Dedoose version D 4.5, 2013). Using peer debriefing and consensus building around themes, I built a codebook consisting of code families with definitions (Erlandson, 1993). After the codebook was established, two researchers separately coded the original 22 and seven additional interviews and conferred after all transcripts had been coded. In addition, a Cohen's Kappa test was conducted via Dedoose in order to establish an inter-coder agreement score that is above .90 (Cohen, 1960; Huberman \& Miles, 1994). The Kappa test, along with this iterative process helped to identify segments in which codes did not agree, so that the coders could address and edit the code segments and continue with more accurately coded material (Guest \& McQueen, 2007). The analysis used a continuously emergent process of data collection, data reduction, data display, and data interpretation (Huberman \& Miles, 1994). In summary, data reduction through coding began initially on a line-by-line basis, using phrases as the analytic unit. This detailed analysis generated an in-depth and descriptive depiction of older AAPLWHAs incarceration experiences. The analysis was supported and supplemented by situational analysis (Clarke, 2003, $2005,2009)$ through the creation of three kinds of analytic maps (situational maps, social worlds arena maps, and positional maps) and memos that centered on explaining discourses, key elements, structures and conditions that characterize the incarceration experiences of older AAPLWHA (Clarke, Friese \& Washburn, 2015). 


\section{Ethical Considerations}

All participants of this study were older AAPLWHA volunteering to participate. All 22 older AAPLWHA were interviewed to gain a deeper understanding of their incarceration experiences. There was no harm associated with this study other than that participants may feel emotionally uncomfortable as they recalled their incarceration experiences. Each participant who met the inclusion criteria and agreed to participate completed a consent form. To ensure confidentiality participants could choose a pseudonym or codename with which they were referred to throughout this study. Additionally, all potentially identifying information such as names, age and locations were changed on all the transcripts. The audiotapes, files and interview transcripts were stored in my locked home office on an encrypted file. All participants were informed that they were free to withdraw from the study participation at any time without consequences. Before signing the consent form all participants were given a chance to analyze and discuss the consent form to ensure that they were making an informed decision.

\section{Rigor, Trustworthiness, Positionality \& Reflexivity Statement}

For this study Lincoln and Guba's (1985) five criteria for trustworthiness of a qualitative study were utilized: Credibility, transferability, dependability, and confirmability. Credibility refers to the confidence in the accuracy of the research findings (Lincoln and Guba, 1985). Transferability addresses the ability to apply the research to similar contexts, population, and issues (Padgett, 2004). Dependability is achieved by an ability to repeat the study's methods and a consistency in the interpretation of the findings (Lincoln \& Guba, 1985). Confirmability refers the notion that the findings are based on the participants' narratives and were not shaped by 
researchers biases (Lincoln \& Guba, 1985). This study draws on each of these criteria as described below.

\section{Credibility}

Credibility was achieved through 1) triangulation, 2) member checking, 3) prolonged engagement and 4) peer de-briefing. Triangulation of multiple data sources (interview data, observational data, focus group, existing literature) was utilized to corroborate the evidence (Lincoln \& Guba, 1985; Merriam \& Tisdell, 2015). Member checking occurred for the seven additonal interviews to confirm emerging theoretical categories, and for the focus group that was conducted during the original study. Prolonged engagement in the field allowed for participants to become comfortable with relating to the research team. Many participants felt comfortable with the research team and chose to conduct the interviews in their homes. Phone correspondance throughout recruitment and inclusion of the member checking focus group ensured sufficient time spent with participants in the field. Peer deberiefing was engaged in throughout the research process and occurred with dotoral student colleagues and members of the original study's research team.

\section{Transferability}

Transferability was ensured through "thick description" (Denzin, 2011) of participants' experiences, meanings, actions, and processes related to participants lived experiences. Detailed information regarding the context and/or background allows for the reader to decide whether similar process might be at work in their own settings and apply this study to similar populations (Merriam \& Tisdell, 2015). 


\section{Dependability}

Dependability was achieved through an audit trail, and detailed and thorough explanations of how the data was collected (Lincoln \& Guba, 1985; Merriam \& Tisdell, 2015)

\section{Confirmability}

Confirmability was achieved through an audit trail (as described above) and researcher reflexivity (Lincoln and Guba, 1985).

\section{Positionality and Reflexivity Statement}

I am a female, Caucasian, cis-gendered, German native. I was born and raised outside of the United States and have lived in this country for a decade. I am aware that being from a different country and culture has informed my worldview, which may differ from research participants. I do not share many of the demographic characteristics of my study's participants. Not being native to the U.S. has been beneficial throughout the research process in terms of participants' openness and their desire to educate me about their lived experiences as older AAPPLWHA. My engagement in the original study allowed for me to get to know participants, earn their trust and familiarize myself with the African American culture and history in the U.S., as well as the systemic racism that is still prevalent today. To prevent my own biases, I relied heavily on peer debriefing with members of the original research study.

This chapter describes the rationale for a qualitative research design and constructivist grounded theory methodology. It includes a description of the research sample, an overview of research design and the methods of data collection. Further this chapter discusses the analysis and synthesis of the data and concludes with ethical considerations and rigor, trustworthiness and concludes with a positionality and reflexivity statement. 


\section{CHAPTER FIVE: PARTICIPANT DEMOGRAPHICS AND OVERVIEW OF THE PROCESS OLDER AAPLWHA EXPERIENCE RELATED TO THEIR INCARCERATION AND ENGAGEMENT IN CARE}

The purpose of this constructivist grounded theory study was to understand the processes older African American adults experience related to their incarceration and engagement in care. This chapter presents participant demographics and a context specific framework, which is derived from the 22 in-depth interviews, seven follow up interviews and a focus group conducted with formerly incarcerated older AAPLWHAs. The conceptual model responds to the study's two primary goals:

1. To understand and provide deep description of the varied dimensions of the experiences of incarceration among older AAPLWHA

2. To develop an inductive theory of the process related to incarceration experiences among older AAPLWHA and their engagement in care.

The model conceptualizes the varied dimensions of the experience of incarceration of older AAPLWHA and captures the process related to incarceration experiences of older AAPLWHA and their engagement in care. The model also addresses the two main research aims and the corresponding sub-aims, which will be discussed in detail in Chapters $6-8$.

\section{Characteristics of Study Population}

Of the 22 study participants, 18 participants were male, three participants were female and one identified as transgender. Regarding sexual orientation, 14 of the participants identified as heterosexual, three as bisexual, three as homosexual and one 
indicated to be unsure. The original study divided participants' age into three age categories from youngest ( 50 - 60 years), middle (61 - 70 years) to oldest (over 70 years). At the time of the interviews, 55 percent of the participants were between $50-60$ years of age and $45 \%$ were between the ages of $61-70$ years. None of the participants were aged 70 and over. Seven ( 32 percent) out of the 22 participants were diagnosed with HIV while in incarcerated; three (14 percent) decided to engage in HIV testing even though they had previously received an HIV diagnosis. Of the 22 individuals, 15 (68 percent) entered the correctional system at least once as an older adult (age 50 and over). The remaining seven ( 32 percent) participants were incarcerated before age 50 but experienced living with HIV while incarcerated. One of the female participants, Midnight, was the only participant to have ever worked in the criminal justice system. Her experience was unique in the sense that she worked as a correctional officer in the criminal justice system for ten years before she was incarcerated herself. During that time she interacted with HIV positive individuals while knowing and not disclosing her own HIV status. 
Table 5.1 Participant Characteristics

\begin{tabular}{|c|c|c|c|c|c|}
\hline Name & Gender & $\begin{array}{c}\text { Sexual } \\
\text { Orientation } \\
\end{array}$ & $\begin{array}{c}\text { Age } \\
\text { Group* }\end{array}$ & $\begin{array}{c}\text { Diagnosed } \\
\text { while } \\
\text { incarcerated }\end{array}$ & $\begin{array}{c}\text { Year } \\
\text { diagnosed }\end{array}$ \\
\hline Nana & male & Bisexual & Middle & No & $1990 \mathrm{~s}$ \\
\hline Midnight & female & Heterosexual & Middle & No & 1990s \\
\hline Jimmy & male & Homosexual & Youngest & No & $1990 \mathrm{~s}$ \\
\hline Lil Booger & male & Heterosexual & Middle & No & $1980 \mathrm{~s}$ \\
\hline James & male & Heterosexual & Middle & No & $2000 s$ \\
\hline Beau & male & Heterosexual & Youngest & Yes & 1990s \\
\hline Billie & transgender & Not Sure & Youngest & No & $1990 \mathrm{~s}$ \\
\hline Zeus & male & Heterosexual & Youngest & Yes & $2000 s$ \\
\hline Red & male & Heterosexual & Middle & No & 1990s \\
\hline Gee & female & Heterosexual & Youngest & No & 2009 \\
\hline Doc & male & Heterosexual & Youngest & Yes & $2000 \mathrm{~s}$ \\
\hline Baby Bug & male & Heterosexual & Middle & No & $1990 \mathrm{~s}$ \\
\hline Gus & male & Heterosexual & Youngest & No & $2000 \mathrm{~s}$ \\
\hline Eminem & male & Homosexual & Youngest & Yes & $1990 \mathrm{~s}$ \\
\hline Free Time & male & Heterosexual & Middle & No & $1990 \mathrm{~s}$ \\
\hline 1960 & male & Heterosexual & Youngest & Yes & 1990s \\
\hline Stew & male & Heterosexual & Middle & Yes & $2000 \mathrm{~s}$ \\
\hline Jethro & male & Heterosexual & Youngest & Yes & $1990 \mathrm{~s}$ \\
\hline Rabbit & male & Bisexual & Middle & No & $1980 \mathrm{~s}$ \\
\hline Josephine & female & Bisexual & Youngest & No & $1990 \mathrm{~s}$ \\
\hline Topher & male & Homosexual & Youngest & No & $1980 \mathrm{~s}$ \\
\hline Peter & male & Homosexual & Middle & No & $1980 \mathrm{~s}$ \\
\hline
\end{tabular}

Age group* Youngest (50 -60 years), Middle (61-70 years), Oldest ( $>70$ years) 
Table 5.2 Participant Demographics

\begin{tabular}{lcc}
\hline \multicolumn{1}{c}{ Demographic } & Number & Percent \\
\hline Age (N=22) & 12 & 55 \\
$50-60$ & 10 & 45 \\
$61-70$ & 0 & 0 \\
Over 70 & & \\
Gender & 3 & 13 \\
Female & 18 & 82 \\
Male & 1 & 5 \\
Transgender & & \\
Sexual Orientation & 14 & 64 \\
Heterosexual & 4 & 17 \\
Homosexual & 3 & 14 \\
Bisexual & 1 & 5 \\
Not sure & & 5 \\
Marital Status & 1 & 55 \\
Married & 12 & 9 \\
Single, never married & 2 & 13 \\
Widowed & 3 & 9 \\
Divorced & 2 & 9 \\
Domestic partnership & 2 & 32 \\
Other & & 68 \\
Diagnosed while incarcerated & 7 & 18 \\
Yes & 15 & 53 \\
No & & 27 \\
Year diagnosed & 4 & \\
1980s & 12 & \\
1990s & 6 & \\
2000s & & \\
\hline
\end{tabular}

Overview of the Process Older AAPLWHA Experience Related to Their

\section{Incarceration and Engagement in Care}

The model in Figure 5.1 describes older AAPLWHAs incarceration experiences in four phases. Transitional processes capture the varied dimensions, which become evident through the behavioral and psychological processes individuals engaged in. 


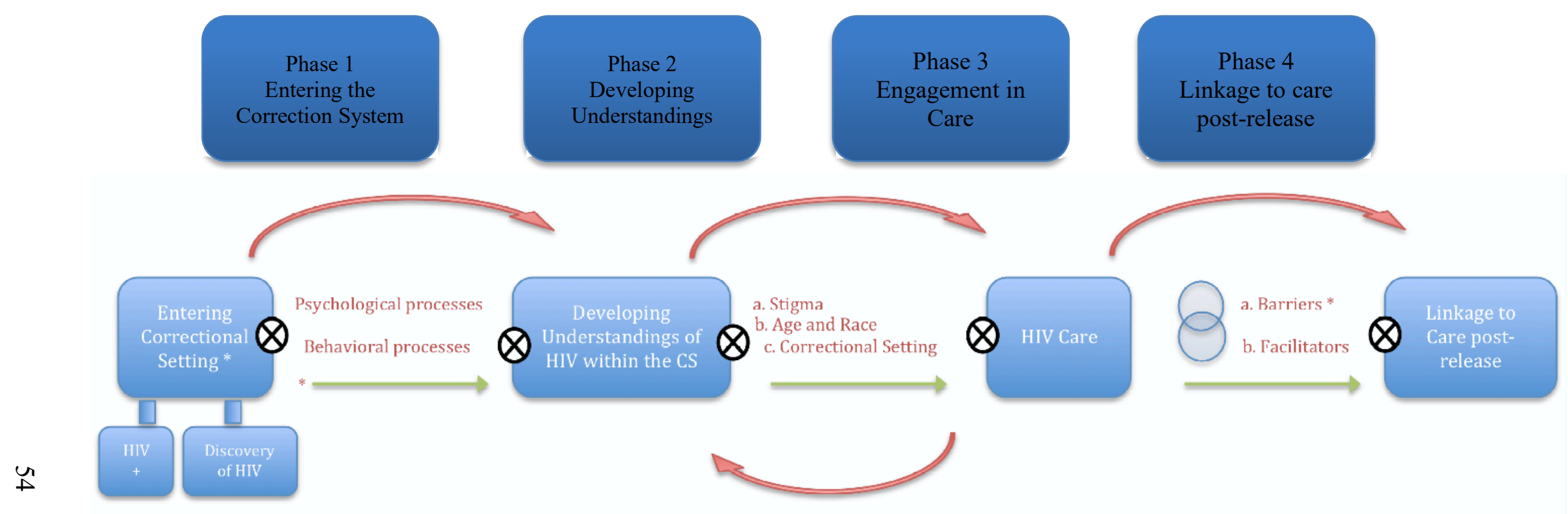

$\longrightarrow$ Pathway through which the incarceration is experienced

* As older ( $>50)$ AA adult; $\mathrm{N}=15$

Overlap between Barriers and Facilitators

Transition

$$
\begin{aligned}
& \text { * Barriers } \\
& \text { - Confidentiality and } \\
& \text { disclosure } \\
& \text { - Experiencing discrimination } \\
& \text { - } \text { Ttime } \\
& \text { * Facilitators } \\
& \text { - Benefitting from access to care } \\
& \text { - Stabilizing effect on health } \\
& \text { - Social support } \\
& \text { - Special rules for HIV+ inmates }
\end{aligned}
$$


Figure 5.1 Overview of the Process older AAPLWHA experience related to their Incarceration and Engagement in Care 


\section{Phase 1: Entering the Correctional Setting}

In phase 1 individuals entered the Correctional System. The context specific framework accounts for the difference of being diagnosed with HIV prior to incarceration and individuals who discover their HIV diagnosis while in the correctional system. In addition, depending on the pathway through which incarceration is experienced (i.e. jail, state or federal prison) the process of entering the correctional system varied for participants. While some participants experienced more than one incarceration setting over their lifetime, other participants' incarceration experience was a single event and took place in a jail. In jail, HIV testing was non- existent and the resources for HIV care engagement were sparse. As individuals transitioned from the first to the second phase, they began to engage in psychological and behavioral processes. Behavioral and psychological processes were instrumental contributing to the development of understandings of HIV within the correctional setting.

\section{Phase 2: Developing Understandings}

Phase 2 constitutes the development of understandings of HIV within the correctional setting. As individuals transitioned from the first to the second phase, they began to engage in psychological and behavioral processes. These processes appeared differently depending on whether individuals were HIV positive prior to incarceration, or were first diagnosed while incarcerated. Psychological processes included the adaptation to being institutionalized and transitioning from their daily lives on the outside into the correctional setting. The loss of freedom and autonomy individuals described influenced those processes throughout participants' incarceration experiences. Behavioral processes captured the actions of older AAs as they navigated the first two phases and throughout the incarceration experience. Participants who were aware of their HIV status prior to incarceration displayed two distinct behavioral strategies. While some individuals 
actively advocated for their needs concerning their HIV care, others "laid low" hoping to avoid discrimination and stigma. Gee, recalled her experience of being processed at the local jail utilizing the first strategy. She was proactive and advocated for herself in terms of accessing her HIV medication.

What it came down to was that I needed to make a phone call. One of my housemates was able to get my drugs down to the police station. I told the officer I have to take my medication. They sure don't want anyone dying on their watch they weren't compassionate you know it was to protect themselves.

Using the second strategy, another study participant, Doc, "laid low" hoping to avoid discrimination and stigma from other inmates and correctional officers:

Nobody can know that you are HIV I was trying to keep that stuff secret but it is hard if you have to get in a long line everyday to get your medicine. I also hid my meds in my drawer so my cellmate doesn't know I am taking them, again no one can know!

Participants who were diagnosed while incarcerated were faced with an additional layer of complexity regarding strategies for meeting their needs around HIV care. Not only did they have to conceptualize what it means to live with HIV, but also quickly adapt to what it meant to live with HIV within the correctional system. James who was diagnosed while incarcerated shared how he learned about his HIV diagnosis:

They called me in the office, and they said that they had some bad news for me. I said, "What is the bad news?" They said "You have been infected with the HIV virus and you will die if you don't start taking the medication. Then they just send me back to my cell and told me I would just see the doctor from time to time.

That's it. Now I was HIV and back alone in my cell.

Like many participants who were diagnosed while incarcerated, James received little to no psychological support or education concerning his diagnosis. Consequently, the development of understandings of HIV within the correctional setting occurred simultaneously while making sense of his HIV diagnosis. He shared, "From this day I was HIV positive and I didn't know what that means for me now and then I was fresh incarcerated on top of it you know that was just overwhelming." 
Whether learning of their HIV status prior to or after incarceration, understandings of how to navigate living with HIV within the correctional setting required evolving strategies throughout the incarceration experience. Participants engaged in a variety of behavioral and psychological processes that were dependent on their environment (jail or prison), access to medical care and a variety of other influencing factors. The transition from the second to the third phase was often characterized by the influence of stigma (internal and external stigma), the respective correctional setting and how age and race impacted ones' experience.

\section{Phase 3: Engagement in Care}

The third phase constitutes engagement in care. As individuals enter this phase they have developed initial understandings of HIV within the correctional system, and for many, engaged in strategies to receive support, or maintain confidentiality. Those understandings were broad and differed among individuals. Beau, for example, spoke about his realization that he was able to access HIV care while incarcerated that would have not been possible outside the correctional system due to his lack of insurance. He described, "You know I was able to get my HIV medicine in here for free. Out there I would not have taken it you know it is just so expensive." Eminem's initial understandings of HIV within the correctional system were shaped by fears of discrimination and stigma. Consequently, participants' initial understandings set the stage for either engagement or disengagement in care. Initial understandings differed based on the respective correctional setting. Overall, participants who were incarcerated in a jail characterized a lack of access to HIV care and an overall situation that was marked by uncertainty and constant change. In contrast, participants who were in a prison expressed a longer-term perspective with understandings (i.e. HIV care) built with a long-term perspective in mind. 
Additionally, in phase three, participants built on those understandings as they navigated the health care system. Participants discussed barriers and facilitators to care, a topic which will be discussed in detail in the following chapters.

\section{Phase 4: Linkage to Care}

The fourth and final phase constituted linkage to care post release. Older AAPLWHA described their experiences as they left the correctional system and transitioned back into a community setting. This phase was characterized by overwhelming barriers such as the loss of access to health care, challenges in securing housing, a lack of resources and personal agency to coordinate their own HIV care, or locate and qualify for resources. Few participants described a smooth transition leading to continued engagement in HIV care upon release. Participants emphasized that in order to successfully be linked to care, access to health insurance was instrumental, but often not available after leaving the correctional setting. Upon release, individuals left the physical incarceration behind but also left behind their access to HIV care. Disengagement of HIV care often resulted.

The processes older AAPLWHA experienced related to their incarceration and care engagement were multifactorial. These processes accounted for the time of incarceration (ranging from the early 1980s - 2015), the correctional setting, prior HIV diagnosis, or HIV discovery while incarcerated, and being diagnosed pre and post HAART (highly active antiretroviral therapy), all of which impacted the medication regiment received. In the case of multiple incarcerations, the process was iterative in the sense that participants moved through the phases multiple times yet often via different correctional settings. These transitions added different layers and complexity to their experiences and the access to continued engagement in care. In addition, the transition between the phases was different within each incarceration experience. For example, 
during a second incarceration experience, a participant had already developed an understanding of what it meant to live with HIV in the correctional setting, and built on this experience to use a different strategy when he or she faced incarceration again. Zeus, who had multiple incarcerations stated, "I knew what to expect when I went in (prison). My first time I disclosed (meaning HIV status) and that was a mistake. The second time I learned my lessons and kept my cards close."

In this chapter I have provided a description of the study population's demographic characteristics and introduced the processes older AAPLWHA experience related to their incarceration and engagement in care. The process is described in the four phases, with transitions depicted in Figure 5.1. Next, I present findings related to Aim 1 of how older AAPLWHA draw meaning from their incarceration experiences. 


\section{CHAPTER SIX: HOW DO OLDER AAPLWHA DRAW MEANING FROM THEIR INCARCERATION EXPERIENCES?}

Meaning making was a critical component for older AAPLWHA as they tried to make sense of their incarceration experiences, both interpersonally and within their environment. Experiences related to incarceration impacted participants on multiple levels, some immediate understandings and some that evolved over time. During the data analysis three main factors emerged from participants' accounts and contributed to their ability to draw meaning from their incarceration experiences. First was the influence of stigma (HIV and incarceration stigma), second, the different pathways through which the incarceration is experienced, and third involved age and race. Once released, the experiences of incarceration also had consequences for older AAPLWHA regarding their relationships with the outside world (family and communities).

This chapter presents findings corresponding to the first research aim of how older AAPLWHA draw meaning from their incarceration experiences in terms the (a) influence of stigma (HIV and incarceration stigma), (b) the different pathways through which incarceration is experienced, and (c) how age and race impact one's incarceration experience. I will now present findings on each in turn, organized in terms of category, properties and dimensions. 
How do older AAPLWHA draw meaning from their incarceration experiences?

\section{Sub-Question: Explore the Influence of HIV Stigma}

This sub-question is answered by introducing two figures. The first section is about HIV stigma (see Figure 6.1) and the second figure addresses incarceration stigma (see Figure 6.2).

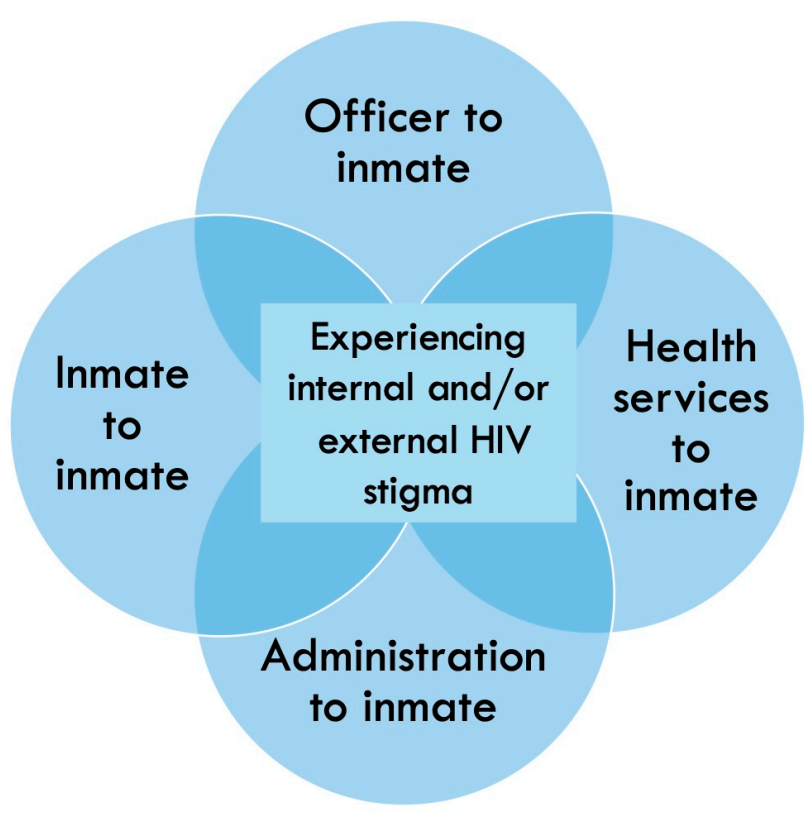

Figure 6.1 Experiencing Internal and/or External HIV Stigma

Exploring the influence of HIV stigma in terms of meaning making made evident that participants experienced two kinds of HIV stigma: internal and/or external HIV stigma. Internal HIV stigma was often self-imposed and characterized by anticipating and fearing discrimination. External HIV stigma was characterized through the experience of unjust treatment, such as being in segregated housing (due to HIV status) or being prohibited from working in certain areas such as the kitchen. The following four realms emerged from the analysis: (1) experiencing stigma via inmate to inmate, (2) experiencing stigma via correctional officer to inmate, (3) experiencing stigma via health services and (4) experiencing stigma via facility administration. The internal and/or 
external stigma that participants experienced within each property ranged from subtle to overt. The distinction between subtle and overt was not all or nothing but nuanced, depending on individuals' unique experience and the specific context. Overt internal and/or external stigma was characterized through one or more of the following: experiencing discrimination, being physically mistreated and/or abused, being isolated (i.e. having to live in segregated housing), losing privileges (i.e. participating in activities with the general prison population), or receiving judgmental statements and avoidance behavior (i.e. correctional officers, health care workers or other inmates keeping their distance). Subtle internal and/or external stigma was perceived to exist more on a structural level, particularly in regards to a lack of education about HIV. Insufficient resources for older AAPLWHA, such as support groups, perpetuated an atmosphere where subtle stigma thrived. Subtle expressions of stigma also consisted of insensitive and/or demeaning remarks from others, such as health care professionals, who negated older AAPLWHAs perception of reality. The subtle conveyance of stigmatizing messages by others became a barrier for participants and impacted their incarceration experience in a negative way. Participants highlighted the lack of support groups for PLWHA in the correctional system, and a lack of HIV education among other incarcerated individuals, correctional officers, the facility administration and even health care professionals.

\section{Experiencing Internal and/or External Stigma from Inmate to Inmate}

Experiencing internal and/or external stigma from inmate to inmate was a common experience and concern for participants. Jethro elaborated on the experience of how other inmates stigmatizing attitudes caused him to experience increased internal stigma: 
If they [other inmates] referred to it as the ninja it made me feel like I am dirty, you know. Like there is something wrong and dirty inside me. That's an awful feeling.

Similarly, Doc described what it was like for him in regards to overt external and internal stigma from other inmates. He described:

First of all nobody can know because everybody is gonna treat you like an outcast. I have seen the stigma. I have felt it. I've seen it and its bad. In prison they're ready to kill you if you're in there. And not just that it also makes you feel bad that you're carrying this disease inside.

Even though Doc had not been personally physically harmed or threatened, he experienced overt internal and external stigma during the time of his incarceration. The experience of HIV stigma from inmate to inmate was perpetuated by the nickname "the ninja" by which incarcerated individuals referred to the HIV virus. Several participants discussed their experience and elaborated on how this association increased the internal and external HIV stigma they perceived from other inmates. In Jimmy's case, learning what it meant to have "the ninja" added to the external stigma he already felt from other inmates. He remembered:

Well it was very difficult because the people there that I was incarcerated with weren't educated concerning HIV. They gave it a nickname they called it the Ninja and I didn't know what that was, they said "Oh, he has the ninja". Never had heard it before in my life. I'm like ok, what's the Ninja? They had to educate me. It made me more uncomfortable there was a lot of stigma for sure.

Thus for Jimmy, learning that HIV is referred to as "having the ninja" shaped his experience and increased the internal stigma surrounding HIV. This experience also illustrates how Jimmy strove to create an understanding of what it meant to live with HIV while incarcerated. He explained "having HIV is different when you are incarcerated you know there is more stigma attached to it and the other inmates called it the ninja and not HIV. All of that made me feel dirty how others are so afraid of getting my disease that they call it that." Midnight contributed a unique perspective regarding the internal and external HIV stigma between inmates. As described in Chapter 5, she worked as a 
correctional officer prior to her own incarceration. When asked about the HIV stigma between inmates Midnight recalled, "I've heard a lot of the prisoners like, man, I don't wanna go over there to dorm 10. All them inmates, half of them got AIDS and stuff like that. I was never gonna disclose myself when I was incarcerated after hearing stuff like that." Remembering such stigmatizing messages during her incarceration and the fear of being stigmatized herself kept Midnight from disclosing her HIV status and consequently prevented her from receiving HIV care. Pervasive stigma among inmates was a significant finding that overshadowed participants incarceration experiences.

\section{Experiencing Stigma from Officer to Inmate}

Experiencing internal and external HIV stigma from officer to inmate was prevalent among this study's participants. Billie elaborated on her experience of external overt stigma coming from correctional officers, "I noticed some officers were discriminating us. They would not want to go in your cell because you were HIV and they would spread your business [HIV status] around." Likewise, other participants also mentioned the overt stigma experienced through correctional officers. Jimmy described:

I noticed some staff I felt they were kind of discriminatory. When I first got there [meaning the correctional facility] I had to carry my own bags. Can you imagine? Me being, I weighed about 165 pounds. I lost a lot of weight. I couldn't even carry my own bags. I'm going up steps, my bag busted off, my personal stuff was out there. The guards just stood there and watched me. They wouldn't help me or nothing. It was the inmates that came out and helped me put my stuff in the bag and all that. I knew right then I wasn't going to get no special. They were treating me kind of bad. They kind of talk down to you. I didn't like that.

Besides overt stigma participants also experienced subtle expressions of external and internal HIV stigma. For Gee, for example shared, "sensing the stigma" was generated through an interaction with a correctional officer. She recalled:

When you are being processed and you have the corrections officer knowing why you there and sensing the stigma. You get checked in, they take your belongings. I had to take a shower and then I was finally put into a jail cell so that wasn't a very pleasant situation. I just felt like I failed as a human being. 
A pervasive theme was a lack of education on the side of the correctional officers that led to overt and subtle HIV stigma. Doc's experience below speaks to the apparent lack of education that was prevalent among the correctional officers:

Some correctional officers didn't want to deal with me because they were literally ignorant on the deal. They scared if they hand me the tray, that they'll catch it. I could tell. They'd ask to get transferred. They don't want to work if they got to deal with HIV. I went through that. It is terrible.

An exception to this finding was present among participants who were on a medical unit or had been given special privileges such as a single cell. They reported less stigma from correctional officers, and recalled more positive interactions. Stew, who spent much time of his incarceration time on a medical ward and single cell described:

It was okay and the guards were great for the most part because during that time I was on oxygen, they kept my machine for the oxygen on the outside of the door and I had a single cell so I was by myself like I was on my own little pod. They ran the cord under the door and plus I had a little TV. It was okay you know most people wouldn't tell you that.

However, participants' disclosure of positive interactions was limited. Overall, the lack of education concerning HIV among correctional officers was prevalent throughout participants' experiences. Interactions with correctional officers were overshadowed by a pervasive lack of education that resulted in overt and subtle stigmatizing messages.

\section{Experiencing Stigma from Health Services to Inmate}

Another area, in which participants experienced internal and external HIV stigma, was from health care providers. Interactions with health care providers were vital to access HIV care and medication. Yet, the majority of older AAPLWHA reported concerns regarding the stigmatizing messages that they received from health care workers, which ranged from subtle to overt. Doc recalled an interaction he had with a nurse, "this one nurse put on two pairs of gloves because she was scared of me and she had an officer come in the room to protect her I know why she did that HIV is why she did 
that." Doc witnessed the nurse's overt stigma towards him and internalized the stigmatizing message. He continued, "That made me feel worse. They think you are dirty and I felt it myself." Zeus described a similar, but more subtle life threatening experience about delays in his care. He shared, "I know that all these rules and regulations has to do with that they don't want us to get care. The nurses take their time and you can die in there [prison] they ain't in no hurry to get you nothing. That made me think I am nothing like I am not important." Overall, interactions with health care providers outside of the correctional setting were perceived as more positive than those with health care professionals that were located in the correctional setting. 1960 spoke to this experience, "The nurses and doctors in the facilities were treating you differently than the ones outside. I felt more stigma from them [health care providers inside the correctional system]." Additionally, in some correctional settings the HIV care was delivered remotely via a screen. Most of the participants experienced this mode of care delivery as distant, less personalized and lacking confidentiality. Eminem elaborated, "they had this screen where you could see the doctor but you never knew when you would see him and it was not confidential. There was the correctional officers right with room in the room listening to everything. You can feel the stigma." Jethro shared, "You know how can they [health care providers] look at you through a screen and examine you. "I could tell that he [doctor] didn't really care about my health it was like asking some questions and 5 minutes later it was done." Jethro's experience illustrates the overt external stigma that he felt from this experience relative to health care providers.

\section{Experiencing Stigma from Administration to Inmate}

Participants frequently reported both overt and subtle examples of enacted stigma from members of the administrative staff. Overt external and internal stigma was most 
clear in policies that impacted participants on a daily basis, such as segregated housing or not being permitted to work in the kitchen. Billie stated:

The administration does not care about the people with HIV the only thing they're there for is the protection of the penitentiaries. When they find out you are HIV they have a change in the way they treat the person with HIV and just like that you have to live in a different dorm. How do they think this makes you feel? I felt I there is something really wrong with and I am a danger to others

Concerns about disclosing one's HIV status were common; participants shared the fear that the prison administration would not maintain their confidentiality. Jimmy noted:

There is nothing as an inmate you can really do about the stigma from above [meaning the prison administration]. They do let information slip to the officers you know. They were in your face, show you respect, make you think that they're doing their job and they're not going to do anything to put your business out there. As soon as you turn your back in the office, you know they tell everyone he's got $H I V$.

Subtle stigma occurred more on a structural level and became evident through the lack of resources and flexibility on the side of the administration. Participants voiced the need for more education concerning HIV, especially for officers and inmates to reduce the stigma. Eminem shared, "We needed them to educated prisoners about HIV how you can catch and how not, and not only them the guards need education too." This apparent lack of education perpetuated an atmosphere where subtle stigma thrived.

The influence of stigma on the incarceration experience was further complicated by incarceration stigma. Exploring the influence of incarceration stigma in terms of meaning making suggested that participants experienced two kinds of incarceration stigma: anticipated (e.g. expectations of being stigmatized and/or rejected) and enacted (actual stigmatizing experience, being rejected or discriminated against) incarceration stigma. Additionally, being older and having a criminal record were also identified as significant properties. All four realms were of concern for participants as they were involved in the criminal justice system. 
How do older AAPLWHA draw meaning from their incarceration experiences?

Sub-Question: Explore the Influence of Incarceration Stigma

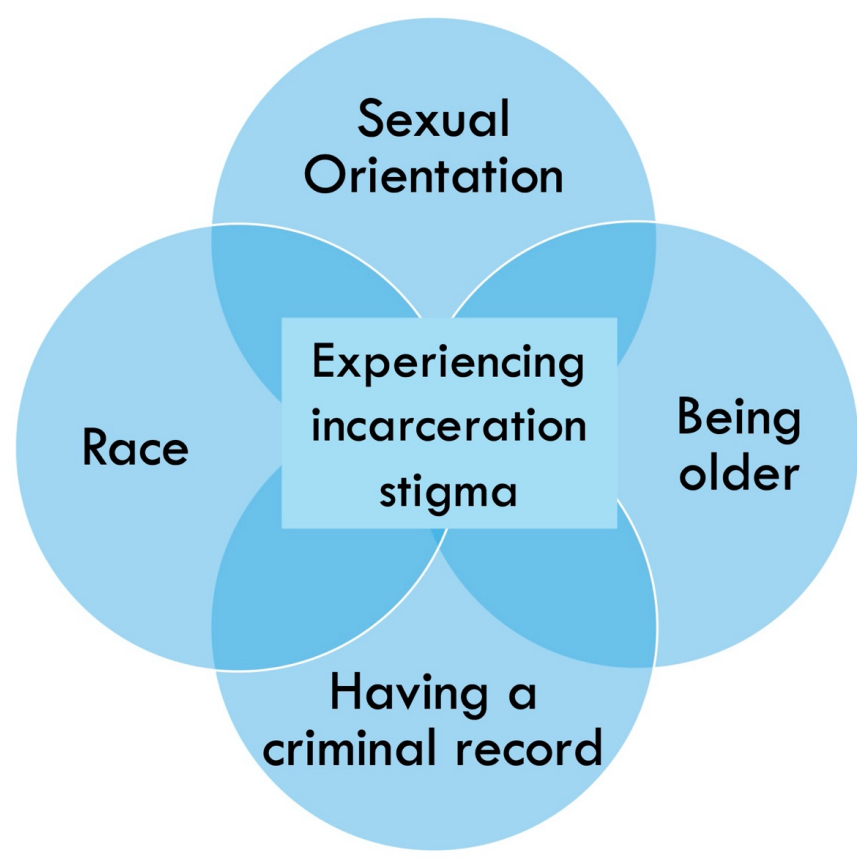

Figure 6.2 Experiencing Incarceration Stigma

\section{Experiencing Incarceration Stigma in terms of Race Within and Outside of the}

\section{Correctional Setting}

Incarceration stigma in terms of race not only influenced the incarceration experience, but also added to the challenges that AA participants faced in their own communities post-release.

\section{Within the Correctional Setting}

Participants expressed their experiences of incarceration stigma in terms of race as anticipated and/or enacted. Billie explained how she experienced incarceration stigma within the correctional setting. She stated, "comparing to a Caucasian person in prison you are already different and not allowed to join a lot of gangs and groups and stuff. That's the way it is for us being Black in prison." Zeus depicted his experience of being Black and incarcerated: 
They sending Blacks to prison to help make these concentration camps. Most of our prisons are now privately owned. If they fill them up and put a lot of Blacks in it them they are making money...I am an American and because I was incarcerated I am treated like I am not. I am a felon. They say you were an inmate you have no rights.

Similarly, Doc described incarceration stigma and race from his point of view " $I$ am telling you it's already bad if you are Black and male. When you are Black and in prison your done." For some participants, being subject to the stereotype of being Black and incarcerated was most distressing. For other participants, the incarceration stigma they faced within their own communities was worse.

\section{Outside of the Correctional Setting}

Billie's story continues to illustrate the incarceration stigma she faced post release "Being Black in a Black community and knowing Black nurses working in prison and I know what Black nurses do in prison. They do break the confidentiality and that you have been to prison gets back to your community. So once you released you really have problems!" Jimmy recalled his concerns post release. He described, "I was worried no one will hire me because I am Black and a felon and have a record. And that's not all. I was treated different in my community too. ” As his experience illustrates, anticipated stigma followed participants' post-release and impacted their ability to reintegrate into their communities. Stew elaborated on experiencing enacted stigma. He shared, "I was treated different because of my incarceration. I had that reputation and it doesn't go away it follows you." Incarceration stigma post release was distressing for participants, especially as they tried to reestablish their lives outside of the correctional system.

\section{Experiencing Incarceration Stigma in terms of Sexual Orientation Within and}

\section{Outside of the Correctional Setting}

The link between sexual orientation and incarceration stigma was prevalent among participants. One's sexual orientation often exacerbated the incarceration stigma. 
It became evident that incarceration stigma in terms of sexual orientation was experienced within and outside of the correctional setting.

\section{Within the Correctional Setting}

Participants who identified as homosexual described the pervasiveness of "homophobia" within jail and prison. Heterosexual participants also explained that they were often presumed to be gay based on their HIV diagnosis and incarceration history. Rabbit brought up that as an inmate who is HIV positive one is automatically perceived as having had sex with another man. Rabbit described, "Everyone is ignorant in that deal. If you've got HIV and you are in prison then you have to have sex with a man. That's the way they [inmates and correctional officers] see it." Baby Bug, a heterosexual participant recalled the enacted stigma he observed towards gay, HIV positive inmates:

People in prisons in Kentucky don't really get the care that they need. I mean HIV positive people, but really the ones that are gay. I don't care what they do but they should start giving them the care and give out condoms.

Another example illustrating enacted stigma was brought up by Stew, a heterosexual participant. When asked about stigma in terms of incarceration and sexual orientation, he recalled a conversation that he had with his mother while being incarcerated, "She [mother] asked me if I am still normal what she meant to ask is if I am messing around with men. She was thinking I was with another man because I was in prison."

\section{Outside of the Correctional Setting}

Stew's conversation with his mother reflects a common experience that participants mentioned. Once they were released, their families and communities often assumed they were homosexual or engaged in homosexual experiences. For some participants this was particularly distressing, while others did not seem to be affected as much. Doc for example, expressed his concerns, "You know they look at you when you 
get out and they think you are gay now. That you have done things with other men while you were incarcerated. For them that is a fact." Billie explained, "for me it was different I am transgender I was in segregated housing from the start but I know for a lot of people there is a lot of stigma when it comes to sexuality and sexual orientation. Especially for them straight guys it is not easy once they get released." Gus, a heterosexual participant described, "I did have a hard time with the stigma once I got released. I felt that the folks in my community but more my family were looking at me differently than before. It is hard to live with that once your out of there." Incarceration stigma in terms of sexual orientation was of concern for participants within and outside of the correctional setting.

\section{Experiencing Incarceration Stigma in terms of Being Older}

A common experience for participants was the experience of incarceration stigma in terms of being older. Older AAPLWHA elaborated on how their age affected their incarceration experiences in different ways. Some participants claimed that being older served to their advantage. They described less incarceration stigma and special protections such as living in a dorm for older adults, better access to medical care, and other special accommodations not granted to younger inmates. Doc recalled, "they made sure I was on the lower bunk. I did have privileges for being a little older. I was helped more than the younger inmates."

In contrast to Doc's experience, other participants talked about how being older and incarcerated led them to experience more stigmatization. They described difficulties receiving medical care and special accommodations, such as supplemental nutrition. This occurred to Jimmy when he expressed his need for an additional snack to a correctional officer. He shared, "I told them I am older and I need to eat with those meds that I can't go that long without having food but they really didn't care about that. They was thinking probably he is a criminal why should he get special treatment." For Jimmy, this 
experience reinforced his belief that his needs don't matter and that he is not given special privileges due to his age.

Participants were confronted with how being incarcerated at an older age had impacted them physically and accelerated their aging process. Zeus recalled how he experienced his incarceration at an older age:

I was in my 50s when I was incarcerated my hair turned gray and my body got weaker. You realize when you at a certain age some things you can't do you in prison you have to bunk and that is hard when you're older.

Zeus is one of many participants whose experiences with aging while incarcerated demonstrated stigma attached to his physical and mental needs as an older AAPLWHA.

\section{Experiencing Incarceration Stigma in terms of Having a Criminal Record}

As older AAPLWHA transitioned back into their communities, their criminal record often had an adverse impact on them. The in-vivo code "you don't have no rights" was fitting to capture participants' experiences. All older AAPLWHA faced hardships in securing housing and employment during their reentry process. Additionally, they encountered a myriad of other barriers related to their incarceration history. Most participants experienced rejection by family and friends as well as stigma and/or harassment within their communities. Gus talked about the difficulties in securing housing after being released from prison. He said "that [having a criminal record] made it really hard for me to stay somewhere I ended up not finding a place to stay because no one really wants a criminal staying at their place." Similarly, Beau articulated the difficulties of having a criminal record and what the label of having been incarcerated meant to him:

They take that prison label and tell you that you were an inmate. You don't have rights. I have seen it so much but I try not to live in discrimination because it is all around me. I am an American but I am treated like I'm not. I am a felon. 
Having a criminal record post-release was one area where older AAPLWHA felt the incarceration stigma stronger than the stigma of having HIV. Participants discussed how they were not required to disclose their HIV status to potential employers, but they were required to disclose their criminal record. In this situation, a criminal record was a more salient issue than older age or HIV status. James's experience illustrates this finding. He explains, "You don't need to tell no employer about your HIV but they sure know that you was in prison you know."

Overall, incarceration stigma was a crucial component of older AAPLWHAs incarceration experience and the stigma most impacted their post-release experiences.

Next, I will describe the second sub-aim of the first research aim regarding the different pathways through which incarceration was experienced.

How do older AAPLWHA draw meaning from their incarceration experiences?

Sub-Question: Explore the Different Pathways through which the Incarceration was

\section{Experienced}

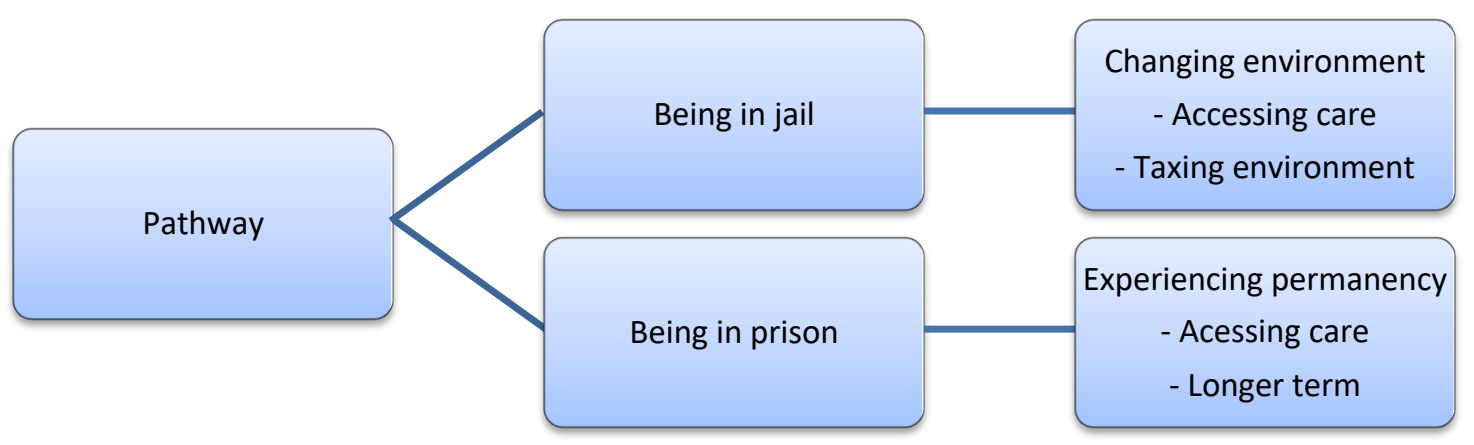

Figure 6.3 Pathways through which the Incarceration was experienced

Exploring the different pathways through which incarceration was experienced in terms of meaning making resulted in two major pathways: jail and prison. Being in jail was characterized by a changing environment involving two dimensions: (a) accessing 
care and (b) a taxing environment were identified. Being in prison was characterized through the property experiencing permanency involving one similar and one different dimension: (a) accessing care and (b) being of longer-term duration.

\section{Being in Jail}

Being in jail was overshadowed by a constantly changing environment. Given the short-term nature of this type of incarceration, the two in-vivo codes "where's my meds at?" and "living with an end in sight" illustrate participants' experiences. Most participants were unable to access their medication while they were incarcerated in a jail. For the majority of individuals, determining the cost of persistence in trying to access medications led to the conclusion that it wasn't worth the struggle since their incarceration was relatively short in time with the "end in sight." Jospehine described the situation as, "I wasn't going to be in there more than two days so I thought myself I just skip my meds that will be less trouble for me."

Lack of privacy was another issue in jails given that the incarcerated population changed daily; most participants recalled shared a large cell with bunks sometimes for weeks at a time. Participant Midnight who was incarcerated in a jail for a week explained, "there was different people everyday and they [correctional officers] didn't give me anything [HIV medication] then I went and asked and they couldn't do it because they had just too much going on in there on top of it I was pregnant and I knew how important it was for me to take my medications because it was gonna help so that my son doesn't end up with it." Midnight's excerpt describes the lack of access to care that was pervasive among participants housed in jail. Another participant, Nana, was told that he would have to stay in jail for 12 hours, but he ended up not being processed for several days and was without his HIV medication during that time. Nana explained:

I was supposed to be in lockup for 24 hours but it took them longer to process me...they was lying to me about getting out and I am in my street clothes, sleeping 
inside here with 60 other guys and no bunks on the floor. That was the worst time. I didn't get a chance to ask about my medicine because according to the officer I would be in there 12 hours max. 9 to 5 didn't happen. They didn't give a heck medicine or not. Shut up and wait that is basically what they tell you.

Based on participant interviews individuals in jail were denied lifesaving medications and stalled when they asked to access their HIV medication. Zeus described his experience, "I asked for my medicine many times I need it to live it is like when you don't give insulin to a diabetic. They kept delaying it and in the end I never got it." This scenario was common for participants who were incarcerated in a jail. Gee talked about the physically taxing environment she encountered while she was in a jail. She reported, "the living conditions in jail are not very pleasant, and you know I got a taste of what it is like to be a in jail with HIV. They don't care, all they're there is to keep you locked up for your crime. So many people there in a small place and the girl next to me came off of heroin it was stressful."

Only one participant described being able to access HIV care while he was in jail. Jimmy stated:

I had access to the doctor. At the time I was going to a clinic. They was driving me from the jail in handcuffs and brought me to see the doctor. And they gave me Boost (nutritional shake) at the jail.

Overall, participants relayed experiencing more barriers in the jail setting to access care, especially when it came to HIV medications. These findings were based on the shorter duration of stay and the fact that jails lack resources and are not equipped to provide longer term and/or consistent medical care for chronic illnesses such as HIV.

\section{Being in Prison}

The in vivo code "not getting out of here soon, this is my home" captures older AAPLWHAs experiences of being in prison. Overall, being in prison was characterized by the notion of more permanency than that of being in a jail. Not only did participants 
spend a longer amount of time in prison, but they also reported increased access to care.

Gee elaborated on the difference between prison and jail. She stated:

I think in prison they're more understanding because they're there for a longer much time than they are if they're in just a jail or a county or city jail. I think the people might...the prison or federal or state jails they probably have a little bit more compassion, not much more, but a little bit more because of that situation that they're going to be there for longer.

Several participants mentioned getting connected to HIV care for the first time while in prison. Individuals elaborated on the benefits they experienced, such as being able to access care without the bureaucratic and financial barriers they faced on the outside. Beau, who was diagnosed with HIV while incarcerated reflected on his experience. He said, "being diagnosed in prison was a positive thing. I didn't have to deal with my family right away and I got the medicine I could not afford on the outside." Other participants were diagnosed with HIV prior to incarceration but had never sought or disengaged in HIV care. Many of them decided to reengage in HIV care during their time in prison. Participant 1960, who was previously diagnosed but was not taking his medication at the time of his incarceration stated, "I was already here [prison] so why not take the test again so I did and then they told me I was HIV. I already knew but I started taking them medicines. They were free and they gave it to me everyday I never missed." 1960's example illustrates how incarceration in a prison was a catalyst for reengagement in HIV care.

Next, I will describe the third sub-aim regarding how age and race impacted one's incarceration experience. 
How do older AAPLWHA draw meaning from their incarceration experiences?

Sub-Question: Explore How Age and Race Impacted One's Incarceration

\section{Experience}

Table 6.1 Impact of Age and Race on the Incarceration Experience

\begin{tabular}{|c|c|c|}
\hline \multirow{4}{*}{\begin{tabular}{l}
\multicolumn{1}{c}{ Sub-question } \\
Explore how age \\
and race impacted \\
one's incarceration \\
experience
\end{tabular}} & Category & Properties \\
\hline & $\begin{array}{l}\text { Aging as HIV+ } \\
\text { AA while } \\
\text { incarcerated }\end{array}$ & $\begin{array}{l}\text { Experiencing accelerated aging } \\
\text { - Facing chronic illness and disability } \\
\text { - Living in environment designed for } \\
\text { younger individuals }\end{array}$ \\
\hline & & $\begin{array}{l}\text { "Dying anyday" } \\
\text { - Experiencing depression and } \\
\text { depressive symptoms } \\
\text { - Shifting/changing priorities }\end{array}$ \\
\hline & & $\begin{array}{l}\text { Experiencing ageism and structural racism } \\
\text { - Substandard medical care } \\
\text { - Tensions with younger inmates }\end{array}$ \\
\hline
\end{tabular}

Exploring how age and race impacted older AAPLWHAs incarceration experience resulted in the category aging as incarcerated HIV positive AA. Three properties emerged from the analysis (1) experiencing accelerated aging (2) "dying anyday", and (3) experiencing ageism and structural racism. Experiencing accelerated aging was characterized by (a) facing chronic illness and disability and (b) living in an environment designed for younger individuals. "Dying anyday" was characterized by (a) experiencing depression and depressive symptoms and (b) shifting/changing priorities. Experiencing ageism and structural racism was characterized through (a) living with substandard medical care and (b) tensions with younger inmates. 


\section{Experiencing Accelerated Aging}

The first property to explore is that older AAPLWHA experienced accelerated aging while they were incarcerated. Participants faced an array of age-related chronic illnesses and disabilities besides having HIV. Doc recalled, "I caught pneumonia every now and then... as far as that. Then I got mad depressed. That's what just wears me out." Another issue participants encountered was that the prison environment is designed for younger individuals. Prisons are ill equipped to address the needs of older individuals in general, let alone older adults living with HIV. Jimmy described, "you know my hair grayed. I came out [meaning prison] with all this grey. It is hard in prison at a certain age. Prisons are not made for older folks like us." Midnight, whose unique background as a correctional officer was introduced in Chapter 5, reflected on her experience as correctional officer observing older AAPLWHA. She explained:

He was so old he got to the point where he couldn't hear and then he started losing his sight. He also lost a lot of weight couldn't walk and stuff like that. They had to push him in a wheelchair. I think he would have been better off on the outside. I mean his HIV wasn't what made him like that it was the aging part you know.

The excerpts above illustrate how older AAPLWHA face chronic illness and disability due to the accelerated aging process. Further, participants discussed that prisons are designed to house a younger prison population and therefore, not adequately equipped to meet the needs of aging inmates.

\section{"Dying Anyday"}

The second property that emerged from the analysis was "dying anyday" and encompassed experiencing depression and depressive symptoms as well as shifting/changing priorities. The experience of depression and depressive symptoms was pervasive among the study's participants. Older AAPLWHA were careful to note that being older and incarcerated was nothing to look forward to, but rather a dooming 
experience. Jimmy elaborated, “if you're getting older and you're in prison ain't too much to look forward to you know you are at an age where you could die anyday." Likewise, Nana explained:

They [older AAPLWHA] wouldn't live long because the fact that you already got that stress on you. Medicine or no medicine I just couldn't function. I'd worry myself to death and you'll die sooner.

Shifting and changing priorities became evident when participants talked about their previous life experiences and how they viewed their incarceration experience through a different lens than did their younger incarcerated counterparts. Older AAPLWHA reflected on the circumstances that led to their incarceration and how it has impacted their life trajectory. Beau described:

When I look back on getting diagnosed in prison it changed my life. I mean I was older and it was a process for me getting to where I'm at today, not getting back to prison, not making it a part of my life anymore, actually accepting what happened. I don't know it helped change my life. It helped me get to...Not that my life is perfect today or anything, but it just helped me believe that something different was going to happen for me.

Like Beau, in hindsight many older adults came to view their incarceration as a catalyst for their improved HIV care. For many, it was the time when they either got diagnosed, became able to access care for the first time, or reengage in HIV care after a period of disengagement. Jethro explained "My incarceration I look at it as a blessing it is just something that saved my life. I am proud to be sixty years old with or without HIV. I'm still in good health." Similarly, Stew added, "I have been living with this [HIV] for a while now. I was diagnosed in prison and it changed a lot for me. I started taking the medication there and now I still take it and I just want to live and spend the time I still have healthy and free."

Experiencing ageism and structural racism was the third property that emerged from the analysis. Both properties were encountered by participants and impacted their incarceration experience. Participants described the experience of substandard medical 
care, as well as potential tensions with younger inmates. Not receiving adequate medical care while incarcerated was an occurrence many participants shared. Stew remembered, "I asked the doctor if there is any kind of therapy or treatment. He said no, all we can do is give you a wheelchair for three months and then you're on your own." Baby Bug described a similar experience:

They used to have a few doctors coming in to the prison but there were too many prisoners to take care of and just a few of them doing it. Then they did it over a TV monitor. It's still better than nothing but not real good care.

Several participants shared that the incarceration environment was not equipped to accommodate the needs of older AAPLWHA. Stew, for example elaborated on how the incarceration environment is designed for younger inmates "I am telling you climbing up those bunks and living in a cold cell with not enough heat is no place for older folks to live."

Additionally, some participants discussed tensions that emerged between younger and older inmates. Stew described, "the younger ones didn't like that we would sometimes have special privileges like a single cell or not being on the top bunk I think it would be better to be separated from the younger ones. I don't want to get in any fights no more at my age." Building on that, Midnight described the tension between younger and older inmates she observed during her time as a correctional officer. She said, "some of the younger inmates they didn't like the privileges some of the older prisoners would get like extra food and they let them know and they would discriminate and fight them." Overall, participants expressed that ageism and structural racism was present and impacted their incarceration experience.

In this chapter, I have presented the findings related to the first research aim of the study: How do older AAPLWHA draw meaning from their incarceration experiences? An exploration of the three sub aims addressed the influence of stigma (HIV and 
incarceration stigma), the different pathways through which the incarceration was experienced and how age and race impacted older AAPLWHAs incarceration experience. These understandings and meanings are influenced by behavioral and psychological processes older AAPLWHA engaged in.

Moving forward, the next chapter will address the second research aim: How incarceration experiences and understandings of their meaning relate to the process of engagement in care? The second research question's sub-aim will aid to understand the behavioral and psychological processes related to engagement in care for formerly incarcerated older AAPLWHA. 


\section{CHAPTER SEVEN: HOW DO INCARCERATION EXPERIENCES AND UNDERSTANDINGS OF THEIR MEANING RELATE TO ENGAGEMENT IN CARE?}

This chapter presents findings corresponding to the second research aim regarding how incarceration experiences and understandings of their meaning relate to the process of engagement in care. The sub-aim focuses on understanding the behavioral and psychological processes related to engagement in care for formerly incarcerated older AAPLWHA. The first section of this chapter will focus on behavioral processes and the second will focus on psychological processes.

\section{Understanding the Behavioral Processes}

\section{Behavioral Processes}
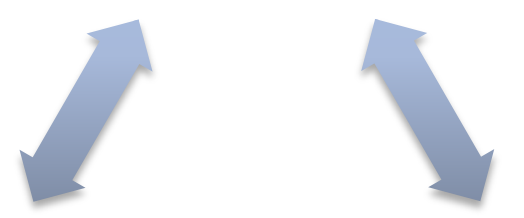

Engaging in care

Asserting self

Seeking out support (care)

Advocating own needs

"being taken care of"

Figure 7.1 Behavioral Processes
Disengagement in care

Keeping low

Isolating self

Avoiding care

Not disclosing status 
Figure 7.1 provides a visualization of the behavioral processes as they relate to engagement in care for older AAPLWHA. The behavioral processes were divided into two categories: engagement in care and disengagement in care. Engagement in care constituted of the following strategies: asserting self, seeking out support (care), advocating own needs, and being taken care of. Disengagement in care included keeping low, isolating self, avoiding care, and not disclosing status. This section uses supporting quotes to describe how each category (engagement in care and disengagement in care) was employed by older AAPLWHA in terms of the process of engagement in care.

\section{Engaging in Care}

Many participants found the time of their incarceration to be a catalyst for engaging in care despite facing challenges, such as timely access to care and privacy concerns. The challenges that participants experienced varied based on the respective correctional setting. These challenges, such as access to medication were often intensified when participants were incarcerated in a jail rather than a prison. Older AAPLWHAs engagement in care was characterized by asserting self, seeking out support (care), and advocating own needs. Participants often utilized more than one of the described strategies as they engaged in care. Each tactic required active involvement from the participants. Asserting self was one tactic participants employed as they engaged in care during the time of their incarceration. Billie asserted herself when it came to accessing her HIV medication. She shared, "I needed to tell them, let them know that I am a person and I need my medicine. They [meaning the correctional officers] didn't think I was important but I am a person just like them! I bug them and let them know that I don't stop until I have my meds." She, like others, asserted herself when it came to accessing her medication and benefitted from her persistance. Midnight, for example, shared "I showed them [correctional officers] I am confident and I'll get my 
meds. If you're timid you don't get nothing." There was a proactive element to this tactic in that it portrays confidence and determination. Asserting self was a behavior often combined with advocating own needs. Participants described how they actively advocated for their needs in terms of their HIV care. Rabbit shared, "I told them that I have to see the doctor. There was something not right with my medicine and I felt weak and was loosing a lot of weight. I told them [correctional officers] to let me see the nurse but they kept ignoring me. I kept talking to different guards and they got tired of me and let me go to medical." Similarly Zeus described, "I needed to eat with my HIV meds. I asked for extra food to take with my meds. The guard was nice about it went to the nurse and I got cleared for the extra snack. Later the nurse told me that I was the first one that has asked for that. I am glad I did though." The previous example illustrates how participants asserted their needs and consequently were able to positively impact access to medications and engage in care.

Another form of care engagement was actively seeking out support (care). This behavior was characterized by an effort by the participants to actively seek out available resources within their respective correctional setting. Such actions included seeking out the nurse, the participating in a support group (if available) for HIV positive individuals, and talking to a chaplain, a social worker or other mental health professionals about their circumstances. Unfortunately, such resources were scarce, and only a few participants described accessibility to supports. Jimmy, for example was incarcerated in a facility where a nurse at initiated a support group for HIV positive inmates. He described:

We were lucky there was a nurse in the facility and she started a HIV support group. I found out about it because I asked her if there's anything like that. They didn't make it a big deal or wrote it somewhere on the board it was more word of mouth. If I wouldn't have asked I wouldn't have known.

Similarly, Beau elaborated, "there was a support group for inmates with HIV. I went there it was helpful. I don't think many people went there because they was afraid 
because of confidentiality. They want know one to know that they are HIV." Midnight, like the majority of participants, was in a facility where no support group was offered for PLWHA. Seeking out support required initiative by her approaching the facilities chaplain. She elaborated, "I needed to talk to someone to get through this experience [being HIV positive and incarcerated] I kept asking around and the nurse told me that I can go and talk to the chaplain. That was helpful. She motivated me to stay engaged in care."

A final act of engagement in care included the acknowledgment of a service identified as "being taken care of." This engagement occurred when participants entered the correctional system and learned that they were eligible for medical benefits. "Being taken care of" represented free access to medication and treatment in a contained environment that often facilitated adherence to treatment and medication regiment. Zeus experienced this, "I had a sense of being taken care of. I mean in the facility I didn't have to pay for medical care it was all free and they would give them medicine to me everyday I never missed." Similarly, Beau who was diagnosed while incarcerated described, "If I would have found out [meaning HIV positive status] on the outside I couldn't afford the medicine and all that you know. I probably would not have cared and died from this." Jimmy also described the process of being taken care of by a medical provider "the nurse that we had we could always go to her and talk to her about anything that was going on if we had any symptoms or problems with our medicine. To me she was like a rock." While all participants were "being taken care of" in terms of their eligibility for medical benefits, this facilitated engagement in HIV care for some more than for others.

\section{Disengagement in Care}

The second behavioral process impacting care related to the rejection of care in one form or another. Disengagement in care included keeping low, isolating self, 
avoiding care, and not disclosing status. Keeping low was a tactic widely used by participants, and resulted in disregarding care. Doc's experience illustrates this. He described:

I had to keep low because no one can know that you are HIV. The deal is other inmates want to know what's going on they're asking questions if they see you take meds or go to the nurse a lot. They'll ask why did you go to the nurse and what's that medicine for? And if they find out that's it they'll beat you to death. It's a brutal world in there. That's why I skipped my meds.

Other participants were keeping low by taking their medicine back to their cell and hiding it so they could take it in secret when their cellmates were not present or asleep. Billie spoke to this experience. She explained, "I would hide my meds from my cell mate and wait until they are asleep then I'll take it. I wasn't supposed to do it that way. I need to have it in the morning with food but that wasn't an option."

Isolating self was a second tactic that kept others at a distance and resulted in disengagement in care. Participants explained how they would keep to themselves and avoid contact with other inmates, fearing that engaging would increase the risk of negative consequences, such as being outed as HIV positive, discriminated against, or attacked. Zeus explained, “I kept to myself I don't want no one in my business. Most of the time I was by myself I didn't want someone to get to know me and find out about my status. It was better to stay safe." While participants explained that isolating themselves was a protective measure, many acknowledged the negative impact of isolating in regards to HIV care. Jimmy explained, "I kept to myself mostly but I got depressed and then I didn't care if I was taking my meds or not. There was no point to all of this you know."

A third strategy by which to disengage care was through intentionally avoiding care. Many older AAPLWHA avoided care by either not paying attention to their HIV related symptoms and/or skipping HIV medications. Participants feared consequences such as being discriminated against, being treated differently, labeled or judged, which 
led to them to avoid care. James mentioned:

I know a guy that didn't take his medication he turned it all down in prison. They [correctional officers] said oh you ain't got to take your medicine and you ain't got to see no doctor. If you don't want treatment, we can't force you to take it." I don't know what happened to him when he got out but inside he didn't take any medicine.

Finally, the fourth strategy participants used to disengage in care was to "not disclosing their HIV status." This occurred behavior occurred when participants, who were aware of their HIV status before incarceration, decided to not disclose their status for fear of experiencing stigma and discrimination. Midnight for example decided to not her status due to her experience as a correctional officer and having seen HIV positive inmates facing stigma and discrimination. She stated, "no I decided to tell nobody about my HIV when I was in jail I know what goes on in there from my time as a correctional officer and I didn't need that." The underlying fear behind "not disclosing HIV status" was related to stigma and discrimination. The cost was not receiving crucial medical care. This choice was more prevalent in participants who were incarcerated in a jail compared to a prison.

\section{Understanding the Psychological Processes}

As illustrated in Figure 7.2 the psychological processes related to engagement in care for older AAPLWHA during their incarceration can be viewed from a temporal perspective focused on past, present, and anticipation - future. 


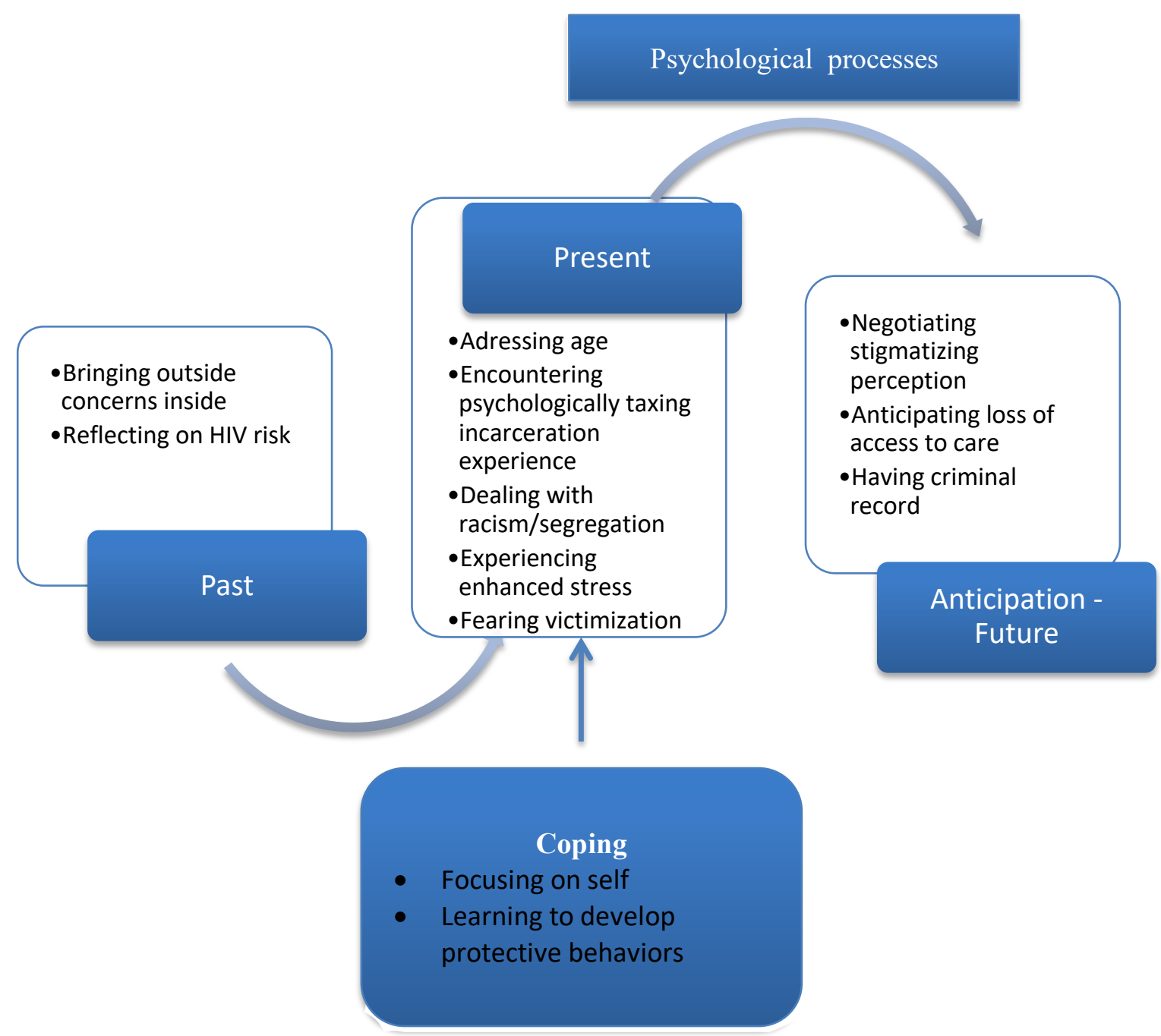

Future Coping Strategies

- Seeking out social and/or peer support

- Securing housing

- Religious coping

- Searching for meaning

Figure 7.2 Psychological Processes related to Engagement in Care for formerly incarcerated older AAPLWHA 
Psychological processes relating to the past were described by "bringing outside concerns inside" and "reflecting on HIV risk." The first behavior, "bringing outside concerns inside" differed for older AAPLWHA who were diagnosed with HIV prior to their incarceration. Participants expressed concern that they would not receive the care they needed to sustain their health once incarcerated. Jimmy described his experience of needing a colonoscopy while incarcerated but was unable to do so. Jimmy explained:

I was concerned about, I am older I needed a colonoscopy, so I asked the nurse. She said, oh, we don't do preventative measures here. You just have to wait until you get out. I was like, are you serious? You know I'm HIV positive and you know, colon cancer runs in my family. You ask me every time, has anybody had colon cancer in your family, and I say yes. So you mean to tell me you're not going to check me? I got to wait until I get out. I said, okay, if I get out and they say I got cancer, I'm coming back. Not to get in prison, but my lawyer be back. They wouldn't do it.

Others worried about their privacy and feared that their HIV diagnoses would be disclosed to others. Billie recalled her fears when her HIV status was disclosed:

Regarding confidentiality the guards, they all knew. Every guard knew about my status. Some did keep it confidential and some didn't. Like some inmates would come to me and say, "We know you have HIV," and that the guards had told them. They said, We don't know what so-and-so [meaning the guard] got against you, but he came back and told us that you had HIV. Then I knew I was outed.

A second process that was described by older AAPLWHA involved reflecting on past HIV risk. Older AAPLWHA often searched back in their life experiences to determine at what point they might have been infected with the virus. Lil Booger's reflection on his HIV risk illustrates this process, "You know while I was incarcerated I had a lot of time to sit and think. I reflected on my life and this disease. How did I get it? I know I wasn't protecting myself I see that now but back then when I was younger that wasn't on my mind." 


\section{Present}

The second psychological process older AAPLWHA engaged in was the present. Older AAPLWHAs descriptions of their incarceration experience included narratives about addressing age, encountering psychologically taxing incarceration experiences, dealing with racism/segregation, experiencing enhanced stress, and fearing victimization.

\section{Addressing Age}

Participants agreed that incarceration settings were ill equipped to meet the needs of an aging population, let alone older adults living with HIV. Addressing age was a pressing concern for older AAPLWHA as they often spoke of what it meant for them to be older and incarcerated. Zeus spoke to that reality:

They don't understand that we are older and we have different health issues than the younger ones. The bunks we can't climb on them anymore and then we've got HIV on top of that. It took a strain on our bodies. I might not be 70 yet but my body sure is.

Similar to Zeus, Jethro described his experience of dealing with age while incarcerated. He shared, "If you are older and incarcerated it is much harder on your body and your mind you feel like you are going to die in here."

\section{Encountering Psychologically Taxing Incarceration Experience}

The incarceration experience for older AAPLWHA was psychologically taxing. Participants described the experience of confinement and the loss of freedom coupled with interpersonal distrust, which often led to a diminished sense of self-worth and personal value. Stew spoke to this:

You know now I was locked up I couldn't go where I wanted or make my own decisions every day. I felt trapped in my mind I still had to deal with my HIV and everything but it was all out of my control I just had lost everything in my life.

Similarly, Midnight shared, "I didn't feel like a person anymore all that I had was taken from me that's what I felt like." 


\section{Dealing with Racism/Segregation}

Older AAPLWHA recounted stark disparities within correctional facilities in terms of racism and segregation. Older AAPLWHA dealt with structural racism where segregation (due to HIV status and/or race) was prevalent. Racism was evident in the form of institutional structural decisions that negatively impacted older AAPLWHAs. Participants described that due to their race they often felt like they received the brunt of racism, meaning longer sentences and fewer privileges than their other racial counterparts. Employment opportunities and access to programs were noted as examples. Zeus voiced his concerns, "they sending Blacks to prison for longer and once we are in there they don't give us the same privileges that white folks get."

While some participants preferred to be segregated from the general population, others felt singled out and feared that their HIV status was made obvious to other inmates. Doc shared how living in a segregated dorm made him feel, "They [other inmates] knew we had HIV because they had us in the HIV dorm." Stew, on the other hand would have preferred to be segregated from the general population. He shared, "I would have felt saver if we [meaning other HIV positive inmates] would have been in a different dorm." As Stew described, the nature of the prison environment manifested in a desire among some participants for segregation within the correctional facility in order to protect themselves from harassment.

\section{Experiencing Enhanced Stress}

Another psychological impact of experiencing the present was the increase in stress levels. Participants shared the realities of restricted movement, a nearly total lack of personal privacy, high levels of interpersonal uncertainty, and a fear of being exposed as having HIV. The constant focus on these uncontrolled circumstances adversely impacted older AAPLWHAs emotional well-being. Billie described, "I was scared that 
someone finds out my status. I lived in fear all the time it was very stressful. It gave me depression." Stew agreed, "I couldn't go where I wanted to go I was locked and I had to do what I was told it was a stressful time for me." A correlation between enhanced stress and the fear of being victimized was apparaent.

\section{Fearing Victimization}

Fearing victimization during their period of incarceration was a common psychological stressor for older AAPLWHA. Such victimization experiences included psychological, physical and sexual forms of violence. Participants described that their HIV status made them more vulnerable for victimization. Male participants overwhelmingly described that they were assumed to be gay, and therefore were at heightened risk for sexual assault. Jimmy shared his experience of fearing victimization:

One time, when I was in the jail I was sleeping, I woke up one morning, and there was toothpaste on my blanket. I'm like, okay, who did this? I had an idea who did it. Later on that day, the guy came to me. He said, I saw you out there talking. I said, yeah you the one that put the toothpaste on my blanket and things while I was sleeping. I said, you're a coward. If you gay have HIV or whatever, they would do things to you. That's the only thing that really happened to me. I'm blessed that I never got hurt or got beat up, that's the only little thing that happened.

Lil Booger described:

Other inmates, they're throwing shit in my cell, pissing in my cell, spitting in my food. The guards were spitting in my food. Listen, they would even... I'd be in the back of my cell. I would wash up by the sink. I wouldn't come out to take a shower, because for fear of my life. I had to file a court order so I can take a shower. See, all they [guards] had to do was take me because they was doing, basically, they take a guy out, go down to the end of the hall, and they'd escort him through the shower. Let him shower and come out.

\section{Anticipation - Future}

The third psychological process was linked to anticipation - future and included negotiating stigmatizing perceptions, anticipating the loss of access to care, dealing with the consequences of having a criminal record and taking charge of one's health. One of the most prominent issues for participants post-release was confronting the stigma 
associated with their incarceration experiences. Such behaviors included negotiating stigmatizing perceptions by the general public, potential employers, health care providers, and their community and families. Beau spoke to that, "Once you get released the stigma is bad. There are rumors about you. You're older and you've been incarcerated and in my case my family they know about the HIV it is a lot to deal with." Likewise, Billie discussed the stigmatizing perceptions she encountered from others, "they [others] make assumptions about you because you have been incarcerated. I went to the doctor after I was released and the nurse she gave me that look like I was dirty or something because I was in the penitentiary and have HIV." Upon release, participants anticipated the loss of access to health care, which led to elevated levels of anxiety and psychological stress. Eminem described, “When I got released I didn't have no insurance. They gave me three days of pills when I left and then I was on my own." Participants described a lack of knowledge about how to obtain care once they were released. Doc said, "Inside they get you everything your meds and stuff every day you get in line and they hand them to you then you get out and nothing." Loosing access to care was most prominent for participants who were incarcerated in a prison.

\section{Dealing with the Consequences of Having a Criminal Record}

The consequences of having a criminal record were far reaching for participants. Such consequences included identified and unidentified barriers. Identified systemic barriers included the inability to vote, hardships when securing employment, or being barred from subsidized housing and public benefits. Unidentified barriers could include experiencing prejudice and discrimination within the community and maintaining or reframing relationships with friends and family members. Eminem shared, "They won't let you vote if you have a record and it is hard to get a job. I tried before and they wouldn't hire me because I was a felon. I try not to let it get to me." Similarly, Beau 
described, "I felt the stigma that you get when you are a felon. My community, family is the one thing and then it is also tough to find a place to live."

\section{Taking Charge of One's Health}

Individuals returning to the community following release faced a challenging transition period which included taking full responsibility for managing HIV. While incarcerated, decisions about health care were often made for the participants. Even those who chose not to disclose their HIV positive status had health issues related to age monitored by institutional health providers. Once released, participants where, for the most part, completely on their own in regards to maintaining their health. Most participants described great difficulty in accessing HIV treatment and health care. Billie spoke to this, "Once you get released they give you a weeks worth of your HIV meds and then you are on your own. I had so much to deal with when I got out and that was on top of all of it."

Jimmy was one of the few participants who described a successful linkage to care post-release, "I didn't go to the 550 clinic until I went to prison and they did in the prison connect me to them. I just kept with them being with them since I got out." For some participants the adjustment post-release was difficult to maintain any healthy habits that had been developed while incarcerated. Red described,

When I was incarcerated I was exercising and stuff, but when I left that I stopped doing it. I stopped drinking Ensure and I stopped doing all other thing. I started doing things to my body like smoking and drinking and using drugs as if the HIV had never came into my body or something.

Participants revealed two coping strategy phases within the temporal psychological process. The first coping strategy was linked to present coping, namely focusing on self and learning to develop protective behaviors. Billie spoke to this "when I was in there I was trying to take care of just myself and not focus on what's going on around me. I learned to protect myself." Eminem described the protective behaviors that 
he had developed in detail, "I didn't take my meds until my roommate was asleep. I also made sure that no one knew what kind of doctor I am seeing." The second coping strategy linked to anticipating the future, whereby, participants predicted what kind of tactics they would engage in post-incarceration. These tactics were developed by reflecting on past psychological practices and creating new ones. Tactics included seeking out social and/or peer support, securing housing, religious coping, and searching for meaning. Beau spoke to this, "I was trying to make some change when I got out that time. I looked for a support group and made sure not to stay to myself. I wanted my life to change and go in a positive direction you know."

This chapter presented findings corresponding to the second research aim regarding how incarceration experiences and understandings of their meaning related to behavioral and psychological processes of engagement in care. 


\section{CHAPTER EIGHT: AN INDUCTIVE THEORY OF THE PROCESS RELATED TO THE INCARCERATION EXPERIENCES AMONG OLDER AAPLWHA AND THEIR ENGAGEMENT IN CARE}

\section{Overview of the Chapter}

This chapter begins with an overview and introduction to the inductive theory “Older AAPLWHAs journey towards engagement in care during incarceration." As a researcher, my goal was to understand the process related to the incarceration experiences. Of particular interest was accurately describing participants' lived experiences of being HIV positive, older and AA while incarcerated. Listening to participants' voices helped me to understand older AAPLWHAs efforts to engage in HIV care, their struggles and identified needs. Therefore, the inductive theory "Older AAPLWHAs journey towards engagement in care during incarceration reflects a core category "It's hard being locked up, old, Black and HIV. We're not seen" that is representative of the inductive theory. The inductive theory explains how participants engaged in the process of HIV care from the point of entry to post-release. The theory is represented by four categories (phases) and sixteen meaningful concepts that are grounded in the data. This chapter also describes the internal and external factors (context) affecting participants' engagement in HIV care, and the process related to older AAPLWHAs incarceration experiences. These factors were noted as facilitating or limiting conditions that advanced or delayed participants' ability to engage in HIV care while incarcerated. 


\section{Overview of the Inductive Theory "Older AAPLWHAs Journey towards Engagement in Care during Incarceration"}

The second aim of this constructivist grounded theory study was to develop an inductive theory explaining the process related to the incarceration experiences among older AAPLWHA as well as their engagement in care. The inductive theory "Older AAPLWHAs Journey towards Engagement in Care during Incarceration" explains how older AAPLWHA engaged in the process of HIV care during incarceration. The inductive theory "Older AAPLWHAs Journey towards Engagement in Care during Incarceration" is also a social process reflected through four phases. These phases are linked in a process that occurs over time and changes over time. The four phases also represents the transition of participants moving through the incarceration experience to post-release in regards to HIV care engagement. The components of the inductive theory are comprised of the following four phases: 1) Entering the Correctional System; 2) Developing Understandings of HIV within the correctional system; 3) Engagement in HIV care; and 4) Linkage to care post-release. The transition through the phases was gradual, or occurred multiple times in the case of more than one incarceration. Each phase represented a process within itself and was able to stand by itself. For some participants "developing understandings of HIV" led to a gradual engagement in care. Therefore, the theory "Older AAPLWHAs Journey towards Engagement in Care during Incarceration" entailed a progressive movement and consisted of four phases. Each phase is represented by a category and its respective set of meaningful concepts. The concepts allowed for the following: 1) framing key characteristics of individuals' experiences, 2) communicating experiences in the language of the participants, and 3) constructing relationships among the concepts. The theory with its phases, categories, context and participants voices are depicted below in Figure 8.1. 


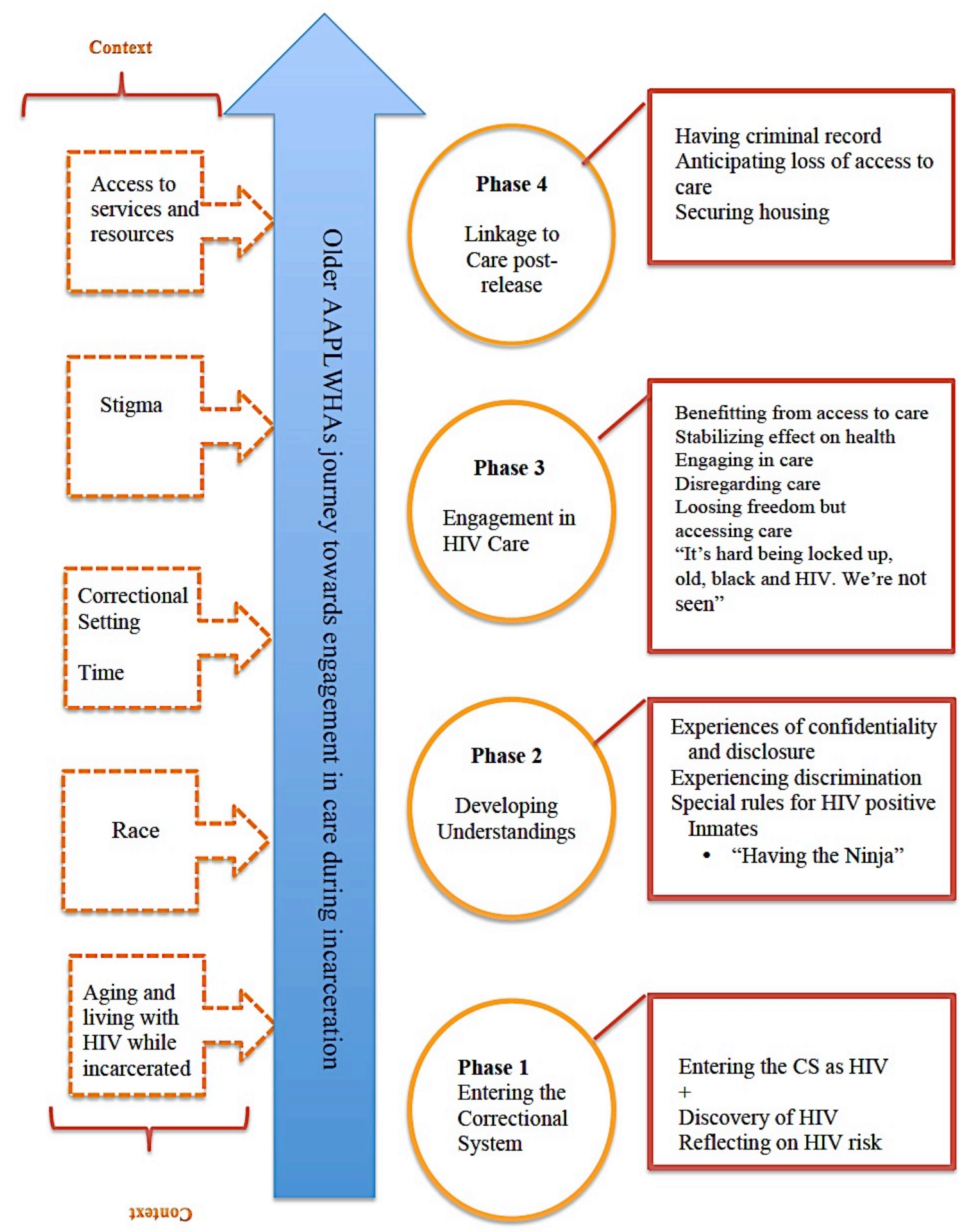

Figure 8.1 Model Representation of the Inductive Theory depicting the Phases and the Context 
The four phases of engagement follow the trajectory of each participant's experience from entry to release. The first phase constituted "Entering the Correctional System" in which participants either were already diagnosed with HIV or discovered their HIV positive status during their incarceration. Participants reported the discovery of their HIV status while incarcerated as a life-altering experience. For example, James described, "when I went in [prison] that's when I got tested and found out I must have had it [HIV] for at least 14 years by then. That was a shock." Discovering their HIV status triggered thoughts as to how they had contracted the disease as well. Zeus, who was diagnosed while incarcerated stated, "I started thinking back on my life what I did with whom I was and how I've gotten it [meaning HIV]."

As previously described, the process of entering the correctional system differed depending on the pathway through which the incarceration was experienced, such as prison versus jail. Participants whose incarceration experience took place in a jail often expressed that HIV testing was non-existing and resources for HIV care engagement were sparse. In comparison, participants who were incarcerated in a prison setting often had access to HIV testing and more resources that allowed them to engage in HIV care.

The second phase consisted of "Developing Understandings of HIV within the correctional system" and understanding what it means to live with HIV while incarcerated. This phase included experiences of confidentiality and disclosure, experiencing discrimination, as well as identifying special rules for HIV positive inmates. One particular meaningful concept was "having the ninja" that was used as a slang reference to an incarcerated individual having the HIV virus.

The third phase constituted Engagement in HIV care. As participants approached the third phase understandings of what it means to live with HIV within the correctional setting had been developed; those understandings were instrumental for setting the stage 
for either HIV care engagement or disengagement. As previously stated, HIV care in the incarceration environment was overshadowed by institutional barriers such as access to medical providers. This was especially difficult for participants who were incarcerated in a jail. In many instances, care was provided via telehealth where participants were not able to see a physician face to face. Receiving substandard medical care during incarceration was also a result of the fact that the incarceration environment was designed for younger individuals. The older age and the HIV status of participants introduced additional challenges for functional ability, safety and health, such as an increased vulnerability to infections such as pneumonia. Experiencing stigma, fearing discrimination, and lacking access to mental health services were common barriers that impacted HIV care engagement for participants. Lacking access to mental health care was also a prominent barrier for participants. While not all participants experienced serious mental health problems, many described experiences of depression, stress and psychological trauma due to incarceration.

Phase four constituted Linkage to Care post-release and included participants' experiences as they left the correctional system and began their transition back into their communities. This phase was overshadowed by great barriers, such as the lack of access to care, challenges in securing housing, and a lack of personal agency to coordinate their own HIV care or locate ASOs in their communities. Upon release, participants often found themselves returning to living in communities that lacked access to public transportation. Further, participants were often not able to navigate the steps to obtaining the crucial and live saving medical insurance.

The four phases of the inductive theory "Older AAPLWHAs Journey towards Engagement in Care during Incarceration" also accounted for the internal and external factors that affected participants' engagement in HIV care. These factors were accessing 
services, obtaining resources, addressing stigma (HIV stigma and incarceration stigma), the correctional setting, race, aging and living with HIV while incarcerated and the time of the incarceration which ranged from the early $1980 \mathrm{~s}-2015$.

The following section will provide two case exemplars, which were chosen to demonstrate the process represented by the inductive theory, "Older AAPLWHAs Journey towards Engagement in Care during Incarceration." While all participant narratives offered insight into the process, these two case exemplars differed significantly in their experience when it came to engagement in care during incarceration. Billie and Beau, the two participants, represented the youngest age group $(50-60$ years $)$ but differed in other characteristics. Billie identified as transgender, was unsure regarding her sexual orientation, and was not diagnosed with HIV while incarcerated. Conversely, Beau was male, identified as heterosexual, and was diagnosed with HIV while incarcerated.

\section{Billie}

Billie had been incarcerated multiple times. Three of her incarcerations took place in a state prison. However, she was unsure how many times she was incarcerated in a jail, stating, "I can't tell you how many times I was locked up in jail for this or that." Billie received her HIV diagnosis outside of the correctional setting, and was aware of her HIV status at each incarceration. Despite entering the correctional setting and being aware of her HIV positive status, she described each incarceration as a time when she looked back at her life and reflected on her HIV risk. She said, "each time I was locked up I looked back at my life and thought maybe that was the time I got infected." Since Billie repeated phase 1 "Entering the Correctional System" multiple times she reported increased anxiety with each incarceration. She stated, "the more you get locked up you know what to expect when you go in and you don't get the care you need." Further, with 
each incarceration Billie transitioned more quickly between phase 1 "Entering the Correctional System" and phase 2 "Developing Understandings." She explained, "the second time I went in [meaning prison] I kind of knew what the deal was when you have it [HIV] and you just go from there." Billie's understandings of what it meant to live with HIV within the correctional setting did not dramatically change with each incarceration. However, she developed a clearer picture of the challenges that accompany incarceration with an HIV diagnosis. She explained, "You know what to expect but I feared for my life more and more each time you learn different things the more you're in [correctional setting]." For Billie, developing understandings of what it means to live with HIV while incarcerated included the following: the fear of and the experience of having her confidentiality broken, experiencing disclosure violations about her HIV status, and being subject to special rules for HIV positive inmates. During her interview Billie elaborated on how the nurses broke confidentiality. She said,

They [meaning nurses] will spread your business in a heartbeat. Yes. Sorry to say it. I'm sorry to put that out there, but it is a true fact. I've seen it with my own two eyes. I have had nurses in the prison system that have been cool with me, and comfortable enough to come and show me the list of names of people that they knew [meaning HIV positive] was on there and others told me that the nurses did the same with my name to others.

Moreover, Billie spoke to the discrimination within the correctional system. She elaborated,

Comparing to a Caucasian person with HIV you [being AA] already not allowed to join in a lot of the gangs and groups and stuff. That's the way they treat each other in prison. Being Black and happen to deal with HIV in that aspect there is a lot going on with Black on Black abuse. I mean physical, mental ... Joking turns into rage, rage turns into assault, and it trickles down. It goes from penitentiary to penitentiary. They try to move someone to somewhere else or to another facility, but they treat you in the heartless way when they find out that you are HIV. That's true for guards and other inmates. 
Billie's understanding of what it means to live with HIV in the correctional setting was also shaped by observing and experiencing "special rules for HIV positive inmates." She said,

When they [correctional officers] find out they change way they treat the person with HIV. I felt it... they have usual cell checks, and in those cell checks on a person who is not HIV the officer would come in with no gloves. When it comes to a cell check with a person that they know is HIV positive or are gay, they have gloves on. In one way I think it is for protection, but then in other ways I think it is shaming because you're showing difference in the inmates. You're supposed to treat each inmates the same. You're showing that "Okay something's wrong with this inmate," and the other inmates are looking at that and are like Okay, why are you wearing gloves on this one but you was just down the hall and you didn't have gloves on?

The context in which Billie experienced the four phases depended significantly on her various incarceration placements. Although she moved more quickly between phases 1 and 2 because of her familiarity with the incarceration process, phase 2 "Developing Understandings," was overshadowed by stigma, differential treatment based on race, and discrimination that took many forms. Those negative experiences contributed to her transition into phase 3, "Engagement in HIV care." As Billie's understandings of what it means to live with HIV while incarcerated expanded and changed, she decided to disengage in care during incarceration. For Billie, despite being able to access care, the stigma and stress she experienced left her feeling as if the only way to cope and feel physically and psychologically safe was to disengage in HIV care. She elaborated,

Being Black with HIV and transgender is a hard life. In the Black community to be transgender is one problem but being a transgender in prison they already have a conclusion that they want to hurt you. I did not see the doctor or took my meds the right way. I know that wasn't good for me but I just had to deal.

The fourth phase, Linkage to Care post-release, was also a challenge for Billie. Immediately after release Billie was not provided with resources connecting her to a local HIV clinic or ASOs. She described that following each release she did not engage in care for an extended period of time. She said, "I had to deal with the trauma of being locked 
up and all my fears I just could not do one more thing [meaning seeking care] I had to find housing and work you know I had to live somehow and with getting older each time things were tough."

Overall, Billie's journey towards engagement in care during incarceration was one overshadowed by stigma, discrimination, inconsistent correctional environments, and challenges navigating the stressors of aging with HIV.

\section{Beau}

Like Billie, Beau was also incarcerated multiple times. Unlike Billie, he was diagnosed while incarcerated in a prison. Upon entering the correctional system he requested to be tested for HIV (Phase 1 "Entering the Correctional Setting"). At the time of his incarceration, he read an article about HIV at the doctor's office at the state prison, which made him reflect on his own HIV risk, "I asked them to test my blood because I saw things on TV and read this magazine then they tested me and later they called me back to the doctor's office and told me I was positive." Beau's experiences with maintaining confidentiality and disclosure were mostly positive. However, he described one instance where he felt stigma from other inmates that were not HIV positive. He states, "I felt a little stigma sometimes not towards myself it was just the other prisoners they judge HIV positive inmates." For Beau, the experience of entering Phase 3, "Engagement in HIV care" for Beau was significantly different than for Billie. He describes the lifesaving experience of being diagnosed while incarcerated and being able to access care. Beau remembered,

Actually, I'm going to tell you something. When I look back on getting the diagnosis in prison. It saved my life. It was a process for me getting to where I'm at today I am not going back to prison, but being able to be diagnosed when I was there changed everything. I got them medicines and care and I actually accepted what I had [meaning HIV], even though I wasn't always listening to the healthcare workers and everything. I don't know; it helped safe my life. Not that my life is perfect today or anything, but it just helped me believe that something different was going to happen for me. 
For Beau entering Phase 4 "Linkage to Care post-release" succeeded because of his earlier positive transitions through phases $1-3$. Following his incarceration, Beau was successfully linked to care and was able to stay engaged in care.

In this chapter, I presented an inductive theory of the process related to the incarceration experiences among older AAPLWHA, and the four phases that defined their engagement in care. The process depicted by inductive theory was then applied to two case exemplars, which differed in their experience of engagement in care during their incarceration. Even though participants' experiences differed, the processes, categories and concepts of the inductive theory were applicable to a variety of older AAPLWHAs experiences, which demonstrated the abstract nature of CGT research. 


\section{CHAPTER NINE: DISCUSSION}

The purpose of this constructivist grounded theory study was to understand the processes older AA adults experience related to their incarceration and engagement in care. The previous chapter presented an inductive theory of the process related to incarceration experiences among older AAPLWHA and their engagement in care. This chapter begins with the conclusions from this study, following the study's two primary goals 1) to understand and provide a deep description of the experiences of incarceration among older AAPLWHA, and 2) to develop an inductive theory of the process related to incarceration experiences among older AAPLWHA and their engagement in care. The conclusions therefore, address the major findings related to the two aims of the study: 1) How do older AAPLWHA draw meaning from their incarceration experiences, and 2) How do incarceration experiences and understandings of their meaning relate to the process of engagement in care and a discussion of findings in relation to the existing literature. The subsequent section focuses on further discussion of research findings, limitations of this study, and suggestions for future research. This is followed by the researcher's recommendations, and a final reflection.

\section{How do Older AAPLWHA Draw Meaning from Their Incarceration Experiences?}

Regarding the influence of stigma, participants experienced stigma from their incarceration status and diagnosis with HIV. While HIV stigma was prevalent during the incarceration experience, incarceration stigma was most apparent for participants' postrelease. The finding that incarceration stigma overwrote HIV stigma is unique to this study, and has not been embedded in the existing literature. In one study, Brinkley- 
Rubenstein (2015), recounted the experiences of formerly incarcerated AA men and the multiple stigmas they encountered. The study found that participants described equally high levels of HIV and incarceration stigma and did not experience one form of stigma more than the other. It is feasible, that for the participants of this study, HIV stigma was to some extent more controllable than incarceration stigma. This was especially prevalent post-release. For example, in certain situations such as job interviews, individuals are not required to disclose their HIV status. Incarceration stigma, on the other hand, was unavoidable when applying for a job and participants subsequently had to release the information that they had an incarceration history. Therefore, it is understandable why participants would experience higher rates of incarceration stigma, since they had more control over protecting their HIV status related stigma. Their HIV-positive identities were often more concealable than their incarceration history.

Interestingly, two participants reported that they did not experience stigma during their incarceration experience while the majority of participants described experiences of internal and/or external stigma on multiple levels (Figure 6.1), as well multiple stigmatizing identities (Figure 6.2) in terms of incarceration stigma. It is possible that the following circumstances contributed to these two participants not experiencing stigma: The offer of a support group for HIV positive inmates; a positive experience of segregated housing while incarcerated; multiple incarcerations; a supportive family environment, and being long-term survivors of HIV. Long-term survivorship potentially contributed to decreased experiences of stigma. Long-term survivors lived through decades of extensive levels of stigma and might have developed positive coping mechanisms (Emlet, Harris, Furlotte, Brennan \& Pierpaoli, 2017). Moreover, those two participants reported increased HIV care engagement while incarcerated, and were able to 
access services with more ease. In retrospect, both participants considered their incarceration as lifesaving and transformational in terms of their HIV care engagement.

In contrast, the majority of participants described experiences of internal and/or external stigma on multiple levels, as well multiple stigmatizing identities in terms of HIV and incarceration stigma. These findings align the challenges noted in existing literature regarding PLWHA in incarceration settings (Brinkley-Rubinstein, 2015; Zawitz et al., 2014; Andrinopolous et al., 2010; Juergens et al., 2011).

Another interesting finding was that the experience of HIV stigma was perpetuated by the nickname "the ninja" by which incarcerated individuals referred to the HIV virus. Referring to HIV as "the ninja" can be viewed in the context of symbolic interactionism. Individuals use symbols to contribute to meaning making experiences and inform actions (Charmaz, 2014; Glaser \& Strauss, 1967; Strauss \& Corbin 1998). In the context of this study, shared symbols such as "the ninja" were used to communicate meaning of what it means to live with HIV in the incarceration environment. The personification of HIV as a black clothed figure that sneaks up on a person and attacks, contributed to the meaning making process for participants in the sense that the HIV virus was something that can happen at any time, is painful, comes unexpected, and should be feared. Therefore, individuals who have "the ninja" taken on the manifestation of the symbol and its meaning.

The symbolic nature of living with a stigmatizing disease in the incarceration environment resulted in distancing themselves from their HIV status, often through not disclosing their status, disengaging in care and fear of discrimination. Moreover, this association contributed to the understanding that there is a pervasive stigma among inmates, which overshadowed participants' incarceration experiences. Additionally, this finding relates to the theoretical concept of the meaning making process, which was 
developed by Park and Folkman (1997) within the broader framework of Lazarus' stress and coping theory (Folkman \& Lazarus, 1984; Lazarus 1993). Park and Folkman (1997) described that only through meaning making are individuals able to cope with stressful life circumstances, such as a stigmatizing disease. Plattner and Meiring (2006) applied Park \& Folkman's (1997) theoretical concept of meaning making to explore how PLWHA cope with the circumstances surrounding living with HIV. Their study revealed the importance of acceptance of the HIV diagnosis, and included narratives of deservingness and self- blame when it came to meaning making discourses and coping. For many participants of this study, carrying "the ninja" inside oneself equated to feelings of fear, self-blame, and narratives of deservedness and social stigmatization. Overt and subtle stigmatizing messages were perpetuated by a lack of education in regards to HIV. Participants explained that correctional officers, the administration, and other incarcerated individuals lacked crucial education when it came to HIV and its transmission. This finding is consistent with existing literature that highlights a lack of education among correctional officers and incarcerated individuals (Alarid \& Marquart, 2009; Juergens et al., 2011).

An exploration of the pathways through which participants experienced their incarceration resulted in the identification of jails and prison as the two major pathways (Figure 6.3). Incarceration in jail was characterized through experiencing an ever changing environment; whereas being in prison was characterized through experiencing permanency. While in jail, participants were often denied lifesaving medications and stalled when asked to access their HIV medication. Overall, more barriers to access to care were experienced in jails than in prisons. Upon entering the prison environment, participants were often diagnosed and/or connected to HIV care for the first time. Studies have shown that incarceration is a chance to test for HIV and connect to care (Ammon, 
Iroh, Tiruneh, Montague et al., 2018; Harawa et al., 2009; Iroh et al., 2015; Meyer, Cepeda, Taxman \& Altice, 2015; Westergaard, Spaulding \& Flanigan 2013). The results of this study align with previous research stating that prisons play a vital role when it comes to testing and engagement in HIV care (Iroh et al., 2015; Maruschak et al., 2009; Rich et al., 2013). Prisons are often the first step in the HIV care continuums sequential steps from initial diagnosis to the goal of viral suppression. It is important to note that the participants of this study were incarcerated at different points in time and some in states outside of Kentucky. Further, the state of Kentucky does not routinely test inmates for HIV in correctional settings and mandates only a basic health screening (Kentucky Department of Corrections, 2017). One of the improvements that needs to be made is to implement the CDC's recommendation of implementing at least opt-out testing (Beckwith et al., 2012, CDC, 2015). This policy change reflects a first step to eventually testing all inmates for HIV upon admission and before release.

In terms of how age and race impacted participants' incarceration experience three properties were identified: accelerated aging, "dying anyday", and ageism and structural racism. Consistent with existing literature, participants described accelerated or accentuated aging, and the experience of chronic illness and disability (Falter, 2006; Martin \& Volberding 2010; Pathai et al., 2013; Trotter et al., 2015; Yarnell, Kirwin \& Zonana, 2017; Williams, Stern, Mellow, Safer et al., 2012). Further, some participants described experiences of what they interpreted as their body aging faster during incarceration. This finding is echoed in previous research describing that incarcerated individuals are physiologically about 10 years older than their chronological age (Aday \& Krabill, 2012; Falter, 2006; Noworny et al., 2016). Additionally, correctional facilities were ill equipped to meet the needs of an aging incarcerated population, which has been documented in the literature (Aday et al., 2012). 
Interestingly, in the context of aging the participants of this study described the experience of shifting and changing priorities. Older AAPLWHA reflected on the circumstances that led to their HIV diagnosis and/or their incarceration and the impact it had on their life trajectory. Thereby incarceration (in a prison) was often identified as the major catalyst to engagement or reengagement in care. However, substandard medical care not only for HIV but also for other age-related conditions and potential tensions with younger inmates, reflected the notion that the incarceration environment is designed to meet the needs of younger inmates. These findings facilitate and add to the understandings how older AAPLWHA draw meaning from their incarceration experiences.

The findings of this study revealed that participants carried multiple stigmatizing identities which can be discussed in the context of intersectionality. Intersectionality is a theoretical framework that posits that multiple social categories (e.g. age, race, gender, sexual orientation) intersect and create overlapping and interdependent systems of discrimination (Collins \& Bilge, 2000). Participants carried multiple stigmatized and marginalized identities in terms of their HIV status, age, race, and incarceration history. These overlapping identities led participants to experience cumulative disadvantage and societal discrimination. Even though this study was conceptualized using the CHSCP, SI, and SI stigma as theoretical sensitizing concepts, the framework of intersectionality related to older AAPLWHAs experiences is potentially more theoretically explanatory in terms of how participants experienced prejudice based on which identity they concealed and which identity was presented. Certain marginalized identities, such as being Black were never able to be concealed. Combined with incarceration stigma, this lead to participants facing discrimination, such as losing employment opportunities post-release. 


\section{How do Incarceration Experiences and Understandings of Their Meaning Relate to the Process of Engagement in Care?}

Behavioral and psychological processes influenced the meaning making process of the incarceration experience for older AAPLWHA. The behavioral processes were characterized through two categories. The first category was engagement in care and the second category was disengagement in care. Participants employed each category in terms of the process of care engagement (Figure 7.1). Older AAPLWHA described "being taken care of" (especially in prisons) in terms of eligibility for care, yet this facilitated engagement in care for some more than others. Factors such as segregated housing of HIV positive inmates, discrete access to medical care, and a sense of safety in terms of privacy facilitated care engagement for older AAPLWHA. This echoes previous research on factors that facilitate engagement in the HIV care cascade before, during and after incarceration (Dennis, Barrington, Hino, Gould et al., 2015; Harawa et al., 2008; Iroh et al., 2015). Participants' experiences reflected the complete range of the HIV care cascade framework. Some older AAPLWHA were diagnosed in incarceration settings and were able to access services, which they would have struggled to receive outside of the correctional setting. Others were often for the first time successfully retained in care and able to achieve viral suppression while incarcerated.

However, for some participants the incarceration experience, lead to disengagement in care through the behavioral processes of "keeping low", of "isolating self", of "avoiding care," and "not disclosing status." A surprising finding within the behavioral processes was non-disclosure at the cost of not receiving HIV care. Two participants who were aware of their HIV diagnosis decided not to disclose their HIV status upon entering the correctional system due to fear of stigma and discrimination. At the time of their incarceration both participants were younger (less than 50 years) and 
attributed not disclosing their HIV status and subsequent disengagement in care to their younger age and the time (1990s and pre HAART) their incarceration began. The decision to disengage in care was particularly interesting when participants were aware of the imminent health risks related to their decision. This finding aligns with Zawitz and colleagues (2004) who note that incarcerated PLWHA fear HIV stigma more than disease progression or even death. This was most often true for participants incarcerated in a jail. Older AAPLWHA described counting the cost of facing stigma and discrimination versus taking a chance on getting sick or not receiving HIV medication while incarcerated.

Psychological processes (Figure 7.2) were temporal and focused on past, present and anticipation - future. The most prominent issues for participants after release was the ability to negotiate stigmatizing perceptions of formerly incarcerated older AAPLWHA by the general public, employers, health care providers and communities. Another prevalent concern was a lack of knowledge on how to obtain care once released, as well as fear of loosing access to care post-release. There was a lack of connecting participants to medical care and ASOs once they were released even if policies and programs to assist older AAPLWHAs were in place, such as housing, substance abuse programs, mental health services, and job support. This finding highlights the need for enhanced relationships between correctional facilities and local ASOs in order to prevent disengagement in care. A stronger collaboration among agencies might have prevented participants from becoming disengaged from HIV care post-incarceration. The findings highlight a gap between post-release and reengagement in care because participants often lost access to health care post release and were unaware of how to secure health insurance. This aligns with Ammon and colleagues, (2018) findings that HIV care engagement post-jail release in PLWHA is often lower than before incarceration, and 
suggests that an improved HIV care continuity for individuals incarcerated in jails is needed.

Two coping strategies within the temporal process "focusing on self" and "learning to develop protective behaviors" were identified. The second strategy was linked to present coping as well as anticipating the future by predicting tactics one planned to engage in post incarceration. Some participants who stayed successfully engaged in care were able to access resources with the help of an attentive physician knowledgeable of local ASOs and existing resources. Based on these results, it appears that older AAPLWHA are most in need of consistent linkage or (re)linkage to care in their communities post-release. Greater collaboration between correctional facilities and local ASOs also offers a significant benefit to participants post-release.

The second goal of the study was to develop an inductive theory of the process related to incarceration experiences among older AAPLWHA and their engagement in care. The inductive theory "Older AAPLWHAs Journey towards Engagement in Care during Incarceration" (Figure 8.1) reflects a process and explains through four phases how older AAPLWHA engaged in the process of HIV care during their incarceration experiences. The transition through the four phases 1) Entering the correctional system, 2) Developing Understandings of HIV within the correctional system, 3) Engagement in HIV care, and 4) Linkage to care post-release was gradual or occurred multiple times, such as in the case of multiple incarcerations. The inductive theory provides information on the lived incarceration experiences of older AAPLWHA, adds to the existing literature about incarceration, older AAs and HIV, and provides unique insights in the processes older AAPLWHA engage in. It also accounts for the internal and external factors that affected participants' engagement in care, such as stigma, race, the correctional setting, and time. The presented inductive theory "Older AAPLWHAs Journey towards 
Engagement in Care during Incarceration" builds on the knowledge of the HIV care cascade and adds to the understandings of the complex role that incarceration experiences play for older AAPLWHA in their HIV care continuum and how to better support individuals in achieving optimal health outcomes.

The HIV care cascade (Gardener, McLees, Steiner, Del Rio et al., 2011; U.S. Department of Health and Human Services, 2013; Whiteside, Cohen, Bradley, Skarbinski et al., 2014) (see Figure 9.1) is a public health framework that identifies the stages of HIV treatment and ranges from diagnosis to achieving an undetectable viral load through consistent retention in care and adherence to ART regimens. The older AAPLWHA in this study encountered multiple or all the stages of the continuum during their incarceration. Some participants moved both backwards and forwards through specific stages throughout their incarceration experiences.

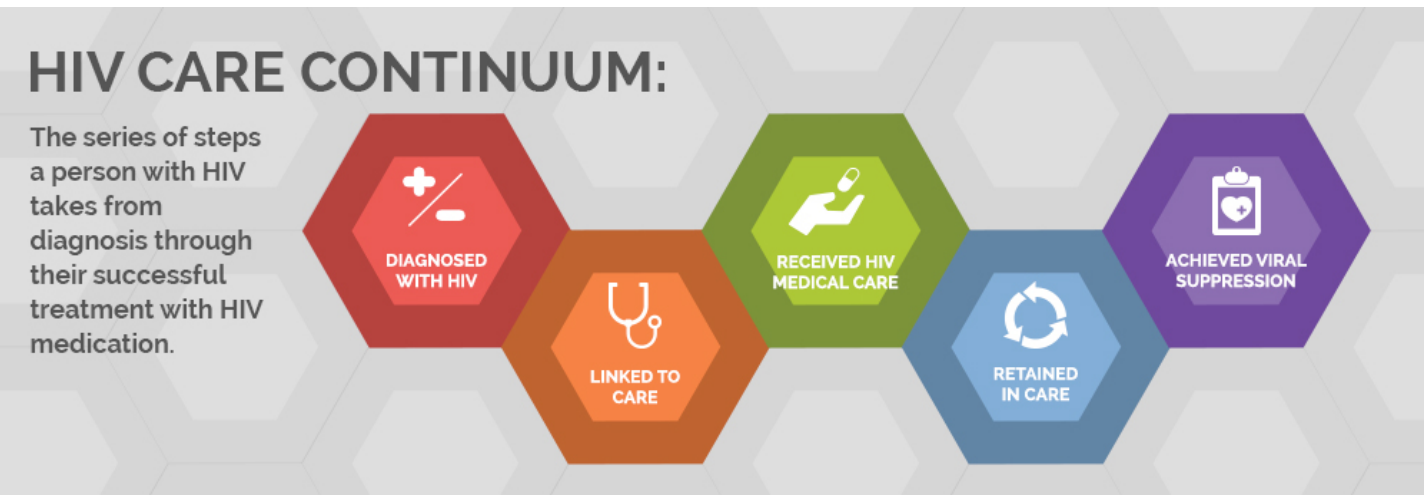

Figure 9.1. The HIV Treatment Cascade from HIV.gov

The presented inductive theory uniquely addresses older AAPLWHAs journey towards engagement in care and uncovered inadequacies and inequalities in access to HIV care services, as well as the great potential of care engagement for this population while incarcerated. Addressing “Older AAPLWHAs Journey towards Engagement in Care during Incarceration" has the potential to create much needed interventions (i.e. 
developing programs aimed at improving linkage to care post-release) for older AAPLWHA who are or have been incarcerated in the past. In the context of program and policy development, the study findings can provide insights regarding the needs of this underserved population. ASOs, correctional facilities and health care providers can use these findings to guide improvements of the HIV care cascade for older incarcerated AAPLWHAs and post-incarceration continuity of care.

\section{Limitations and Suggestions for Future Research}

Some limitations of this study should be noted. First, the 22 interviews drawn from the original study $(\mathrm{n}=35)$ examining stress, stigma and engagement in HIV care in Louisville were not solely focused on the impact of incarceration experiences. Thus, the seven follow-up interviews from the theoretical sample focused exclusively on the topic of incarceration. Ideally, the incarceration experience itself would have been a primary focus of the initial study, but the research team was not prepared for the large percentage (over $60 \%$ ) of the sample who had incarceration experiences. Therefore, questions about the incarceration experience were not built into the initial interview guide, but mentioned to varying extents in each interview, and through the collection of demographic information. Because incarceration experiences were brought up to varying extents during the original data collection, the inductive theory relies heavily on the experiences of the theoretical sample $(\mathrm{N}=7)$. Future studies would benefit from a larger sample to further develop the theory. Another limitation to note was recall bias. Participants' past incarceration experiences ranged from two to twenty years at the time of the interviews. Therefore, some of the participants' narratives do not reflect the current reintegration practices in Louisville and are not transferable to other settings across the United States. Different cities have vastly different post-incarceration services available to persons leaving the correctional environment. However, there is value in exploring these context 
specific and historical accounts of incarceration experiences to learn how to best meet the needs and trauma experiences of this population in post-release care. The data for this study was collected in 2016 and reflects the context of older AAPLWHA at this point in time. Future research is needed to study this population in the context of the COVID-19 pandemic and explore how the current tensions between law enforcement and the AA community contribute and perpetuate race-based trauma in this population.

\section{Conclusion}

Older AAPLWHA with incarceration histories are a vulnerable population and face a myriad of challenges. Yet limited research portrays their lived incarceration experiences. This study directly attended to this issue by understanding the processes older AA adults experience related to their incarceration and engagement in care. Grounded in older AAPLWHAs experiences, this study established an inductive theory, titled "Older AAPLWHAs Journey towards Engagement in Care during Incarceration." Through this inductive theory we come to better understand the complexity of their lived experiences, their challenges as they develop understandings of what it means to live with HIV in the correctional system and the barriers and facilitators to HIV care engagement. 


\section{REFERENCES}

ACLU and Human Rights Watch Report calls on South Carolina and Alabama to stop segregating prisoners with HIV (2010). Conditions in HIV Units Cruel, Inhuman and Degrading. Accessed at: https://www.aclu.org/news/aclu-and-human-rightswatch-report-calls-south-carolina-and-alabama-stop-segregatingprisoners?redirect=hiv-aids-prisoners-rights/aclu-and-human-rights-watch-reportcalls-south-carolina-and-alabama-stop-s

Aday, R. H., Krabill, J.J. (2012). Older and geriatric offenders: critical issues for the $21^{\text {st }}$ century. Special needs offenders in correctional institutions, 1, 203-233.

Aday, R. H., (2003). Aging prisoners: Crisis in American Corrections. Penn State Press.

AIDSinfonet (2014). HIV and Prison and Jails. Accessed at http://www.aidsinfonet.org/fact_sheets/view/615\#HIV_and_Correct

Alarid, L. F., \& Marquart, J. W. (2009). Officer perceptions of risk of contracting HIV/AIDS in prison: A two-state-comparison. The Prison Journal, 89(4), 440459.

Altice, F. L., Marinovich, A., Khoshnood, K., Blankenship, K. M., Springer, S. A., \& Selwyn, P. A. (2005). Correlates of HIV infection among incarcerated women: implications for improving detection of HIV infection. Journal of Urban Health, 82(2), 312-326.

Althoff, A. L., Zelenev, A., Meyer, J.P., Fu, J., Brown, S. E., Vagenas, P.,...\& Altice, F. L. (2013). Correlates of retention in HIV care after release from jail: results from a multi-site study. AIDS and Behavior, 17(2), 156-170.

Ammon, B., Iroh, P., Tiruneh, Y., Li, X., Montague, B. T., Rich, J. D., \& Nijhawan, A. E. (2018). HIV care after jail: low rates of engagement in a vulnerable population. Journal of Urban Health, 95(4), 488-498.

Andrinopoulos, K., Kerrigan, D., Figueroa, J. P., Reese, R., Gaydos, C. A., Bennett, L., ... \& Ellen, J. M. (2010). Establishment of an HIV/sexually transmitted disease programme and prevalence of infection among incarcerated men in Jamaica. International journal of STD \& AIDS, 21(2), 114-119.

Arksey, H., \& O'Malley, L. (2005). Scoping studies: towards a methodological framework. International journal of social research methodology, 8(1), 19-32.

Åsbring, P., \& Närvänen, A. L. (2002). Women's experiences of stigma in relation to chronic fatigue syndrome and fibromyalgia. Qualitative health research, 12(2), 148-160. 
Bakken, S., Holzemer, W. L., Brown, M. A., Powell-Cope, G. M., Turner, J. G., Inouye, J., Nokes, K. M., ... Corless, I. B. (2000). Relationships between perception of engagement with health care provider and demographic characteristics, health status, and adherence to therapeutic regimen in persons with HIV/AIDS. AIDS Patient Care and STDs, 14(4), 189-97.

Baumer, E. P. (2013). Reassessing and redirecting research on race and sentencing. Justice Quarterly, 30(2), 231-261.

Beckwith, C. G., Zaller, N. D., Fu, J. J., Montague, B. T., \& Rich, J. D. (2010). Opportunities to diagnose, treat, and prevent HIV in the criminal justice system. Journal of acquired immune deficiency syndromes (1999), 55(Suppl 1), S49.

Beckwith, C. G., Nunn, A., Baucom, S., Getachew, A., Akinwumi, A., Herdman, B., ... \& Kuo, I. (2012). Rapid HIV testing in large urban jails. American journal of public health, 102(S2), S184-S186.

Berger, B. E., Ferrans, C. E., \& Lashley, F. R. (2001). Measuring stigma in people with HIV: Psychometric assessment of the HIV stigma scale. Research in Nursing \& Health, 24(6), 518-529.

Blumer, H. (1954). What is wrong with social theory? American Sociological Review, 19, 3-10. Doi: $10.2307 / 2088165$

Blumer, H. (1969). Symbolic Interactionism: Perspective and method. Berkely.

Braithwaite, R. L., \& Arriola, K. R. (2003). Male prisoners and HIV prevention: a call for action ignored. American Journal of Public Health, 93(5), 759-763.

Braveman, P., \& Gruskin, S. (2003). Defining equity in health. Journal of epidemiology and community health, 57(4), 254-258.

Brennan, D. J., Emlet, C. A., \& Eady, A. (2011). HIV, sexual health, and psychosocial issues among older adults living with HIV in North America. Ageing International, 36(3), 313-333.

Brinkley-Rubinstein, L., \& Turner, W. L. (2013). Health impact of incarceration on HIVpositive African American males: a qualitative exploration. AIDS patient care and STDs, 27(8), 450-458.

Brothers, T. D., Kirkland, S., Guaraldi, G., Falutz, J., Theou, O., Johnston, B. L., \& Rockwood, K. (2014). Frailty in people aging with human immunodeficiency virus (HIV) infection. Journal of Infectious Diseases, jiu258.

Capeau, J. (2011). Premature aging and premature age-related comorbidities in HIVinfected patients: facts and hypotheses. Clinical infectious diseases, cir628.

Carsen, E. A. (2014). Prisoners in 2013. Bureau of Justice Statistics, U.S. Department of Justice. http://www.bjs.gov/content/pub/pdf/p13.pdf. 
Center for Disease Control and Prevention (2013). Diagnoses of HIV infection among adults aged 50 years and older in the United States and dependent areas, 20072010. HIV Surveillance Supplemental Report, 18(3), 1 - 70. Retrieved from http://www.cdc.gov/hiv/pdf/statistics_2010_HIV_Surveillance_Report_vol_18_no 3.pdf CDC, 2013

Centers for Disease Control and Prevention (2016). CDC Fact Sheet Todays HIV/AIDS Epidemic Accessed at:

https:/www.cdc.gov/nchhstp/newsroom/docs/factsheets/todaysepidemic-508.pdf

Centers for Disease Control and Prevention (2018). HIV Surveillance Report

(Preliminary); vol. 30. http://www.cdc.gov/hiv/library/reports/hivsurveillance.html

Centers for Disease Control and Prevention (2019). Diagnoses of HIV Infection in the United States and dependent areas. HIV Surveillance Report 2019. Accessed at: https://www.cdc.gov/hiv/library/reports/hiv-surveillance.html

Center for HIV Law and Policy, (2017). http://hivlawandpolicy.org/

Charmaz, K., Denzin, N. K., \& Lincoln, Y. S. (2003). Strategies of qualitative inquiry. Strategies of qualitative inquiry.

Charmaz, K. (2014). Constructing grounded theory. Sage.

Chettiar, I. M., Bunting, W. C., \& Schotter, G. (2012). At America's expense: The mass incarceration of the elderly.

Clair, S., Singer, M., Huertas, E., \& Weeks, M. (2003). Unintended consequences of using an oral HIV test on HIV knowledge. AIDS care, 15(4), 575-580.

Clarke, A. E. (2003). Situational analyses: Grounded theory mapping after the postmodern turn. Symbolic interaction, 26(4), 553-576.

Clarke, A. (2005). Situational analysis: Grounded theory after the postmodern turn. Sage.

Clarke, A. (2009). Situational analysis. Innovations in Social Science Research Methods1, 55 .

Clarke, A. E., Friese, C., \& Washburn, R. (Eds.). (2015). Situational analysis in practice: Mapping research with grounded theory (Vol. 1). Left Coast Press.

Clemmitt, M. (2007). Prison health care. CQ Press.

Cohen, J. (1960). A coefficient of agreement for nominal scales. Educational and psychological measurement, 20(1), 37-46.

Cohen, S., Kamarck, T., \& Mermelstein, R. (1994). Perceived stress scale. Measuring stress: A guide for health and social scientists, 10, 1-2.

Collins, P. H., Bilge, S. (2020). Intersectionality. John Wiley \& Sons. 
Commonwealth of Kentucky Department of Corrections (2018). Chapter 13: Medical and Health Care Services. https://corrections.ky.gov/About/cpp/Pages/Chapter13.aspx

Corbin, J., \& Strauss, A. (2008). Basics of qualitative research: Techniques and procedures for developing grounded theory.

Creswell, J. W., \& Poth, C. N. (2016). Qualitative inquiry and research design: Choosing among five approaches. Sage publications.

Creswell, J. W., \& Miller, D. L. (2000). Determining validity in qualitative inquiry. Theory into practice, 39(3),124-130.

Dalmida, S. G., McDougal Jr, Mugoya, G.C., Foster, P. P.Plyman, M., \& Burrage, J. (2018). Engagement of African Americans with rapid HIVtesting and HIV care. HIV/AIDS research and treatment: open journal,2018, S38.

Dedoose Software Version, D. 4.5, web application for managing, analyzing, and presenting qualitative and mixed method research data (2013). Los Angeles, CA: SocioCultural Research Consultants, LLC (www. dedoose. com).

Dennis, A. C., Barrington, C., Hino, S., Gould, M., Wohl, D., \& Golin, C. E. (2015). "You're in a world of chaos": experiences accessing HIV care and adhering to medications after incarceration. Journal of the Association of Nurses in AIDS Care, 16(5), 542-555.

Denzin, N. K., \& Lincoln, Y. S. (Eds.). (2011). The Sage handbook of qualitative research.

Dolan, K., Wirtz, A. L., Moazen, B., Ndeffo-mbah, M., Galvani, A., Kinner, S. A., ... \& Hellard, M. (2016). Global burden of HIV, viral hepatitis, and tuberculosis in prisoners and detainees. The Lancet, 388(10049), 1089-1102.

Dwyer, S. C., \& Buckle, J. L. (2009). The space between: On being an insider-outsider in qualitative research. International journal of qualitative methods, 8(1), 54-63.

Earnshaw, V. A., \& Chaudoir, S. R. (2009). From conceptualizing to measuring HIV stigma: a review of HIV stigma mechanism measures. AIDS and Behavior, 13(6), 1160 .

Earnshaw, V. A., Smith, L.R., Chaudoir, S. R, Amico, K. R., \& Copenhaver, M. M. (2013). HIV stigma mechanisms and well-being among PLWH: a test of the HIV stigma framework. AIDS and Behavior, 17(5), 1785-1795.

Egger, M., May, M., Chene, G., Phillips, A. N. Ledergerber, B., Dabis, F.,.. \& \& Lundgren, J. D. (2002). Prognosis of HIV-1 infected patients starting highly active antiretroviral therapy: a collaborative analysis of prospective studies. The Lancet, 360(9327), 119-129.

Emlet, C. A. (2006a). An examination of the social networks and social isolation in older and younger adults living with HIV/AIDS. Health \& social work, 31(4), 299-308. 
Emlet, C. A. (2006). "You're awfully old to have this disease": Experiences of stigma and ageism in adults 50 years and older living with HIV/AIDS. The Gerontologist, 46(6), 781-790.

Emlet, C. A., Fredriksen-Goldsen, K. I., \& Kim, H. J. (2013). Risk and protective factors associated with health-related quality of life among older gay and bisexual men living with HIV disease. The Gerontologist, 53(6), 963-972.

Emlet, C. A. (2014). Current knowledge and future directions on aging and HIV research. Behavioral Medicine, 40(3), 143-148.

Emlet, C. A., Harris, L., Furlotte, C., Brennan, D. J., \& Pierpaoli, C. M. (2017). 'I'm happy in my life now, I'm a positive person': approaches to successful ageing in older adults living with HIV in Ontario, Canada. Ageing and Society, 37(10), 2128

Erlandson, D. A. (1993). Doing naturalistic inquiry: A guide to methods. Sage.

Falter, R. G. (2006). Elderly inmates: An emerging correctional population. Correctional Health Journal, 1, 52-69.

Fazel S, Baillargeon J. (2011). The health of prisoners. Lancet; 377: 956-65.

Fernald, D. H., \& Duclos, C. W. (2005). Enhance Your Team-Based Qualitative Research. Annals of Family Medicine, 3(4), 360-364. http://doi.org/10.1370/afm.290

Fogel, C. I., Crandell, J. L., Neevel, A. M., Parker, S. D., Carry, M., White, B. L., ... \& Gelaude, D. J. (2015). Efficacy of an adapted HIV and sexually transmitted infection prevention intervention for incarcerated women: a randomized controlled trial. American journal of public health, 105(4), 802-809.

Folkman, S., \& Lazarus, R. S. (1984). Stress, appraisal, and coping (pp.150-153). New York: Springer Publishing Company.

Fredriksen-Goldsen, K. I., Kim, H. J., Emlet, C. A., Muraco, A., Erosheva, E. A., HoyEllis, C. P., ... \& Petry, H. (2011). The aging and health report: Disparities and resilience among lesbian, gay, bisexual, and transgender older adults. Seattle: Institute for Multigenerational Health.

Gardner, E. M., McLees, M. P., Steiner, J. F., Del Rio, C., Burman, W. J. (2011). The spectrum of engagement in HIV care and its relevance to test-and-treat strategies for prevention of HIV infection. Clinical infectious diseases, 52(6), 793-800.

Ghidei, L., Simone, M. J., Salow, M. J., Zimmerman, K. M., Paquin, A. M., Skarf, L. M., ... \& Rudolph, J. L. (2013). Aging, antiretrovirals, and adherence: a meta analysis of adherence among older HIV-infected individuals. Drugs \& aging, 30(10), 809819.

Glaser, B. G. (1978). Theoretical sensitivity: Advances in the methodology of grounded theory. Sociology Pr. 
Glaser, B. G. (1998). Doing grounded theory: Issues and discussions. Sociology Press.

Glaser, B. G. (2001). The grounded theory perspective: Conceptualization contrasted with description. Sociology Press.

Glaser, B. G. (2003). The grounded theory perspective II: Descriptions remodeling of grounded theory methodology. Sociology Press.

Glaser, B. G. (2005). The grounded theory perspective III: Theoretical coding. Sociology Press.

Glaser, B. G. (2011). Getting out of the data: Grounded theory conceptualization. Sociology press.

Glaser, B., \& Strauss, A. (1967). The discovery of grounded theory. Weidenfield \& Nicolson, London, 1-19.

Guest, G., \& MacQueen, K. M. (Eds.). (2007). Handbook for team-based qualitative research. Rowman Altamira.

Hammett, T. M. (2006). HIV/AIDS and other infectious diseases among correctional inmates: transmission, burden, and an appropriate response. American Journal of Public Health, 96(6), 974-978.

Harawa, N. T., Bingham, T. A., Butler, Q. R., Dalton, K. S., Cunningham, W. E., Behel, S., \& MacKellar, D. A. (2009). Using arrest charge to screen for undiagnosed HIV infection among new arrestees: a study in Los Angeles County. Journal of Correctional Health Care, 15(2), 105-117. doi:10.1177/1078345808330038

Harris, L.M., Emlet, C.A., Pierpaoli Parker, C., \& Furlotte, C. (2018). Timing of diagnosis: Understanding resilience narratives of HIV positive older adults diagnosed pre-and post-HAART. Journal of gerontological social work (61)1, 78103.

Harris, L.M., Crawford, T.N., Kerr, J., Thomas, T.A. \& Schmidt, V. (2020). African American Older Adults Living with HIV: Exploring Stress, Stigma and Engagement in HIV Care. Journal of Health Care for the Poor and Underserved.

Hayes, A. J., Burns, A., Turnbull, P., \& Shaw, J. J. (2012). The health and social needs of older male prisoners. International journal of geriatric psychiatry, 27(11), 11551162 .

Heckman, T. G., Heckman, B. D., Kochman, A., Sikkema, K. J., Suhr, J., \& Goodkin, K. (2002). Psychological symptoms among persons 50 years of age and older living with HIV disease. Aging \& mental health, 6(2), 121-128.

Heckman, T. G., \& Halkitis, P. N. (2014). Biopsychosocial aspects of HIV and aging.

Hesse-Biber, S. N., \& Leavy, P. (2010). The practice of qualitative research. Sage. 
High, K. P., Brennan-Ing, M., Clifford, D. B., Cohen, M. H., Currier, J., Deeks, S. G., ... \& Volberding, P. (2012). HIV and Aging: State of knowledge and areas of critical need for research. A report to the NIH Office of AIDS Research by the HIV and Aging Working Group. Journal of acquired immune deficiency syndromes (1999), 60 (Suppl 1), S1-18.

Homer, J., \& Milstein, B. (2002, July). Communities with multiple afflictions: a system dynamics approach to the study and prevention of syndemics. In 20th International Conference of the System Dynamics Society.

HIV/AIDS Care Continuum: U.S. Department of Health and Human Services. 2013. Available from https//www.hiv.gov/federal-response/policies-issues/hiv-aids-carecontinuum

Huberman, A. M., \& Miles, M. B. (1994). Data management and analysis methods.

Hudson, A. L., Nyamathi, A., Greengold, B., Slagle, A., Koniak-Griffin, D., Khalilifard, F., \&Getzoff, D. (2010). Health-seeking challenges among homeless youth. Nursing research, 59(3), 212.

Inciardi, J. A. (1996). HIV risk reduction and service delivery strategies in criminal justice settings. Journal of Substance Abuse Treatment, 13(5), 421-428.

Iroh, P. A., Mayo, H., \& Nijhawan, A. E. (2015). The HIV care cascade before, during, and after incarceration: a systematic review and data synthesis. American journal of public health, 105(7), e5-e16.

James-Borga, J. C. (2013). Living with hiv disease: A phenomenological study of a group of older black/African American women. City University of New York.

Johnson, R. C., \& Raphael, S. (2009). The effects of male incarceration dynamics on acquired immune deficiency syndrome infection rates among African American women and men. The Journal of Law and Economics, 52(2), 251-293.

Jolly, D. H., Mueller, M. P., Chen, M., Alston, L. M., Hawley, M., Okumu, E., ... \& MacQueen, K. M. (2016). Concurrency and other sexual risk behaviors among black young adults in a southeastern city. AIDS Education and Prevention, 28(1), 59-76.

Juergens, R., Nowak, M., \& Day, M. (2011). HIV and incarceration: prisons and detention. Journal of the International AIDS Society, 14(1), 26.

Justice, A. C. (2010). HIV and aging: time for a new paradigm. Current HIV/AIDS Reports, 7(2), 69-76.

Kentucky Department of Corrections (2017). Accessed at: http://corrections.ky.gov/Pages/default.aspx

Kerstetter, K. (2012). Insider, Outsider, or somewhere in between: The impact of researchers' identities on the community-based research process. Journal of Rural Social Sciences, 27(2), 99. 
Khan, M. R., Golin, C. E., Friedman, S. R., Scheidell, J. D., Adimora, A. A., JudonMonk, S., ... \& Myers, D. (2015). STI/HIV sexual risk behavior and prevalent STI among incarcerated African American men in committed partnerships: the significance of poverty, mood disorders, and substance use. AIDS and Behavior, 19(8), 1478-1490.

Krebs, C. P., \& Simmons, M. (2002). Intraprison HIV transmission: an assessment of whether it occurs, how it occurs, and who is at risk. AIDS education and prevention, 14(5 Supplement), 53-64.

Krebs, C. P. (2006). Inmate factors associated with HIV transmission in prison. Criminology \& Public Policy, 5(1), 113-135.

Lazarus, R. S. (1993). Coping theory and research: Past, present, and future. Fifty years of the research and theory of $R S$ Lazarus: An analysis of historical and perennial issues, 336-338.

Laurencin, C. T., Murdock, C. J., Laurencin, L., \& Christensen, D. M. (2018). HIV/AIDS and the African-American community 2018: a decade call to action. Journal of racial and ethnic health disparities, 5(3), 449-458.

Levy, M. E., Wilton, L., Phillips, G., Glick, S. N., Kuo, I., Brewer, R. A., ... \& Magnus, M. (2014). Understanding structural barriers to accessing HIV testing and prevention services among black men who have sex with men (BMSM) in the United States. AIDS and Behavior, 18(5), 972-996.

Lincoln, Y. S., \& Guba, E. G. (1985). Naturalistic inquiry (vol. 75).

Link, B. G., Wells, J., Phelan, J. C., \& Yang, L. (2015). Understanding the importance of "symbolic interaction stigma": How expectations about the reactions of others adds to the burden of mental illness stigma. Psychiatric rehabilitation journal, 38(2), 117.

Linley, L., Prejean, J., An, Q., Chen, M., \& Hall, H. I. (2012). Racial/ethnic disparities in HIV diagnoses among persons aged 50 years and older in 37 US States, 20052008. American journal of public health, 102(8), 1527-1534.

Lyons, T., Osunkoya, E., Anguh, I., Adefuye, A., \& Balogun, J. (2014). HIV prevention and education in state prison systems: an update. Journal of Correctional Health Care, 20(2), 105-115.

MacQueen, K. M., Chen, M., Jolly, D., Mueller, M. P., Okumu, E., Eley, N. T., ... \& Rogers, R. C. (2015). HIV testing experience and risk behavior among sexually active black young adults: a CBPR-based study using respondent-driven sampling in Durham, North Carolina. American journal of community psychology, 55(3-4), 433-443.

Mahajan, A. P., Sayles, J. N., Patel, V. A., Remien, R. H., Ortiz, D., Szekeres, G., \& Coates, T. J. (2008). Stigma in the HIV/AIDS epidemic: a review of the literature and recommendations for the way forward. AIDS (London, England), 22(Suppl 2), S67. 
Martin, V. B. (2006). The Postmodern Turn: Shall classic grounded theory take that detour? A review essay. The Grounded Theory Review: An International Journal, 5(2/3), 119-129.

Martin, J., \& Volberding, P. (2010). HIV and premature aging: A field still in its infancy. Annals of internal medicine, 153(7), 477-479.

Martos, A. J., Valera, P., Bockting, W. O., \& Wilson, P. A. (2016). Exploring sexual health among young Black men who have sex with men in New York City. Health Education Research, 31(3), 295-313.

Maruschak, L. M., \& Beavers, R. (2009). HIV in Prisons, 2007-08. Bureau of justice statistics bulletin, 1-12.

Maruschak, L. M. (2012). Revised 2015. HIV in prisons , 2001 - 2010 (Bulletin No. NCJ 238877). U.S. Department of Justice, Bureau of Justice Statistics.

Mason, J. (2002). Sampling and selection in qualitative research. Qualitative researching. Sage Publications.

Mauer, M. (2011). Addressing racial disparities in incarceration. The Prison Journal, 91(3_suppl), 87S-101S.

Mauer, M. \& King, R.S. (2007). Uneven justice: State rates of incarceration by race and ethnicity.

May, J. P., \& Williams Jr, E. L. (2002). Acceptability of condom availability in a US jail. AIDS Education and Prevention, 14(5 Supplement), 85-91.

Mayer, K. H., Spaulding, A., Stephenson, B., Macalino, G., Ruby, W., Clarke, J. G., \& Flanigan, T. P. (2002). Human immunodeficiency virus in correctional facilities: a review. Clinical Infectious Diseases, 35(3), 305-312.

Merriam, S. B. (1998). Qualitative Research and Case Study Applications in Education. Revised and Expanded from "Case Study Research in Education." Jossey-Bass Publishers, 350 Sansome St, San Francisco, CA 94104.

Merriam, S. B., \& Tisdell, E. J. (2015). Qualitative research: A guide to design and implementation. John Wiley \& Sons.

Mertens, D. (1998). Research methods in education and psychology: Integrating diversity with quantitative and qualitative approaches. Thousand Oaks: Sage.

Meyer, J. P., Cepeda, J., Taxman, F. S., \& Altice, F. L. (2015). Sex-related disparities in criminal justice and HIV treatment outcomes: a retrospective cohort study of HIV infected inmates. American journal of public health, 105(9), 1901-1910.

Meyer, J. P., Cepeda, J., Wu, J., Trestman, R. L., Altice, F. L., \& Springer, S. A. (2014). Optimization of human immunodeficiency virus treatment during incarceration: viral suppression at the prison gate. JAMA internal medicine, 174(5), 721-729. 
McCarthy, E., Myers, J. J., Reeves, K., \& Zack, B. (2016). Understanding the Syndemic Connections Between HIV and Incarceration Among African American Men, Especially African American Men Who Have Sex with Men. In Understanding the HIV/AIDS Epidemic in the United States (pp. 217-240). Springer International Publishing.

McClelland, G. M., Teplin, L. A., Abram, K. M., \& Jacobs, N. (2002). HIV and AIDS risk behaviors among female jail detainees: implications for public health policy. American Journal of Public Health, 92(5), 818-825.

Miles, M. B., \& Huberman, A. M. (1994). Qualitative data analysis: An expanded sourcebook. sage.

Miles, M. B., Huberman, A. M., \& Saldana, J. (2013). Qualitative data analysis. Sage.

Minton, T. D., \& Golinelli, D. (2014). Jail Inmates at Midyear 2013-statistical Tables. Washington, DC: US Department of Justice, Office of Justice Programs, Bureau of Justice Statistics.

Nogueras, M., Navarro, G., Anton, E., Sala, M., Cervantes, M., Amengual, M., \& Segura, F. (2006). Epidemiological and clinical features, response to HAART, and survival in HIV-infected patients diagnosed at the age of 50 or more. $B M C$ infectious diseases, 6(1), 1-9.

Nowotny, K., Cepeda, A., James-Hawkins, L., \& Boardman, J. (2016). Growing old behind bars. Journal of Aging and Health, 28(6), 935-956. doi:10.1177/0898264315614007

Nyamathi, A. (1989). Comprehensive health seeking and coping paradigm. Journal of Advanced Nursing, 14(4), 281-290.

Nyamathi, A (1992). Comparative study of factors relating to HIV risk level of black homeless women. JAIDS Journal of Acquired Immune Deficiency Syndromes, 5(3), 222-228.

Ormston, R., Spencer, L., Barnard, M., \& Snape, D. (2014). The foundations of qualitative research. Qualitative research practice: A guide for social science students and researchers, 2.

Padgett, D. (2004). The qualitative research experience. Wadsworth/Thomson Learning.

Park, C. L., \& Folkman, S. (1997). Meaning in the context of stress and coping. Review of general psychology, 1(2), 115-144.

Parker, R., \& Aggleton, P. (2003). HIV and AIDS-related stigma and discrimination: a conceptual framework and implications for action. Social science \& medicine, $57(1), 13-24$.

Pathai, S., Bajillan, H., Landay, A. L., \& High, K. P. (2013). Is HIV a model of accelerated or accentuated aging?. The Journals of Gerontology Series A: Biological Sciences and Medical Sciences, glt168. 
Patton, M. Q. (2002). Two decades of developments in qualitative inquiry: A personal, experiential perspective. Qualitative social work, 1(3), 261-283.

Plattner, I. E., \& Meiring, N. (2006). Living with HIV: The psychological relevance of meaning making. AIDS care 18(3), 241-245.

Polonsky, S., Kerr, S., Harris, B., Gaiter, J., Fichtner, R. R., \& Kennedy, M. G. (1994). HIV prevention in prisons and jails: obstacles and opportunities. Public health reports, 109(5), 615 .

Pope, J. L. (2009). HIV testing in state correctional systems. JL \& Health, $22,17$.

Raphael, D. (2009). Social determinants of health: Canadian perspectives. Canadian Scholars' Press.

Rich, J. D., DiClemente, R., Levy, J., Lyda, K., Ruiz, M., Rosen, D. L., \& Dumont, D. (2013). Correctional facilities as partners in reducing HIV disparities. Journal of acquired immune deficiency syndromes (1999), 63(0 1), S49.

Rowell-Cunsolo, T.L., El-Bassel, N., \& Hart, C.L. (2016). Black Americans and incarceration: a neglected public health opportunity fir HIV risk reduction. Journal of health care for the poor and underserved, 27(1),114.

Roe, J., Joseph, S., \& Middleton, H. (2010). a theoretical approach to understanding stigma and recovey. Mental Health Review Journal, 15(1).

Rosen, D. L., Schoenbach, V. J., Wohl, D. A., White, B. L., Stewart, P. W., \& Golin, C. E. (2009a). An evaluation of HIV testing among inmates in the North Carolina prison system. American journal of public health, 99(S2), S452-S459.

Rosen, D. L., Schoenbach, V. J., Wohl, D. A., White, B. L., Stewart, P. W., \& Golin, C. E. (2009b). Characteristics and behaviors associated with HIV infection among inmates in the North Carolina prison system. American journal of public health, 99(6), 1123-1130.

Rumrill, P. D., Fitzgerald, S. M., \& Merchant, W. R. (2010). Using scoping literature reviews as a means of understanding and interpreting existing literature. Work (Reading, Mass.), 35(3), 399-404.

Sawyer, W., \& Wagner, P. (2019). Mass incarceration: The whole pie 2019. Prison Policy Initiative, 19.

Schlotfeldt, R. (1981). Nursing in the future. Nursing Outlook, 29, 295-301.

Sheiham, A. (2009). Closing the gap in a generation: health equity through action on the social determinants of health. A report of the WHO Commission on Social Determinants of Health (CSDH) 2008. Community Dent Health, 26(1), 2-3.

Shilo, G., \& Mor, Z. (2015). Seeking sex online: social and sexual risk factors among adolescent and young gay and bisexual men. Sexual health, 12(3), 217-223. 
Shippy, R. A., \& Karpiak, S. E. (2005). The aging HIV/AIDS population: fragile social networks. Aging \& mental health, 9(3), 246-254.

Singer, M., \& Clair, S. (2003). Syndemics and public health: Reconceptualizing disease in bio-social context. Medical anthropology quarterly, 17(4), 423-441.

Small, W., Wood, E., Betteridge, G., Montaner, J., \& Kerr, T. (2009). The impact of incarceration upon adherence to HIV treatment among HIV-positive injection drug users: a qualitative study. AIDS care, 21(6), 708-714.

Sprague, C., Scanlon, M. L., Radhakrishnan, B., \& Pantalone, D. W. (2016). The HIV Prison Paradox Agency and HIV-Positive Women's Experiences in Jail and Prison in Alabama. Qualitative Health Research, 1049732316672640.

Springer, S. A., Pesanti, E., Hodges, J., Macura, T., Doros, G., \& Altice, F. L. (2004). Effectiveness of antiretroviral therapy among HIV-infected prisoners: reincarceration and the lack of sustained benefit after release to the community. Clinical Infectious Diseases, 38(12), 1754-1760.

Stock, M. L., Gibbons, F. X., Peterson, L. M., \& Gerrard, M. (2013). The effects of racial discrimination on the HIV-risk cognitions and behaviors of Black adolescents and young adults. Health Psychology, 32(5), 543.

Strauss, A., \& Corbin, J. (1990). Basics of qualitative research (Vol. 15). Newbury Park, CA: Sage.

Strauss, A., \& Corbin, J. (1998). Basics of qualitative research: Procedures and techniques for developing grounded theory.

Tietz, D. (2013). Testimony before the Senate Special Committee on Aging, Older Americans, the changing face of HIV/AIDS in America. Accessed at: https://www.aging.senate.gov/imo/media/doc/02_Tietz_9_18_13.pdf

The Center for HIV Law and Policy - Kentucky (2017). Accessed at: https://www.hivlawandpolicy.org/states/kentucky

Thomas, A., Menon, A., Boruff, J., Rodriguez, A. M., \& Ahmed, S. (2014). Applications of social constructivist learning theories in knowledge translation for healthcare professionals: a scoping review. Implementation Science, 9(1), 54.

Thomas, M. D., Blacksmith, J., \& Reno, J. (2000). Utilizing insider-outsider research teams in qualitative research. Qualitative health research, 10(6), 819-828.

Thompson, B. (1987). Foundations of behavioral research, -Kerlinger, FN.

Trotter, C., \& Baiawi S. (2015). Older prisoners: Challenges for inmates and prison management. Australian \& New Zealand Journal of Criminology, 48(2), 200-218.

UNAIDS (2012). UNAIDS report on stigma and discrimination.

United States Census Bureau (2018). 
U.S. Census (2016). National Population Projections.

U.S. Bureau of Justice Statistics (2015).

U.S. Department of Justice (2011).

U.S. Department of Justice (2015).

U.S. Department of Justice (2016). Aging of the Prison Population.

Wallace, S. A., McLellan-Lemal, E., Harris, M. J., Townsend, T. G., \& Miller, K. S. (2011). Why take an HIV test? Concerns, benefits, and strategies to promote HIV testing among low-income heterosexual African American young adults. Health Education \& Behavior, 38(5), 462-470.

Warren-Jeanpiere, L., Dillaway, H., Hamilton, P., Young, M., \& Goparaju, L. (2014). Taking it one day at a time: African American women aging with HIV and comorbidities. AIDS patient care and STDs, 28(7), 372-380.

Weitzer, R. (2017). Theorizing racial discord over policing before and after Ferguson. Justice quarterly, 34(7), 1129-1153.

Wertz, F., Charmaz, K., McMullen, L., Josselson, R., Anderson, R., \& McSpadden, E. (2011). Five ways of doing qualitative research. New York: Guilford Pres.

Westergaard, R. P., Spaulding, A. C., \& Flanigan, T. P. (2013). HIV among persons incarcerated in the US: a review of evolving concepts in testing, treatment and linkage to community care. Current opinion in infectious diseases, 26(1), 10.

Whiteley, L. B., Brown, L. K., Swenson, R. R., Romer, D., DiClemente, R. J., Salazar, L. F., ... \& Valois, R. F. (2011). African American adolescents and new media: Associations with HIV/STI risk behavior and psychosocial variables. Ethnicity \& disease, 21(2), 216.

Whiteside, Y. O., Cohen, S. M., Bradley, H., Skarbinski, J., Hall, H. I., \& Lansky, A. (2014). Progress along the continuum of HIV care among blacks with diagnosed HIV - United States, 2010. MMWR. Morbidity and mortality weekly report, 63(5), 85.

Williams, B. A., Stern, M. F., Mellow, J., Safer, M., \& Greifinger, R. B. (2012). Aging in correctional custody: setting a policy agenda for older prisoner health care. American journal of public health, 102(8), 1475-1481).

Wilper, A. P., Woolhandler, S., Boyd, J. W., Lasser, K. E., McCormick, D., Bor, D. H., \& Himmelstein, D. U. (2009). The Health and Health Care of US Prisoners: Results of a Nationwide Survey. American Journal of Public Health, 99(4), 666-672. http://doi.org/10.2105/AJPH.2008.144279

Wright, E. R., \& Carnes, N. (Eds.). (2016). Understanding the HIV/AIDS Epidemic in the United States: The Role of Syndemics in the Production of Health Disparities. Springer. 
Yarnell, S. C., Kirwin, P. D., Zonana, H. V. (2017). Geriatrics and the legal system. $J$ Am Acad Psychiatry Law 45(2), 208-217.

Zanoni, B. C., \& Mayer, K. H. (2014). The adolescent and young adult HIV cascade of care in the United States: exaggerated health disparities. AIDS patient care and STDs, 28(3), 128-135.

Zawitz, C., \& Parisot, J. (2013). The dangers of HIV stigma in corrections. Positively aware: the monthly journal of the Test Positive Aware Network, 26(6), 16-19. 


\section{APPENDICES}

\section{Appendix A}

\section{In-depth Interview Guide informed by the CHSCP}

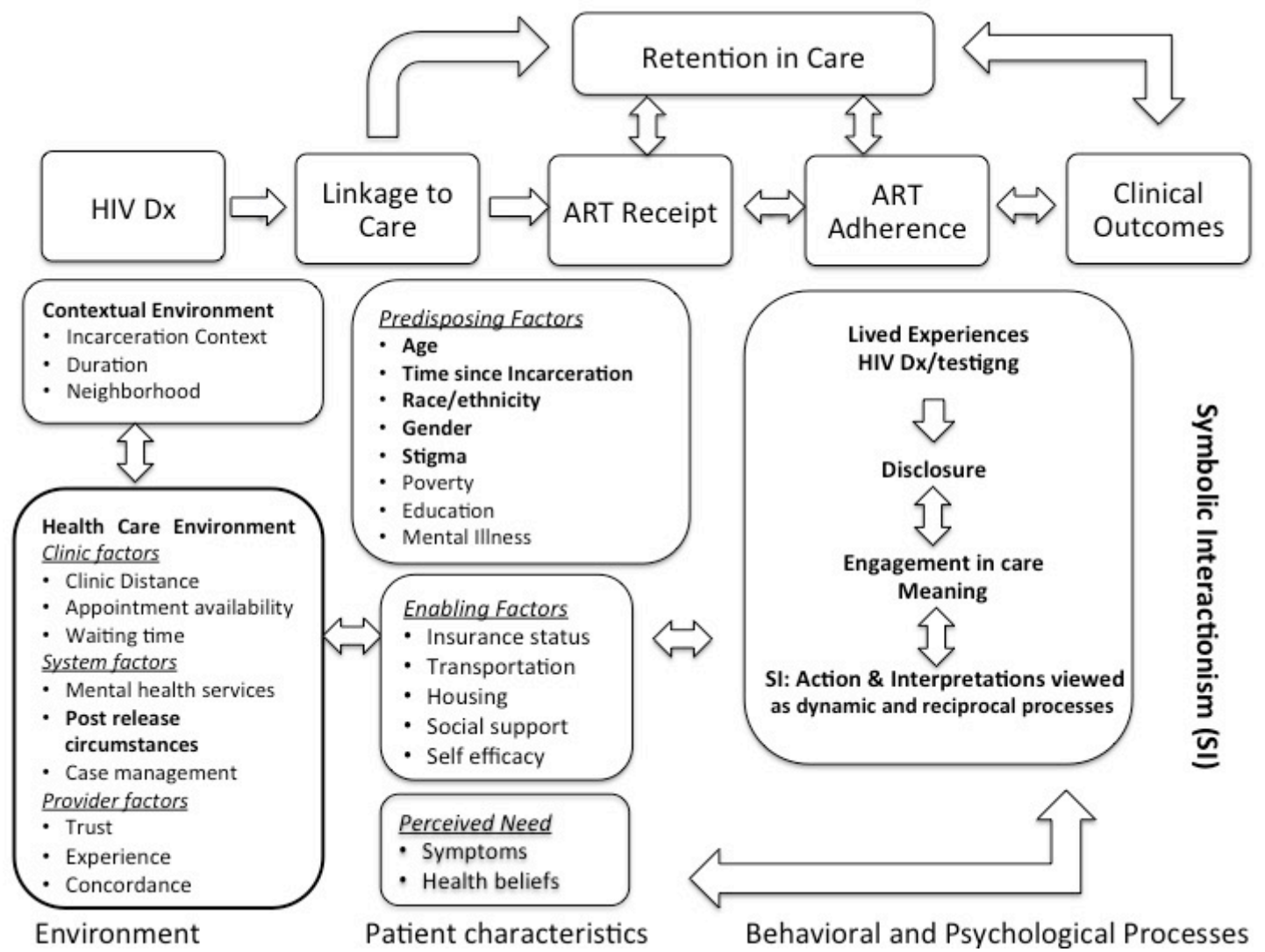

\section{Introduction}

\section{In-depth Interview}

1) Could you tell me about your experiences of living with HIV while you were incarcerated?

2a) Did you find out that you were HIV positive while you were incarcerated?

2b) If not, can you tell me about the first time that you saw a medical provider regarding your HIV diagnosis while you were incarcerated?

\section{Disclosure}

3) Can you tell me about a time you disclosed your HIV status to someone while incarcerated?

4) Who did you discloses your status to (other inmate, correctional officer, healthcare worker) and how was that experience?

\section{Stigma}

5) Can you tell me about a time when you felt discriminated against or mistreated because of HIV while you were incarcerated? 
6) Based on your experience is there a difference regarding HIV stigma when a person is incarcerated?

7) Do you think being older impacts ones' incarceration experience when living with HIV?

8) What do you think that society should know about being an older African American living having HIV and have an history of incarceration?

\section{HIV Care while Incarcerated}

9) How did your HIV care look like while you were incarcerated?

10) Can you tell me about the first time that you saw a medical provider regarding your HIV diagnosis while you were incarcerated?

\section{Engagement in Care}

11) What challenges did you face in your HIV care while incarcerated?

12) What or who has helped your overcome those challenges?

13) How did you experience health care workers and/or correctional officers responses to your needs regarding your HIV care and/or older age?

14) Can you tell me about a time when you felt very supported in living with HIV while your incarcerated? (e.g. by a healthcare worker or correctional officer)

15) Can you tell me about a time when you were turned off by a healthcare worker or correctional officer?

16) Have you ever not actively engaged in HIV care while incarcerated?

Probe: Can you tell me more about that time? What caused you to disengage?

17) Have you ever not taken your HIV medication while incarcerated?

Probe: Can you tell me more about that time? What caused you to not take your medication?

\section{Environmental}

18) Who were the important people in your life while you were incarcerated and how did they support you?

19) Have there been groups of people or organizations that have been particularly helpful to you living with HIV while incarcerated? Who were they? How did they help? (e.g. other HIV positive inmates)

20) Based on your experience how does an incarceration experience facilitate or hinder engagement in HIV care?

21) Has your incarceration experience impacted your access to HIV care? Did it make it easier or more difficult?

\section{Closing}

22) What advice would you give other older AAPLWHA about being incarcerated? If you were going to help other older African Americans trying to cope with a diagnosis of HIV while being incarcerated?

23) Are there other things you have learned about your incarceration experiences while living with HIV that you would like to share with me that we have not touched on? 


\section{Appendix B}

\section{Overall:}

\section{Focus Group Questions}

How does general life stress relate to engagement in HIV care?

How does HIV related stress impact engagement in HIV care?

We found that the majority of the people that we interviewed had multiple, co-morbid conditions (diabetes, heart disease), therefore, how does stress relate to engagement in health care?

The majority of the people that we interviewed had multiple, co-morbid conditions (diabetes, heart dz etc.). How do people navigate stress of multiple conditions? How does the stress from another health condition (co-morbidities, heart disease, diabetes) impact care/engagement in care?

How does stigma impact engagement in HIV care? Engagement in other kinds of health care?

Thinking of stress and stigma as influencers in your life, how does the combination of both factors impact your engagement in care?

\section{Qualitative follow up:}

We found that time was an important factor, and people's narrative reflected the past, present and future, which mirrors the process of aging with HIV. Therefore, we would like to ask you some questions about time. (Participants located their self-concepts in the past, present and future)

How has your experience with stress changed over time? (past, present, future) How has your experience with stigma changed over time? (past, present, future) How has engagement in care changed over time? (past, present, future) Can you tell us more about the unique experience of being and older, African American adult living with HIV? How do you imagine that this experience is similar or different than those who are living with HIV who are different than yourself?

What are some of the benefits of growing older with HIV? Other studies and our preliminary findings show that older adults seem to become more engaged in their care over time and see the benefit in more frequent contact with medical professionals. How do you relate to this?

\section{Quantitative follow up:}

-we found that stress is correlated with stigma and vs. versa

-disclosure concerns is correlated with stress

-negative self-image is correlated with stress

-the more negative one's self-image is, the less engaged in care one is

-personalized stigma is correlated with stress

-public attitudes are associated with stress

-the more stressed one is, the less engaged in care one is

-the more stigma experienced, the less engaged in care

Individuals who are currently using any type of substance are less likely to be engaged in care.

How has alcohol use among the group changed over time? Has this impacted engagement in care?

How has the use of other substances among the group changed over time? Has this impacted engagement in care?

-what are the differences between understandings of engagement in care vs. engagement with HCP? How does agency play in? Listening to doctors vs. following own plan for care. 


\section{Possible Incarceration Questions}

We found that a majority of our participants indicated an incarceration history.

What are your experiences with HIV (care/testing /disclosure) while being incarcerated?

Does incarceration impact engagement in care?

Have there been issues regarding disclosure/confidentiality among other inmates or prison guards?

Is there a difference in how you felt treated by medical personal versus prison employees? 


\section{Appendix C}

\section{Introduction}

\section{Original Study: In-depth Interview Guide}

1) Could you tell me about your experiences in living with HIV?

2) How did you find out that you were HIV positive?

3) Can you tell me about the first time that you saw a doctor regarding your HIV diagnosis?

\section{Stress}

4) Thinking about your life right now, what causes you stress?

5) What challenges do you face in your HIV care?

6) What or who has helped your overcome challenges in your HIV care?

\section{Stigma}

7) Can you tell me about a time when you felt discriminated against or mistreated because of HIV?

8) What do you think that society should know about being an older African American and having HIV?

9) How has your family responded to your diagnosis?

\section{Questions about HIV Clinic}

10) How did you find out about your HIV clinic?

11) What made you first come here?

12) What were your first impressions?

13) How have your opinions on your HIV clinic changed over time?

\section{Engagement in Care}

14) What makes you want to keep coming back to your clinic for care?

15) Can you tell me about a time when you felt very supported by a healthcare worker?

16) Can you tell me about a time when you were turned off by a healthcare worke

17) Have you ever missed an appointment with a Health Care Professional?

Probe: Can you tell me more about that time? What caused you to miss that appointment?

18) Have you ever not taken your HIV medication?

Probe: Can you tell me more about that time? What caused you to not take your medication?

\section{Environmental}

19) Can you tell me about the important people in your life and how they help you?

20) Have there been groups of people or organizations that have been particularly helpful to you living with HIV? Who are they? How did they help?

21) What is still missing from the healthcare system that would help people living with HIV?

22) What is lacking in terms of HIV care in Louisville? How can we make it better?

23) Are there parts of the environment such as where in Kentucky you live, housing or transportation that make accessing HIV care easier? How about more difficult?

24) Since the initiation of the Affordable Care Act "Obamacare" (1/1/2014) have you noticed any changes with your insurance?

\section{Closing}

25) What do you think you would tell others about living with HIV if you were going to 
help other older African Americans trying to cope with a diagnosis of HIV?

26) Are you currently participating in an HIV support group?

- If yes, then what is beneficial about this group?

- If no, what are the barriers to attending a group?

27) Are there other things you have learned about how to successfully access healthcare that you would like to share with me that we have not touched on? 


\section{Appendix D}

\section{IRB APPROVED DOCUMENTS FOR ORIGINAL STUDY}

UofL Institutional Review Boards
IRB NUMBER: 16.1274
IRB APPROVAL DATE: 07/24/2016
IRB EXPIRATION DATE: 04/21/2017

African American Older Adults Living with HIV: Exploring Stress, Stigma, and Engagement in HIV Care

INFORMED CONSENT AND RESEARCH AUTHORIZATION African American Older Adults Living with HIV: Exploring Stress, Stigma, and Engagement in
HIV Care

Investigator(s) name, Degree, University Department, \& address:

Timothy N Crawford, PhD Lesley Harris, PhD, MSW

University of Louisville, University of Louisville, Kent

School of Nursing School of Social Work

555 S. Floyd St. 109 Patterson Hall

Louisville, KY $40202 \quad$ Louisville, KY 40292

Jelani Kerr, PhD, MSPH

University of Louisville,

School of Public Health and

Information Sciences

485 E. Gray St. \#208

Louisville, KY 40202

Site(s) where study is to be conducted:

KY Care Coordination Clinic

550 HIV Clinic

$501 \mathrm{E}$. Broadway

Suite 120

Louisville, KY 40202

500 South Preston St.

Room 208

Louisville, KY 40202

Phone number for subjects to call for questions:

Timothy Crawford (502) 852.8510

Introduction and Background Information

You are invited to take part in a research study. The study is being conducted under the directions of Timothy N Crawford, PhD, MPH, faculty member at the University of Louisville School of Nursing, Lesley Harris, PhD, MSW, faculty member at the University of Louisville Kent School of Social Work and Jelani Kerr, PhD, MSPH, faculty member at the University of Louisville School of Public Health and Information Sciences. The study will take place primarily at the 550 HIV clinic. Approximately 50 older adults (greater than or equal to 50 years of age) will be invited to participate in this research.

\section{Purpose}

The purpose of this study is to examine the effects of stigma and perceived stress on engagement in care for older African Americans living with HIV.

\section{Procedures}

You will be asked to sign a consent form giving us your permission to participate in the study. After you sign the consent form, you will be asked to provide us with information at potentially 4 different time points. At the first interview, you will be asked to complete a survey that will ask questions about you (e.g., age, gender, race, education, etc.), your clinical history, your use of health care services, and level of stress and stigma related to living with HIV. After the first interview, if you are eligible, you will be contacted to complete a second interview which will examine stress, stigma, and use of health

Version Date: 4/7/2016 Page 1 of 9 
care services in more detail. This interview will give you the opportunity to express your experiences living with HIV and how stress and stigma have had an impact on your use of health care services. Next, you may be contacted to provide a follow-up interview to discuss in more detail your experiences. Finally, you may be contacted to participate in the final interview of the study, which will be a focus group. In the last two interviews of the study, the interviews will be recorded by a member of the research team. Each interview will take approximately 30 to 60 minutes of your time. All data will be collected by a member of our research team. The entire study will take about 8 months to complete. You may decline answering any of the questions at any time if the questions make you uncomfortable. You may also choose to not allow the research team to collect any data that makes you feel uncomfortable.

\title{
Potential Risks
}

There are no major foreseeable risks to participate in the study. A participant may have some possible discomfort in answering personal questions while participating in the study. In addition, there is the possible risk of loss of confidentiality. In order to prevent this from happening, all identifying information will de-identified. We will do everything to keep their data secure.

\section{Benefits}

The information that is collected during this study, may not benefit you directly. However, the information learned from this study may be helpful to others.

\section{Alternatives}

Instead of taking part in this study, you could choose to not participate in this study.

\section{Research Related Injury}

If you are injured by being in this research study, the study doctor will arrange for you to get medical treatment. The study site, or your study doctor has not set aside money to pay for treatment of any injury. You and your insurance will be billed for the treatment of these injuries. Before you agree to take part in this research study, you should find out whether your insurance will cover an injury in this kind of research. You should talk to the study doctor or staff about this. If you are injured, there is no money set aside for lost wages, discomfort, disability, etc. You do not give up your legal rights by signing this form. If you think you have a research related injury, please call your study doctor (Timothy Crawford, 859-967-8859).

\section{Compensation}

At the first interview of the study, you will be compensated with $\$ 15$ gift card. After the second interview of the study, you will be compensated with a $\$ 20$ gift card. After the follow-up interview, you will be compensated with a $\$ 20$ gift card. At the third interview of the study, you will be compensated with a $\$ 25$ gift card.

You will be paid by Visa gift card for your time, inconvenience, or expenses while you are in this study. A total of $\$ 80$ will be paid for your time. A total of $\$ 15$ will be distributed at the first visit, $\$ 20$ will

\author{
Version Date: 4/7/2016 Page 2 of 9
}


be distributed at the second visit, $\$ 20$ will be distributed at the follow-up visit, and $\$ 25$ will be distributed at the final visit. Because you will be paid to be in this study the University of Louisville may collect your name, address, social security number, and keep records of how much you are paid. You may or may not be sent a Form 1099 by the University. This will only happen if you are paid $\$ 600$ or more in one year by the University. This will not include payments you may receive as reimbursement, for example mileage reimbursement. We are required by the Internal Revenue Service to collect this information and you may need to report the payment as income on your taxes. You can still be in the study even if you don't want to be paid.

\section{Costs}

There will be no costs to you for participating in this research. You will not be billed for the tests, medications and procedures that are done for this research study. The charges for these items will be paid for by the research.

\section{HIPAA Research Authorization}

The Health Insurance Portability and Accountability Act of 1996 (HIPAA) provides federal safeguards for your protected health information $(\mathrm{PHI})$. Examples of $\mathrm{PHI}$ are your name, address, and birth date together with your health information. PHI may also include your medical history, results of health exams and lab tests, drugs taken and results of this research study. Your PHI may not be used or shared without your agreement, unless it meets one of the HIPAA exceptions.

State and federal privacy laws protect your health information. In most cases, health information that identifies you can be used or shared by the research team only if you give your permission by signing this form.

If you sign this form your health information will be used and shared to answer the research questions described above and to make sure that the research was done correctly. The time period when information can be used or shared ends when all activities related to this study are completed.

Your access to your health information will not be limited during this study.

You do not have to sign this form. If you do not sign this form you may not participate in the study and health information that identifies you will not be shared with the research team.

\section{Site(s) where health information about you will be used or shared for this research:}

In our research, the research team will look at and may share information about you and your health. Federal law requires that health care providers and researchers protect the privacy and security of health information that identifies you. We may ask for your health information from the following:

\section{Affiliated Sites:}

University of Louisville 


\section{University of Louisville Research Foundation (ULRF) Clinical Sites:}

HIV Care Coordination Clinic

550 HIV Clinic

\section{Protected health information (PHI) that will be used or shared for research}

Name

Date of Birth

Sex

Ethnicity

Phone Number

Email Address

Diagnoses and Dates

\section{Revocation of Research Authorization}

You may cancel the permission you have given to use and share your protected health information at any time. This means you can tell us to stop using and sharing your protected health information. If you cancel your permission:

- We will stop collecting information about you.

- You may not withdraw information that we had before you told us to stop.

○ We may already have used it or shared it.

- We may need it to complete the research.

- Staff may ask your permission to follow-up with you if there is a medical reason to do so.

To cancel your permission, you will be requested to complete a written "Revocation of Research Authorization" form located at the end of this document. You may also obtain a copy from your study doctor, designated personnel or from the Human Subjects Protections Program Office website (http://louisville.edu/research/humansubjects/links-to-forms).

\section{Information Available on ClinicalTrials.gov}

A description of this clinical trial will be available on http://www.ClinicalTrials.gov, as required by U.S. Law. This website will not include information that can identify you. At most, the website will include a summary of the results. You can search this website at any time.

\section{Confidentiality}

Total privacy cannot be guaranteed. We will protect your privacy to the extent permitted by law. If the results from this study are published, your name will not be made public. Once your information leaves our institution, we cannot promise that others will keep it private.

Your information may be shared with the following:

Version Date: 4/7/2016 Page 4 of 9 
- The University of Louisville and others hired by the sponsor to oversee the research

- The University of Louisville Institutional Review Board, Human Subjects Protection Program Office, Privacy Office and others involved in research administration at the University

- The local research team

- People who are responsible for research and HIPAA oversight at the institutions where the research is conducted

- People responsible for billing, sending and receiving payments related to your participation in the study

- Government agencies, such as:

- Office for Human Research Protections

- Office of Civil Rights

\section{Security}

Your information will be kept private by being place in a secured locked file cabinet or on a password protected computer file where only the Principal investigators will have access.

\section{Voluntary Participation}

Taking part in this study is completely voluntary. You may choose not to take part at all. If you decide not to be in this study, you won't be penalized or lose any benefits for which you qualify. If you decide to be in this study, you may change your mind and stop taking part at any time. If you decide to stop taking part, you won't be penalized or lose any benefits for which you qualify. You will be told about any new information learned during the study that could affect your decision to continue in the study.

\section{Termination}

Your study doctor or the study sponsor has the right to stop this study at any point. Your study doctor may take you out of this study with or without your okay. Reasons why this may occur include: Not showing up for the next scheduled interview.

\section{Participation in Other Research Studies}

You may take part in this study if you are currently in another research study. It is important to let your doctor know if you are in another research study.

\section{Contact Persons}

If you have any questions, concerns, or complaints about the research study, please contact Timothy Crawford (502) 852.8510

\section{Research Subject's Rights}

Version Date: 4/7/2016 Page 5 of 9 
If you have any questions about your rights as a research subject, you may call the Human Subjects Protection Program Office at (502) 852-5188. You may discuss any questions about your rights as a research subject, in private, with a member of the Institutional Review Board (IRB). You may also call this number if you have other questions about the research, and you cannot reach the study doctor, or want to talk to someone else. The IRB is an independent committee made up of people from the University community, staff of the institutions, as well as people from the community not connected with these institutions. The IRB has approved the participation of human subjects in this research study.

\section{Concerns and Complaints}

If you have concerns or complaints about the research or research staff and you do not wish to give your name, you may call the toll free number 1-877-852-1167. This is a 24 hour hot line answered by people who do not work at the University of Louisville.

\section{Acknowledgment and Signatures}

This informed consent document is not a contract. This document tells you what will happen during the study if you choose to take part. Your signature indicates that this study has been explained to you, that your questions have been answered, and that you agree to take part in the study. You are not giving up any legal rights to which you are entitled by signing this informed consent document. You will be given a copy of this consent form to keep for your records.

Subject Name (Please Print)

Printed Name of Legal Representative (if applicable)

Signature of Legal Representative
Date Signed

Date Signed

Relationship of Legal Representative to Subject

Printed Name of Person Explaining Consent Form

Signature of Person Explaining Date Signed Consent Form (if other than the Investigator)

Printed Name of Investigator

Signature of Investigator

Date Signed

List of Investigators:

Timothy N Crawford, PhD, MPH

Phone Numbers:

Lesley Harris, PhD, MSW

(502) 852.8510

(502) 852.8316

Version Date: 4/7/2016 Page 6 of 9 
ofL Institutional Review Board

RB NUMBER: 16.1274

IRB APPROVAL DATE: 07/24/2016

IRB EXPIRATION DATE: 04/21/2017

African American Older Adults Living with HIV: Exploring Stress, Stigma, and Engagement in HIV Care

Jelani Kerr, PhD, MSPH

(502) 852.3291

Version Date: 4/7/2016 Page 7 of 9 
African American Older Adults Living with HIV: Exploring Stress, Stigma, and Engagement in HIV Care

REVOCATION OF AUTHORIZATION FOR USE AND DISCLOSURE OF YOUR HEALTH INFORMATION FOR RESEARCH

\begin{tabular}{l}
\hline PI Address: University of Louisville School of \\
Nursing \\
555 South Floyd St. \\
Louiwville, KY 40202 \\
Phone: (502)852.8510 \\
\hline To Whom It May Concern:
\end{tabular}

Return To: Institutional Review Board MedCenter One, Suite 200

OR

501 E. Broadway

Louisville, KY 40202

I would like to discontinue my participation in the research study noted above. I understand that health information already collected will continue to be used as discussed in the Authorization I signed when joining the study.

Your options are (choose one):

$\square$ Withdraw from Study \& Discontinue Authorization:

Discontinue my authorization for the future use and disclosure of protected health information. In some instances, the research team may need to use your information even after you discontinue your authorization, for example, to notify you or government agencies of any health or safety concerns that were identified as part of your study participation.

\section{$\square$ Withdraw from Study, but Continue Authorization:}

Allow the research team to continue collecting information from me and my personal health information. This would be done only as needed to support the goals of the study and would not be used for purposes other than those already described in the research authorization.

Printed Name and Signature of Subject Date Signed

$\overline{\text { Signature of Subject's Legal Representative (if subject is unable to sign) }}$ Date Signed

Printed Name of Subject's Legal Representative Birthdate of Subject

Relationship of Legal Representative to Subject

Optional:

I am ending my participation in this study because: 
ofL Institutional Review Board

RB NUMBER: 16.1274

IRB APPROVAL DATE: 07/24/2016

IRB EXPIRATION DATE: 04/21/2017

African American Older Adults Living with HIV: Exploring Stress, Stigma, and Engagement in HIV Care

Version Date: 4/7/2016 Page 9 of 9 


\section{CURRICULUM VITAE}

Verena Schmidt

verena.schmidt@louisville.edu

4564 Clark Station Road

Finchville, KY 40022

502-296-7617

\section{Education}

PhD

University of Louisville,

Expected

Kent School of Social Work

MSSW

May 2011

University of Louisville

Kent School of Social Work

BA Katholische Stiftungsfachhochschule Munich

January 2010

University of Applied Sciences

Department: Social Pedagogy and Social Work

Concentration: Family Studies and Mental Health

\section{Professional Experience}

Instructor

2016-2020

Kent School of Social Work

Advanced Research Practice I \& II

Research Methodology and Design

Research Assistant

2016-2018

University of Louisville "African American Older Adults Living with HIV: Exploring Stress, Stigma and Engagement in HIV Care." Funded by: Executive Vice President for Research and Innovation's Internal Research Grant Program

Member of a research team that conducted a pilot mixed-methods study on stress, stigma and engagement in care among older African American Adults living with HIV/AIDS throughout Louisville, Kentucky.

Research Assistant

2016-2018

University of Louisville "Examining Stress, Stigma and Engagement in HIV Care

Utilization among African American Elders." Funded by: Cooperative Consortium for Transdisciplinary Social Justice Research Grant

Women's Center, University of Louisville

2010-2011

MSSW Field Placement 
- Connected international students to resources on campus

- Worked on several projects including a workshop facilitating the transition to the U.S. for international students

"Carpe Diem" Munich, Germany

2009-2010

Social Work Caseworker

Description: Case Management for older adults suffering from dementia, Alzheimer's and comorbid mental illness

- Case management

- Co-lead family support group helped families better understand a family member's illness and worked with them to develop coping skills

Mindzone "Sauber drauf!" Munich, Germany

$2007-2010$

Mobile drug counseling and Pprevention for youths and young adults

Description: Social Work Intern

Frauentherapiezentrum Munich, Germany

$2007-2010$

Social Work Intern and Caseworker

Description: Intensive outpatient treatment center for woman struggling with alcohol abuse and eating disorders

Kindergarten "St. Gabriel”, Raisting, Germany

2004-2006

Intern

A daycare/child development center in rural Bavaria, Germany

\section{Research Experience}

\section{Principal Investigator}

2016-2020

Dissertation Research: "Incarceration Experiences of older African American adults: A Constructivist grounded theory study." I designed and administered a qualitative study on the incarceration experiences of older African American adults living with HIV/AIDS.

Research Assistant 2016-2018

University of Louisville "Examining Stress, Stigma and Engagement in HIV Care Utilization among African American Elders." Funded by: Cooperative Consortium for Transdisciplinary Social Justice Research Grant

Member of a research team that utilized monologue development through reengaging previous participants in selection and editing of transcript passages for dramatization. Stigma and engagement in care among older African American adults living with HIV/AIDS throughout Louisville, Kentucky.

Research Assistant 2016-2018

University of Louisville "African American Older Adults Living with HIV: Exploring Stress, Stigma and Engagement in HIV Care." Funded by: Executive Vice President for Research and Innovation's Internal Research Grant Program

Member of a research team that conducted a pilot mixed-methods study on stress, stigma and engagement in care among older African American adults living with HIV/AIDS throughout Louisville, Kentucky. 
HIV and Health Care Support Centre (Hai Phong, Vietnam) Community-based support to elderly caregivers of vulnerable children in Hai Phong, Vietnam. Funded by: Korean International Cooperation Agency (KOICA)

Projects included exploring "Health Care Access Among Grandparent-Headed Households Impacted by HIV/AIDS in Vietnam and investigating the effect of Empathy Clubs" and "Exploring Household Food Insecurity in GrandparentHeaded Households in Hai Phong, Vietnam."

Graduate Research Assistant,

2014 - June 2016

University of Louisville

Kent School of Social Work

Advisor: Lesley Harris, Ph.D.

I was extensively trained in qualitative methodology and assisted on several research projects, such as the data analysis health seeking behaviors among homeless youth in Ho Chi Minh City, Vietnam.

Graduate Research Assistant,

2013-2014

University of Louisville

Kent School of Social Work

Advisor: Thomas Lawson, Ph.D.

Assisted in the development of a German-English-Hungarian dictionary for social work and guest lectured about social work education in Germany in the MSSW program.

Graduate Research Assistant,

$2011-2012$

University of Louisville

Kent School of Social Work

Advisor: Sharon Bowland, Ph.D.

Conducted literature reviews and trained in qualitative research methods.

\section{Teaching Experience}

\section{Instructor}

University of Louisville, Kent School of Social Work

MSSW Program

Fall 2019/ Spring 2020

668-53 Advanced Research Practice I and II online

\section{Instructor}

University of Louisville, Kent School of Social Work

Fall 2018/ Spring 2019

MSSW Program

668-53 Advanced Research Practice I and II online

\section{Instructor}

University of Louisville, Kent School of Social Work MSSW Program

SW-668-53 Advanced Research Practice I and online

SW-669-58 Advanced Research Practice I and II online 


\section{Instructor}

University of Louisville, Kent School of Social Work

Fall 2016 / Spring 2017

MSSW Program

SW 668 - 53 Advanced Research Practice I and II online

SW 669 - 04 Advanced Research Practice I and II online

\section{Instructor}

University of Louisville, Kent School of Social Work

Summer 2016

MSSW Program:

SW 626 - 01 Research Methodology and Design

Co- Instructor

University of Louisville, Kent School of Social Work

Spring 2016

BSW \& MSSW Program:

Social Work Service Learning in Botswana

Teaching Assistant

Summer 2015

University of Louisville, Kent School of Social Work

MSSW Program: Diversity, Oppression \& Social Justice Practice

\section{Publications}

Boggiano, V., Harris, L.M., Schmidt, V., Nguyen, T. \& Barry, M. (2017). "Protecting, Balancing and Confronting: Health-Seeking Among Homeless Youth in Ho Chi Minh City, Vietnam." International Journal of Child, Youth and Family Studies.

Harris, L.M., Crawford, T.N., Kerr, J., Thomas, T.A. \& Schmidt, V. (accepted) “African American Older Adults living with HIV: Exploring Stress, Stigma and Engagement in HIV Care." Journal of Health Care for the Poor and Underserved.

Harris, L. M., Wilfong, J., Schmidt, V., \& Thang, N. (2017). "Effects of Empathy Clubs on Health Care among HIV-affected Grandparent-headed Households in Vietnam." Innovation in Aging, 1(Suppl 1), 531-532. doi:10.1093/geroni/igx004.1880

\section{Publications in Progress}

Schmidt V., Harris L., Thomas T., Kerr, J. and Crawford, T. (2017). "Incarceration Experiences of Older African American Adults living with HIV: How Stigma and HIV Testing Impact Engagement in Care."

Harris, L.M., Schmidt, V., Wilfong, J. \& Thang, N.D. "Exploring Household Food Insecurity in Grandparent-Headed Households Who Have Been Impacted By HIV/AIDS in Vietnam."

\section{Peer reviewed Conference Presentations}

Schmidt, V., Alvey Thomas, T., Harris, L.M., Kerr, J. (2018). Transforming Qualitative Data into HIV Monologues. International Congress of Qualitative Inquiry. Champaign, IL. May, 19, 2018. 
Schmidt, V. (2018). Incarceration Experiences of Older African American Adults living with HIV - A Constructivist Grounded Theory Study. International Congress of Qualitative Inquiry. Champaign, IL. May, 19, 2018.

Harris, L., Crawford, T., Kerr, J., Alvey T. and Schmidt, V. (2017). "African American Older Adults Living with HIV: Exploring Stress, Stigma and Engagement in HIV Care." American Public Health Association (APHA) Annual Meeting and Expo. Atlanta, Georgia November 4-8 ${ }^{\text {th }}, 2017$.

Schmidt V., Harris L., Thomas T., Kerr, J. and Crawford, T. (2017). "Incarceration Experiences of Older African American Adults living with HIV: How Stigma and HIV Testing Impact Engagement in Care." American Public Health Association (APHA) Annual Meeting and Expo. Atlanta, Georgia November 4-8 ${ }^{\text {th }}, 2017$.

Harris, L.M., Wilfong, J., Schmidt, V. \& Thang, N.D. "Effects on Empathy Clubs Health Care among HIV affected Grandparent-Headed Households in Vietnam." 23rd Francisco world congress on geriatrics and gerontology. San Francisco, California, July 23-27 th

Schmidt V., Harris L., Thomas T. (2017). "HIV + Incarceration: A Social Justice Issue Impacting Older African American Adults." $29^{\text {th }}$ Annual Conference on Social Work and HIV/AIDS. Atlanta, Georgia, May 25-28 2017

Boggiano, V., Harris, L.M., Schmidt, V. \& Barry, M. (2015). Homeless Youth in Vietnam: A Qualitative Inquiry. Stanford Global Health Research Convening. Stanford, CA. January, 9, 2015.

\section{Workshop Presentations}

Harris, L., Crawford, T., Kerr, J., Schmidt, V., Alvey T., and Nanette Bowman (2017). :Older African American Adults Living with HIV: Exploring Stress, Stigma, and Engagement in Care: Recommendations for Service Providers."

Schmidt, V., Harris, L., Crawford, T., Kerr, J., Alvey T. (2017). "Incarceration Experiences of Older African American Adults Living with HIV: Implications for Practice when Working with Formerly Incarcerated Individuals." International Congress for Qualitative Inquiry, Champaign, Illinois, May, 2017 